\title{
THE GEOMETRIES OF 3-MANIFOLDS
}

\author{
PETER SCOTT
}

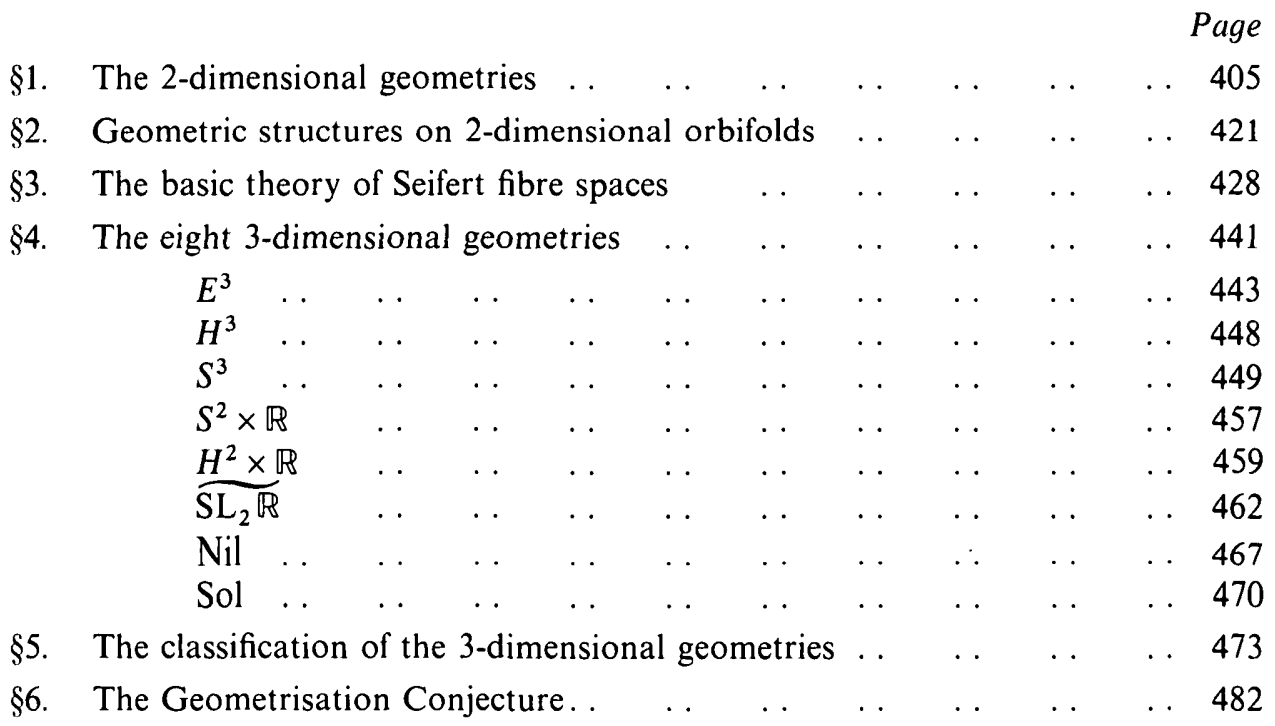

\section{Introduction}

The theory of 3-manifolds has been revolutionised in the last few years by work of Thurston [66-70]. He has shown that geometry has an important role to play in the theory in addition to the use of purely topological methods. The basic aim of this article is to discuss the various geometries which arise and explain their significance for the theory of 3-manifolds. The idea is that many 3-manifolds admit 'nice' metrics which give one new insight into properties of the manifolds. For the purposes of this article, the nicest metrics are those of constant curvature. An observer in a manifold with a constant curvature metric will see the same picture wherever he stands and in whichever direction he looks. Such manifolds have special topological properties. However, we will also need to consider nice metrics which are not of constant curvature. In this article, I will explain what is meant by a 'nice' metric and describe their classification in dimension three which is due to Thurston. Then I will discuss some of the 3-manifolds which admit these nice metrics and the relationship between their geometric and topological properties. In this introduction all manifolds and metrics will be assumed to be smooth so that the objects of interest are all Riemannian manifolds.

It has been known since the nineteenth century that in dimension two there is a very close relationship between geometry and topology. I will start by describing some basic facts about closed surfaces. I will discuss these in more detail in $\$ 1$. Each 
closed surface admits a metric of constant curvature and these metrics can be described very simply. There are three cases depending on the Euler number of the surface.

There are only two closed surfaces with positive Euler number, namely the 2-sphere $S^{2}$ and the real projective plane $P^{2}$. The standard metric on Euclidean 3-space $\mathbb{R}^{3}$ induces a metric on the unit sphere $S^{2}$ which has constant positive curvature, equal to 1 . One way of thinking of this metric on $S^{2}$ is first to realise that each smooth path on $S^{2}$ has a length, namely its length as a path in $E^{3}$. (I shall consistently use $E^{n}$ to denote $\mathbb{R}^{n}$ with the standard metric.) One can then recover the Riemannian metric from this information. One has a metric on $S^{2}$ given by taking the distance apart of two points to be the infimum of the lengths of all paths between them. The projective plane $P^{2}$ is double covered by $S^{2}$ so the projection map $S^{2} \rightarrow P^{2}$ has the path lifting property. This allows one to define a metric on $P^{2}$ by defining the length of any path in $P^{2}$ to equal the length of a lift in $S^{2}$. Now a path in $P^{2}$ will have two lifts to $S^{2}$, and it is obviously essential for this definition that both lifts have the same length. This holds, because the non-trivial covering transformation of $S^{2}$ is an isometry, namely the antipodal map of $S^{2}$. Thus $P^{2}$ inherits from $S^{2}$ a metric such that the projection $S^{2} \rightarrow P^{2}$ is locally an isometry. In particular, this metric on $P^{2}$ has constant positive curvature, equal to 1 . Note that essentially the same argument applies whenever a manifold $M$ of constant curvature is a regular covering of a manifold $N$ and the covering group is a group of isometries of $M$. The conclusion is that $N$ inherits a metric of constant curvature.

There are only two closed surfaces with zero Euler number, namely the torus $T$ and Klein bottle $K$ and they both admit a metric of constant, and zero, curvature. Such metrics are called flat metrics. To obtain these metrics, start with the Euclidean plane $E^{2}$ with the standard metric. This is the archetypal flat manifold. If $G$ is a group of isometries of $E^{2}$ generated by two translations in independent directions, then the quotient of $E^{2}$ by $G$ is homeomorphic to the torus $T$ and inherits a flat metric. One can obtain a flat metric on the Klein bottle $K$ by taking the quotient of $E^{2}$ by a group $G$, where $G$ is generated by a translation and a glide reflexion in independent directions.

All the other closed surfaces have negative Euler number and admit metrics of constant negative curvature, equal to -1 . Each such surface can be obtained as a quotient of the hyperbolic plane $H^{2}$ by a group of isometries $\Gamma$ such that the projection $H^{2} \rightarrow H^{2} \mid \Gamma$ is a covering map.

The geometry of straight lines in $E^{2}$ is, of course, classical Euclidean geometry and the geometries of geodesics on the sphere $S^{2}$ and the hyperbolic plane $H^{2}$ are the less classical non-Euclidean geometries. If $X$ denotes one of $S^{2}, E^{2}$ or $H^{2}$ and if $F$ is a closed surface which can be expressed as a quotient of $X$ by a group $\Gamma$ of isometries of $X$ such that the projection $X \rightarrow X \mid \Gamma$ is a covering map, we will say, following Thurston, that $F$ possesses a geometric structure modelled on $X$. We have just seen that each closed surface admits a geometric structure. It is not hard to show that a closed surface $F$ admits geometric structures modelled on only one of $S^{2}, E^{2}$ and $H^{2}$.

It seems natural to ask whether the same kind of division of 3-manifolds into different geometric types occurs, but this question has only recently been asked by Thurston. One must first decide which are the types of geometry in which one should be interested. There are three obvious geometries which correspond directly to the two-dimensional ones, namely the constant curvature geometries $S^{3}, E^{3}$ and $H^{3}$, but it is easy to find closed 3-manifolds which cannot possess a geometric structure 
modelled on any of these. For example, $S^{2} \times S^{1}$ has universal covering $S^{2} \times \mathbb{R}$, which is not homeomorphic to $S^{3}$ or $\mathbb{B}^{3}$. (Note that $E^{3}$ and $H^{3}$ are each homeomorphic to $\mathbb{R}^{3}$.) However, $S^{2} \times \mathbb{R}$ and $S^{2} \times S^{1}$ each possesses a very natural metric which is simply the product of the standard metrics. This metric on $S^{2} \times \mathbb{R}$ differs from those on $S^{3}, E^{3}$ and $H^{3}$ by being anisotropic. If you stand at a point in $S^{2} \times \mathbb{R}$, the manifold will look different in different directions. However, the metric is still homogeneous, by which I mean that the manifold looks the same at different points. A more precise way of describing these properties of the metric is to say that the isometry group of $S^{2} \times \mathbb{R}$ acts transitively and that the stabiliser of a point is not $O(3)$, as in the constant curvature case. In fact, the isometry group of $S^{2} \times \mathbb{R}$ is the direct product of the isometry group of $S^{2}$ and of $\mathbb{R}$, and so the stabiliser of a point is isomorphic to $\mathrm{O}(2) \times \mathbb{Z}_{2}$.

This is a very natural example and leads to the following generalisation of the idea of a geometric structure. A metric on a manifold $M$ is said to be locally homogeneous if given $x$ and $y$ in $M$, there are neighbourhoods $U$ and $V$ of $x$ and $y$ and an isometry $(U, x) \rightarrow(V, y)$. We will say that $M$ admits a geometric structure if $M$ admits a complete, locally homogeneous metric. Now any covering space $\bar{M}$ of $M$ inherits a natural metric such that the projection $\tilde{M} \rightarrow M$ is locally an isometry. Hence if $M$ admits a geometric structure, the universal covering space $X$ of $M$ inherits a complete, locally homogeneous metric. It is a theorem of Singer [63] that such a metric on a simply connected manifold must be homogeneous, i.e. the isometry group of $X$ must act transitively. Thus we can regard $X$ together with its isometry group as a geometry in the sense of Klein, and we can sensibly say that $M$ admits a geometric structure modelled on $X$. Thurston has classified the 3-dimensional geometries and there are eight of them. See $\$ 4$ and $\$ 5$.

It is easy to see from the classification of these geometries that many closed 3-manifolds do not possess a geometric structure. For example, no connected sum of closed manifolds, except for $P^{3} \# P^{3}$, can admit a geometric structure. However, Thurston has conjectured (the Geometrisation Conjecture) that any compact, orientable 3-manifold $M$ can be cut by disjoint embedded 2-spheres and tori into pieces which, after glueing 3-balls to all boundary spheres, admit geometric structures. (Note that we say that a manifold with boundary has a geometric structure when the interior has such a structure.) This conjecture asserts that the situation for 3-manifolds is similar to that for surfaces. All the evidence points to this being the case, but it is a long way from being proved. For example, the Poincare Conjecture is a very special case of Thurston's conjecture. However, Thurston's most important result in this area asserts that his conjecture is correct if $M$ is a Haken manifold. See $\$ 6$ for a discussion of this.

Of the eight 3-dimensional geometries, seven are very well understood. In particular, one can classify all the 3-manifolds which possess a geometric structure of one of these seven types. The last and most difficult geometry is that of hyperbolic space $H^{3}$ and this is the area where Thurston has done most of his work. I intend to discuss mainly the seven easy geometries in this article as these have received little attention, apart from $S^{3}$ and $E^{3}$. These seven geometries correspond to the two geometries $S^{2}$ and $E^{2}$ in dimension two, in the sense that fairly few 3-manifolds can possess any of these geometric structures. However, all but two of the seven geometries give rise to infinitely many 3 -manifolds which is certainly different from the situation in dimension two. It turns out that the closed manifolds belonging to six of these geometries are precisely the Seifert fibre spaces, a class of 3-manifolds which 
has been extensively studied since Seifert first defined and classified them [59]. Interestingly, Seifert fibre spaces also appear when one considers non-positive definite metrics. See [29].

The neatest definition of a compact Seifert fibre space is simply a 3-manifold which can be foliated by circles, though it is quite difficult to prove that this is equivalent to Seifert's definition. Also Seifert's definition is more immediately useful. For some of the geometries, it is not surprising that any compact 3-manifold possessing a geometric structure modelled on them must be a Seifert fibre space. For example, $S^{2} \times \mathbb{R}$ and $H^{2} \times \mathbb{R}$ are foliated by the lines $\{x\} \times \mathbb{R}, x$ in $S^{2}$ or $H^{2}$, and the lines of these foliations are permuted by the isometries of $S^{2} \times \mathbb{R}$ and $H^{2} \times \mathbb{R}$. It follows that any 3-manifold with a geometric structure modelled on $S^{2} \times \mathbb{R}$ or $H^{2} \times \mathbb{R}$ is foliated by lines or circles. If the manifold is compact, it is usually true that the foliation is by circles. For some of the other geometries, it is much less obvious that they should give rise to Seifert fibre spaces. For example, it is known that any closed 3-manifold $M$ with a geometric structure modelled on $E^{3}$ (i.e. a flat manifold) must be a Seifert fibre space. The foliation of $M$ by circles gives rise to a $\pi_{1}(M)$-invariant foliation of $E^{3}$ by parallel straight lines, so that $\pi_{1}(M)$ must leave invariant a direction in $E^{3}$. But groups acting non-freely on $E^{3}$ certainly need not leave any direction invariant. For example, the symmetry group of a cube acts on $E^{3}$ fixing the origin and plainly leaves no direction invariant.

I will now describe the contents of this article in greater detail. In $§ 1$, I discuss the three plane geometries $S^{2}, E^{2}$ and $H^{2}$ giving an elementary introduction to the hyperbolic plane. I discuss discrete isometry groups of $S^{2}, E^{2}$ and $H^{2}$ and present the central results in this area. This very naturally leads to the second section, on orbifolds, which is based on Thurston's notes [66]. This term is due to Thurston and is used in nice cases to describe the quotient of a manifold by a group which need not act freely. This is called a $V$-manifold by some authors. As stated previously, each closed surface admits a geometric structure. The situation for orbifolds is similar in that almost every 2-dimensional orbifold admits a geometric structure.

In $\S 3$, I develop the basic theory of Seifert fibre spaces. A Seifert fibre space can be defined in various ways, but the most useful way of thinking of a Seifert fibre space is as a kind of bundle over a 2-dimensional orbifold with fibre the circle, and I take this viewpoint throughout. This allows a very geometric approach. Another new feature of my presentation is that I consider Seifert fibre spaces in which fibres can be orientation reversing loops. This generalises Seifert's original definition, but there are good reasons for regarding this new definition as being the correct one. With the new definition, a compact 3-manifold is a Seifert fibre space if and only if it is foliated by circles, as already stated. I hope that this section will be of interest to the experts as well as to those learning about Seifert fibre spaces for the first time. These more general spaces were considered by Orlik and Raymond [47] and Fintushel [16], but their approach was somewhat different from my approach in this article.

In $\$ 4$, I describe each of the eight 3-dimensional geometries and their basic properties. I give a complete description of the closed 3-manifolds which admit a geometric structure apart from those admitting a hyperbolic structure.

In $\S 5$, I sketch Thurston's proof that the eight geometries discussed in $\$ 4$ are the only geometries in which one need be interested. Then I use the properties of the geometries discussed in $\S 4$, to show that if a closed 3-manifold admits a geometric structure, then the geometry involved is unique. I also show that any Seifert fibre space admits a geometric structure. This yields a very natural and convenient 
division of closed Seifert fibre spaces into six types corresponding to six of the geometries. Finally, in $\$ 6$, I discuss briefly the progress made so far in proving Thurston's Geometrisation Conjecture.

None of the results of this article are really new, but the treatment of some of the topics is new, and some of the results have never appeared in print.

ACKNOWLeDGements. Most of the work on this article was done while I was visiting the University of Michigan. I was partially supported by NSF grant 81 02469. In addition, I would like to thank Terry Wall for his thorough reading of a preliminary draft of this article and for his many useful comments. Of course, the responsibility for any errors or omissions remains mine.

\section{§1. The 2-dimensional geometries}

There are three 2-dimensional geometries-namely the Euclidean plane $E^{2}$, the unit sphere $S^{2}$ in Euclidean 3-space and the hyperbolic plane $H^{2}$. The first two geometries are fairly well known but I shall spend a little time discussing them so as to bring out the similarities between them. The hyperbolic plane is much less familiar and often seems somewhat mysterious, so I will devote most of this section to an introduction to hyperbolic geometry. For each of the geometries, the main objects of interest will be discrete groups of isometries and the corresponding quotient surfaces.

Before getting down to details, I will say a little more about metrics. A metric on a space $X$ is simply a map $X \times X \rightarrow \mathbb{R}$ with certain properties, whereas a Riemannian metric on a manifold $M$ is a choice of inner product in each fibre of the tangent bundle of $M$. A Riemannian metric induces an ordinary metric on $M$. For the Riemannian metric assigns a length to any smooth path on $M$ which can be calculated as an integral, and one defines the distance apart of two points in $M$ to be the infimum of the lengths of all paths joining them. For our purposes, we can take this property of a Riemannian metric as the basic concept and can mostly ignore the refinements such as tangent bundles. Thus the standard Riemannian metric on the Euclidean plane $E^{2}$ can be given simply by the formula $d s^{2}=d x^{2}+d y^{2}$. This enables one to calculate the length of any smooth path $\gamma$ in $E^{2}$ as the integral $\int_{\gamma} d s$. It is a simple, but not quite trivial exercise to show that the metric which one obtains really is the standard Euclidean metric. This involves showing that given two points $P$ and $Q$ in $E^{2}$, then the straight line segment joining $P$ and $Q$ is shorter (strictly) than any other path from $P$ to $Q$ and has the required length.

The advantage of this approach becomes clear when one considers the metric on the unit sphere $S^{2}$ in $E^{3}$. Using the formula $d s^{2}=d x^{2}+d y^{2}+d z^{2}$ in $E^{3}$ one can assign a length to any path in $E^{3}$. In particular, given two points $P$ and $Q$ on $S^{2}$, one can find the length of each smooth path on $S^{2}$ joining $P$ and $Q$, and so define the distance from $P$ to $Q$ as the infimum of all these lengths. Clearly, this is what one wants for the standard metric on $S^{2}$. It is not difficult to show that there is a shortest path from $P$ to $Q$ and that such a path is an arc of a great circle. Thus great circles on $S^{2}$ play the same role as straight lines in $E^{2}$. I will use the word geodesic from now on to describe either of these. Note that a geodesic arc $l$ between points $P$ and $Q$ on $S^{2}$ need not be the shortest path from $P$ to $Q$ because $l$ may be more than half the 
length of the great circle to which it belongs. Note also that if $P$ and $Q$ are diametrically opposite, there are infinitely many geodesic arcs from $P$ to $Q$ all with the same length. The correct definition of a geodesic is a path which locally minimises length, i.e. if two points $A$ and $B$ on the path are close enough, then the geodesic segment $A B$ is the shortest path between $A$ and $B$.

Now we will return to the Euclidean plane and discuss some of the basic facts about discrete groups of isometries of $E^{2}$ and the corresponding quotient spaces. First, we consider the group of all isometries of $E^{2}$, sometimes called the Galilean group. I shall denote it by Isom $\left(E^{2}\right)$. It is not difficult to show that any isometry of $E^{2}$ is a translation, rotation, reflexion or glide-reflexion. (A glide-reflexion is the composite of a reflexion in a line $l$ with a translation along $l$.) In coordinate terms, any isometry $\alpha$ of $E^{2}$ can be expressed as $\alpha(\mathbf{x})=A \mathbf{x}+\mathbf{b}$ where $A$ is an orthogonal $2 \times 2$ matrix and $\mathbf{b}$ is a vector in $E^{2}$. We will not use this expression in coordinates, except to observe that the natural map Isom $\left(E^{2}\right) \rightarrow \mathrm{O}(2)$ sending $\alpha$ to $A$ is a surjective homomorphism with kernel equal to the subgroup consisting of all translations. This subgroup is isomorphic to $\mathbb{R}^{2}$ so that we have the exact sequence

$$
0 \longrightarrow \mathbb{R}^{2} \longrightarrow \operatorname{Isom}\left(E^{2}\right) \longrightarrow \mathrm{O}(2) \longrightarrow 1 \text {. }
$$

A convenient property of Isom $\left(E^{2}\right)$ is that it is generated by reflexions. To see this, consider the product of reflexions $\alpha$ and $\beta$ in lines $l$ and $m$ respectively. If $l$ and $m$ are parallel and distance $d$ apart, then $\alpha \beta$ is a translation through distance $2 d$ in a direction perpendicular to $l$ and $m$. If $l$ and $m$ intersect in a point $x$ at angle $\theta$, then $\alpha \beta$ is a rotation through angle $2 \theta$ about the point $x$. It follows that any translation and any rotation is a product of two reflexions. Hence the subgroup of Isom $\left(E^{2}\right)$ generated by all reflexions properly contains the orientation preserving subgroup and so must equal $\operatorname{Isom}\left(E^{2}\right)$.

We shall be interested in subgroups $G$ of $\operatorname{Isom}\left(E^{2}\right)$ such that $E^{2} / G$ is a reasonably nice surface. Thus we shall not consider, for example, the case when $G$ consists of all translations in a given direction as $E^{2} / G$ will then be 1-dimensional. We shall also exclude the case of an infinite cyclic group $G$ generated by a rotation through an irrational multiple of $\pi$. In this case, $E^{2} / G$ is an extremely nasty space. The usual condition which excludes such phenomena is discreteness.

We shall say that a group $G$ acting on a space $X$ acts properly discontinuously if for any compact subset $C$ of $X$,

$$
\{g \in G: g C \cap C \neq \varnothing\} \text { is finite } .
$$

Notice that if $G$ acts properly discontinuously on $X$, then the stabilizer of any point of $X$ must be finite. If all such stabilisers are trivial, we shall say that $G$ acts freely. It is not difficult to show that if $G$ acts freely and properly discontinuously on a manifold $X$, then the natural map $X \rightarrow X / G$ is a covering map with covering group $G$.

If $G$ acts properly discontinuously on a space $X$, then $G$ is a discrete subset of the space of all continuous functions $X \rightarrow X$ with the compact-open topology. The converse is false, in general, but is true if $X$ is a complete Riemannian manifold and $G$ is a group of isometries of $X$. It will be convenient to say that a group of isometries of such a manifold $X$ is discrete if it is a discrete subgroup of the isometry group of $X$. All the examples of group actions in this article will be of groups of isometries of 
complete Riemannian manifolds, so for brevity I shall say that a group $G$ is discrete or acts discretely instead of saying that $G$ acts properly discontinuously.

One can construct a group $G$ of isometries of $E^{2}$ which acts freely and discretely by taking $G$ to be generated by a translation or glide-reflexion or by taking $G$ to be generated by two translations or glide-reflexions in different directions. These are the only such examples. For any rotation or reflexion of $E^{2}$ has a fixed point. Thus it follows that if $G$ is a group of isometries of $E^{2}$ acting freely and effectively on $E^{2}$, then every element of $G$ is a translation or glide-reflexion. Thus the orientation preserving subgroup $G_{1}$ of $G$ consists only of translations and so is a discrete subgroup of $\mathbb{R}^{2}$, the group of all translations of $E^{2}$. Hence $G_{1}$ is isomorphic to $\mathbb{Z}$ or $\mathbb{Z} \oplus \mathbb{Z}$, and $G$ can be generated by at most two translations or glide reflexions. Notice that the quotient of $E^{2}$ by $G$, in these cases, is an open cylinder, an open Moebius band, a torus or a Klein bottle. As the projection $E^{2} \rightarrow E^{2} / G$ is a covering map, there is a natural identification of $G$ with the fundamental group of $E^{2} / G$. Finally, the quotient of $E^{2}$ by $G$ inherits a natural metric from the metric on $E^{2}$, as described in the introduction, such that the projection $E^{2} \rightarrow E^{2} / G$ is locally an isometry.

If $G$ is a discrete group of isometries of $E^{2}$ which does not act freely, then $G$ must contain an element of finite order, which is a rotation or reflexion. Before considering the general situation, we will consider the special cases where $G$ is cyclic or dihedral.

Let $G$ be the cyclic group generated by an isometry $\alpha$ which is a rotation through $2 \pi / n$ about a point 0 in $E^{2}$. It is clear that each orbit has exactly one point in the infinite wedge $W$ shown in Fig. 1.1, except that each point of $l_{1}$ lies in the same orbit as one point of $l_{2}$.
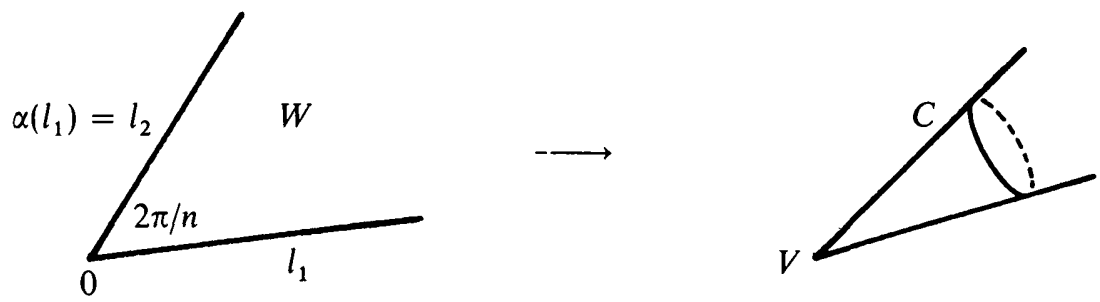

FIG. 1.1

It follows that the quotient space, $E^{2} / G$, which is obtained by identifying each orbit to a single point, is the same as the space obtained from $W$ by glueing $l_{1}$ to $l_{2}$, that is a cone $C$ with cone angle $2 \pi / n$ at a vertex which we call $V$. Topologically speaking, $C$ is simply another copy of $\mathbb{R}^{2}$, but it is important to keep thinking metrically. There is a natural metric on $C$ inherited from the metric on $E^{2}$. Any path $l$ in $C$ can be lifted to a path in $E^{2}$, although this lift is not necessarily determined by a lift of one endpoint of $l$ as it would be if the projection $E^{2} \rightarrow C$ was a covering map. This phenomenon occurs whenever $l$ goes through $V$. However, all lifts of $l$ will have the same length, so we can define the length of $l$ and hence define a metric on $C$. Clearly the projection $E^{2}-\{0\} \rightarrow C-V$ is a covering map and a local isometry. It is also clear that $C$ is isometric to a right circular cone embedded in $E^{3}$. The statement that the cone angle of $C$ is $2 \pi / n$ can be thought of as simply saying that a circle of radius $r$ on $C$ with centre $V$ has length $2 \pi r / n$. (A circle of radius $r$ centre at $V$ means the locus of all points in $C$ at distance $r$ from $V$. Clearly it is the projection of a circle of radius $r$ in $E^{2}$ with centre 0 .) 
If $G$ is a cyclic group of order two generated by reflexion in a line $l$, then $E^{2} / G$ again inherits a natural metric and is isometric to a half-plane whose boundary line is the image of $l$. See Fig. 1.2.

If $G$ is the dihedral group of order $2 n$ generated by a rotation of order $n$ about 0 and reflexion in a line through 0 , then $E^{2} / G$ again inherits a natural metric and is isometric to an infinite wedge with angle $\pi / n$. See Fig. 1.3.

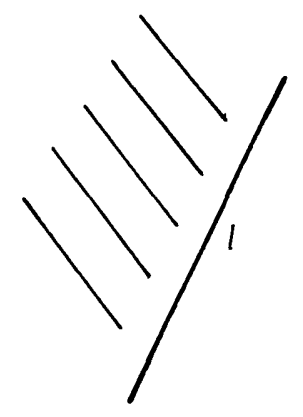

FIG. 1.2

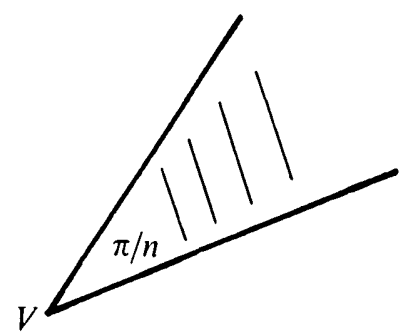

FIG. 1.3

In each of the above cases, we have described a natural metric on the quotient surface $E^{2} / G$. In the case when $G$ is cyclic and generated by a rotation, this metric has a singularity at the vertex $V$ of the cone $C$. In other words, the metric restricted to $C-\{V\}$ is Riemannian, but the metric on $C$ is not Riemannian. In the case when $G$ is generated by a reflexion, the quotient $E^{2} / G$ has a boundary line which we should think of as consisting of singular points. In the case when $G$ is dihedral, there are two semi-infinite boundary lines of singular points which meet in an even more singular point $V$ as in Fig. 1.3. These three types of singularity are called cone points, reflector lines and corner reflectors.

If we now consider a general discrete group of isometries of $E^{2}$, the situation is similar to the above. The quotient $E^{2} / G$ is a surface and inherits a natural metric from the metric on $E^{2}$. This metric will have singular points which can be of the above three types. A cone point on $E^{2} / G$ is a point with a neighbourhood isometric to a neighbourhood of $V$ in the cone $C$ of Fig. 1.1 for some angle $2 \pi / n$. If $E^{2} / G$ has non-empty boundary, the boundary of $E^{2} / G$ will consist of reflector lines with isolated corner reflector points.

As examples we consider the triangle groups. Let $p, q$ and $r$ denote three integers such that there is a triangle $\triangle$ in $E^{2}$ with angles $\pi / p, \pi / q$ and $\pi / r$. As this implies that $\frac{1}{p}+\frac{1}{q}+\frac{1}{r}=1$, it is not hard to show that the only possibilities for $p, q$ and $r$ are the triplets $(3,3,3),(2,3,6)$ and $(2,4,4)$. The full triangle group $\Delta^{*}(p, q, r)$ is defined to be the group of isometries of $E^{2}$ generated by the reflexions $L, M$ and $N$ in the three sides $Y Z, Z X$ and $X Y$ of $\triangle$. In each case, it is easy to see that the translates of $\triangle$ by $\triangle^{*}(p, q, r)$ tile $E^{2}$. This tiling is shown in Fig. 1.4 for the case $p=q=r=3$.

It is also easy to see that the stabiliser of $\Delta$ is trivial. Hence there is a bijective correspondence between the triangles in the tiling and the elements of $\triangle^{*}(p, q, r)$ defined by mapping $g$ in $\triangle^{*}(p, q, r)$ to the triangle $g \triangle$, as shown in Fig. 1.4. It is now clear that $\Delta^{*}(p, q, r)$ is discrete and that $E^{2} / \triangle^{*}(p, q, r)$ is isometric to the original triangle $\triangle$. 

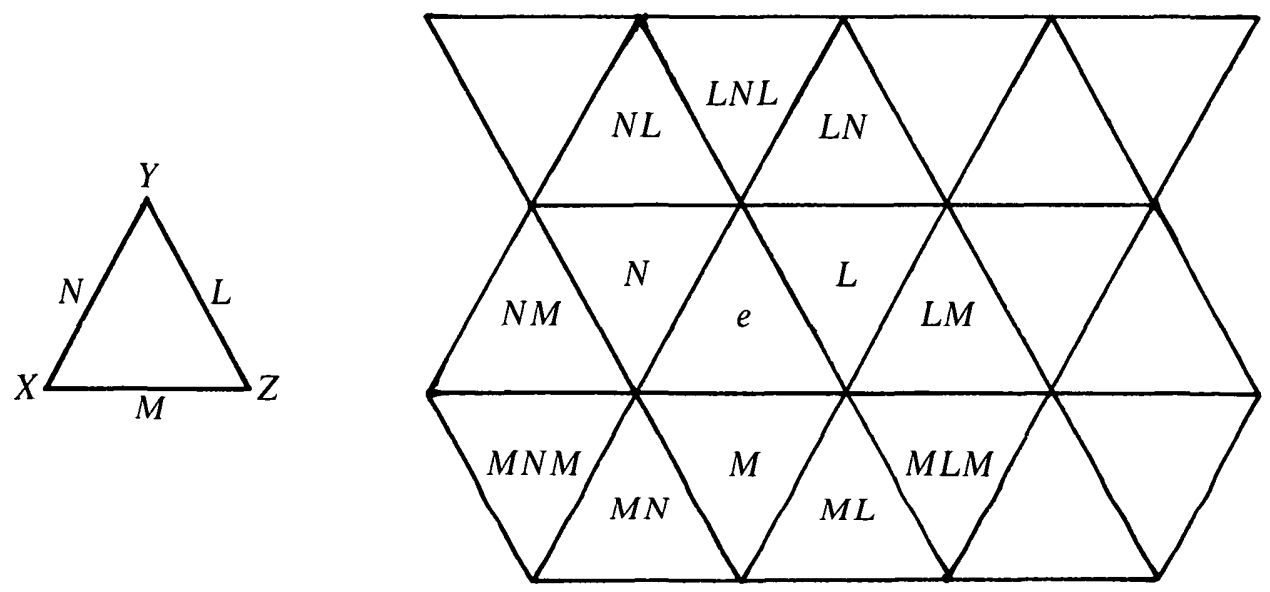

FIG. 1.4

One can make the same definitions starting with any triangle in $E^{2}$, but one is most unlikely to end up with a discrete group. If the triangle has angle $\alpha$ at a vertex $X$, then the group $\triangle^{*}$ generated by reflexions in the sides of the triangle contains a rotation about $X$ through angle $2 \alpha$. If $\Delta^{*}$ is to be discrete, this rotation must be of finite order, so that $\alpha$ must be a rational multiple of $\pi$. Further, the classification of discrete subgroups of Isom $\left(E^{2}\right)$ shows that either $\Delta^{*}$ is a finite group fixing a point of $E^{2}$ or every element of finite order in $\triangle^{*}$ has order $2,3,4$ or 6 . (See [50] or [75] and also see Table 4.4 in the section of this article on the geometry of $E^{3}$.) As $\Delta^{*}$ contains rotations about more than one point, namely the three vertices of the given triangle, it follows that $\Delta^{*}$ is infinite and so each angle of the triangle must be one of $\pi / 2, \pi / 3,2 \pi / 3, \pi / 4,3 \pi / 4, \pi / 6$ or $5 \pi / 6$. The only triangle in $E^{2}$ with such angles which does not have each angle of the form $\pi / n$, has angles $2 \pi / 3, \pi / 6, \pi / 6$. This triangle consists of two triangles with angles $\pi / 3, \pi / 2, \pi / 6$ and with a common edge. Hence $\Delta^{*}$ is a subgroup of $\triangle(2,3,6)$ in a natural way and is, in fact, equal to $\triangle(2,3,6)$. Hence there are only four triangles in $E^{2}$ (up to similarity) such that the group generated by reflexions in the edges is discrete.

For any group $G$ acting on a space $X$, we define a fundamental region for $G$ to be a closed subset $P$ of $X$ such that

and

$$
\bigcup_{g \in G} g P=X
$$

$$
\stackrel{\dot{P}}{P} \cap g \stackrel{\circ}{P}=\varnothing
$$

for all non-trivial elements $g$ in $G$, where $\stackrel{P}{P}$ denotes the interior $P$.

This definition is very general, but we will only consider polygonal fundamental regions and not worry about possible pathological examples. In the previous examples, $\triangle$ is a fundamental region for $\Delta^{*}(p, q, r)$.

Now $\Delta^{*}(p, q, r)$ has a natural subgroup of index two-the orientation preserving subgroup which is denoted $\triangle(p, q, r)$. The product $L M$ of two of the generating reflexions of $\triangle^{*}(p, q, r)$ is a rotation through $2 \pi / r$ about $Z$ and $M N$ and $N L$ are also rotations. Write $L M=z, M N=x, N L=y$ so that $x, y, z$ are 
rotations about $X, Y$, and $Z$ respectively. Clearly, $x, y$ and $z$ lie in $\Delta(p, q, r)$ and one can show that they generate it. Now $x y z=1$, from the definitions, so that $\Delta(p, q, r)$ can be generated by any two of $x, y$ and $z$. As $\Delta(p, q, r)$ contains rotations one should expect $E^{2} / \Delta(p, q, r)$ to have cone points. In fact, $E^{2} / \triangle(p, q, r)$ is topologically a 2 -sphere and has three cone points with cone angles $2 \pi / p, 2 \pi / q$ and $2 \pi / r$. The easiest way to see this is to observe that two adjacent triangles in the tiling of Fig. 1.4 must form a fundamental region for $\Delta(p, q, r)$. For example, if we let $P$ equal $\Delta \cup L \Delta$, then the translates of $P$ by $\Delta(p, q, r)$ cover $E^{2}$. For any $g$ in $\triangle^{*}(p, q, r)$, either $g$ lies in $\Delta(p, q, r)$ or in the coset $\Delta(p, q, r) \cdot L$, so any triangle $g \Delta$ lies in some translate of $P$ by an element of $\Delta(p, q, r)$. It follows that $E^{2} / \Delta(p, q, r)$ can be obtained from $P$ by appropriate identifications of edges. One can think of this quotient as a double of the original triangle $\Delta$, that is two copies of $\Delta$ have their boundaries identified.

This kind of construction for a fundamental region of a subgroup of finite index of a given group is very useful. If $G$ is a group of isometries of $E^{2}$ with fundamental region $X$ and if $G_{1}$ is a subgroup of $G$ of index $n$, then a fundamental region $X_{1}$ for $G_{1}$ can be formed from $n$ translates of $X$. If one makes translates of $X$ correspond with elements of $G$, as before, then $X_{1}$ is constructed by choosing coset representatives for $G_{1}$ in $G$ and taking the union of the corresponding translates of $X$. If $G$ is a discrete group of isometries of $E^{2}$ such that $E^{2} / G=F$ is compact, then the induced metric on $F$ gives $F$ an area. This area is clearly equal to the area of any fundamental region $X$ for $G$. Hence our discussion of fundamental regions shows that if $G_{1}$ is a subgroup of $G$ of index $n$, the area of $F_{1}=E^{2} / G_{1}$ is $n$ times the area of $F$. If the projection map $p: F_{1} \rightarrow F$ was a covering map this result would be obvious directly. In order to see directly that this result holds in all cases one need only observe that the projection fails to be a covering map only on a 1-dimensional set, i.e. if we let $W$ denote the union of all the singular points on $F$, then this has dimension at most one and the projection $F_{1}-p^{-1}(W) \rightarrow F-W$ is a covering map of degree $n$. The spaces $F-W$ and $F_{1}-p^{-1}(W)$ need not be connected, but this does not affect the argument.

There are very few discrete isometry groups of $E^{2}$ and these have been known for very many years. See [50] or [75]. Recall the exact sequence

$$
0 \longrightarrow \mathbb{R}^{2} \longrightarrow \operatorname{Isom}\left(E^{2}\right) \longrightarrow \mathrm{O}(2) \longrightarrow 1 \text {. }
$$

If $G$ is a subgroup of Isom $E^{2}$, let $T$ denote the subgroup of all translations in $G$. Then we must have the exact sequence below, where $H$ is a subgroup of $\mathrm{O}(2)$.

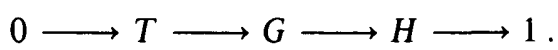

If $G$ is discrete, then $T$ must be trivial, $\mathbb{Z}$ or $\mathbb{Z} \oplus \mathbb{Z}$. If $T$ is trivial, then $G$ fixes a point of $E^{2}$ and must be finite cyclic or dihedral. If $T$ is cyclic, one obtains the seven strip pattern groups and if $T$ is $\mathbb{Z} \oplus \mathbb{Z}$, one obtains the seventeen wallpaper groups or crystallographic groups. It is interesting to list these groups by describing the various quotient spaces. The seventeen compact quotients are shown in Table 4.4 of the section of this article on the geometry of $E^{J}$ (page 446).

Next we consider the geometry of the 2-sphere $S^{2}$ with the metric induced from its embedding in $E^{3}$. As discussed earlier, the great circles on $S^{2}$ are the geodesics on 
$S^{2}$ and so play the same role for the geometry of $S^{2}$ as do the straight lines for the geometry of $E^{2}$. However, there are some very important differences which need discussing before we consider isometries of $S^{2}$. The two most obvious differences are that a geodesic on $S^{2}$ is a circle and not a line and that any two geodesics on $S^{2}$ must meet-there is no such thing as parallel geodesics on $S^{2}$. Another difference is that any two points on $E^{2}$ lie on a unique geodesic whereas diametrically opposite points on $S^{2}$ lie on infinitely many geodesics. (Otherwise two points on $S^{2}$ do lie on a unique geodesic.)

Perhaps the most crucial difference is the effect that the curvature of $S^{2}$ has on the properties of areas of polygons. If we consider triangles (with geodesic sides) drawn on $E^{2}$ and $S^{2}$, one knows that specifying the three angles of a triangle in $E^{2}$ does not determine its area. One can have very small or very large triangles with given angles. However, on $S^{2}$, the angles of a triangle determine its area completely. If a triangle on $S^{2}$ has angles $\alpha, \beta$ and $\gamma$, then the area of the triangle is $\alpha+\beta+\gamma-\pi$. Note that on a sphere of radius $R$, this would be replaced by $R^{2}(\alpha+\beta+\gamma-\pi)$. This can be proved by very elementary arguments starting from the obvious fact that the area of one of the regions between two geodesics on a sphere $\Sigma$ meeting at an angle $\alpha$ is $(\alpha / 2 \pi) \times($ area of $\Sigma)$. A more fancy argument is to use the Gauss-Bonnet Theorem as $S^{2}$ has curvature 1 . This formula shows at once that any triangle drawn on $S^{2}$ has angle sum greater than $\pi$ and that this sum can vary greatly. In fact it can take any value between $\pi$ and $5 \pi$, though, of course, large angle sums require one to consider triangles with some or all angles greater than $\pi$. By subdividing into triangles, one can see that the angle sum of an $n$-gon on $S^{2}$ must always exceed $(n-2) \pi$, which is the angle sum of an $n$-gon in $E^{2}$. One question here is whether, given a collection of $n$ angles whose sum exceeds $(n-2) \pi$, one can find a polygon in $S^{2}$ with these angles. Certainly, if all angles are less than $\pi$, this can be done.

The full isometry group of $S^{2}$ is $\mathrm{O}(3)$, the group of $3 \times 3$ orthogonal matrices, which is the group of isometries of $E^{3}$ fixing the origin 0 . An element of $\mathrm{SO}(3)$ is a rotation of $E^{3}$ about some line through 0 . The restriction to $S^{2}$ is also called a rotation and will fix exactly two points on $S^{2}$. Thus every orientation preserving isometry of $S^{2}$ is a rotation. The orientation reversing elements of $\mathrm{O}(3)$ are of two types depending on whether or not they have an eigenvalue equal to 1 . Let $\alpha$ be an orientation reversing element of $\mathrm{O}(3)$. Such an element must have an eigenvalue equal to -1 . Hence there is a line $l$ in $E^{3}$ through 0 which is left invariant by $\alpha$ but has its sense reversed. The orthogonal plane $\Pi$ through 0 is invariant under $\alpha$ and the restriction of $\alpha$ to $\Pi$ must be a rotation. If this rotation is trivial, then $\alpha$ is reflexion in $\Pi$. Otherwise $\alpha$ fixes only the origin. Thus there are two kinds of orientation reversing isometry of $S^{2}$-reflexions, which fix a great circle in $S^{2}$, and isometries without fixed points. Recall that the great circles on $S^{2}$ are the geodesics, so that a reflexion of $S^{2}$ fixes a unique geodesic in the same way that a reflexion of $E^{2}$ fixes a unique straight line. We will therefore talk of reflexion in a geodesic $l$ on $S^{2}$ in the same way as we refer to reflexions in a line in $E^{2}$. As for $E^{2}$, the isometry group of $S^{2}$ is generated by reflexions. To see this, let $\alpha$ and $\beta$ be reflexions of $S^{2}$ in geodesics $l$ and $m$ respectively. Unlike the situation for $E^{2}$, two geodesics on $S^{2}$ cannot be disjoint. They must be equal or intersect in two points $x$ and $y$ at some angle $\theta$. Hence if $\alpha$ and $\beta$ are distinct, the product $\alpha \beta$ is a rotation of $S^{2}$ through angle $2 \theta$ and fixing $x$ and $y$. It follows that any rotation of $S^{2}$ is a product of two reflexions, so that the subgroup of $\mathrm{O}(3)$ generated by reflexions properly contains $\mathrm{SO}(3)$ and so must equal $\mathrm{O}(3)$. 
Now we briefly consider discrete isometry groups of $S^{2}$. The definition of discreteness shows at once that if $G$ acts on $S^{2}$ then $G$ is discrete if and only if $G$ is finite. There is a unique non-trivial subgroup of $\mathrm{O}(3)$ which acts freely on $S^{2}$, namely the group of order two generated by the antipodal map of $S^{2}$. For if $G$ acts on $S^{2}$, every orientation preserving element is a rotation and so has fixed points. Hence if $G$ acts freely on $S^{2}$, it must have order two, and one can check easily that the antipodal map is the only fixed point free isometry of $S^{2}$ which has order two. The quotient of $S^{2}$ by this group is the real projective plane $P^{2}$, and, as usual, $P^{2}$ inherits a natural metric from $S^{2}$ such that the projection $S^{2} \rightarrow P^{2}$ is a local isometry.

If $G$ is a discrete (hence finite) group acting non-freely on $S^{2}$, the general picture is as for discrete groups acting on $E^{2}$. The quotient $S^{2} / G$ inherits a natural metric which has singularities at three types of point-cone points, reflexion points and corner reflector points. In a neighbourhood of each of these points, one has a standard picture obtained by considering the cases where $G$ is cyclic or dihedral as was the case for groups acting on $E^{2}$. Of course, if $G$ is a cyclic group of rotations acting on $S^{2}$ fixing points $x$ and $y$, then a neighbourhood of $V$, the projection of $x$, in $S^{2} / G$ is not isometric to a right circular cone in $E^{3}$. The picture, as shown in Fig. 1.5, is of a cone-like object but with the same curvature as $S^{2}$ at all points apart from the cone point. One can recognise that such a cone point has cone angle $2 \pi / n$ because the length of a circle of radius $r$ in $S^{2} / G$ centred on $V$ is $1 / n$ times the length of a circle of radius $r$ in $S^{2}$.
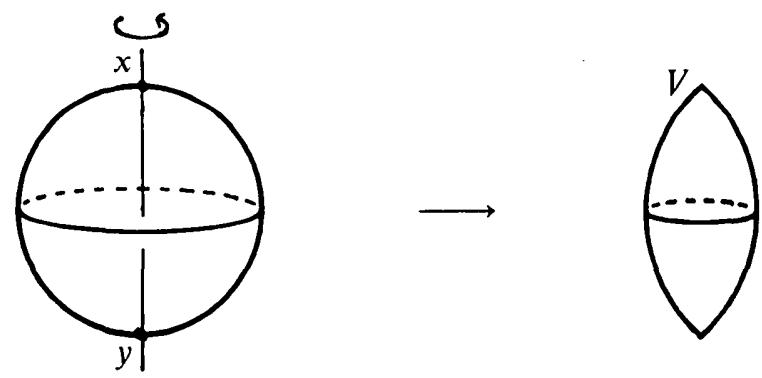

F1G. 1.5

The tinite subgroups of SO (3) are completely classified. See [75]. Any such group must be cyclic, dihedral or the orientation preserving symmetry group of one of the five regular solids. Note that the symmetry groups of the icosahedron and dodecahedron are naturally isomorphic as are the symmetry groups of the cube and octahedron. The quotient of $S^{2}$ by a cyclic group is illustrated in Fig. 1.5, and is a 2-sphere with two cone points. The quotient of $S^{2}$ by a dihedral group acting orientation preservingly is a 2-sphere with three cone points and so is the quotient of $S^{2}$ by the orientation preserving symmetry groups of the regular solids. In fact, these groups are all triangle groups and can be described in the same way as Euclidean triangle groups.

If $p, q$ and $r$ are integers such that $\frac{1}{p}+\frac{1}{q}+\frac{1}{r}>1$, there is a triangle (unique up to congruence) on $S^{2}$ with angles $\pi / p, \pi / q$ and $\pi / r$. One can show easily that the triple $(p, q, r)$ must be of the form $(2,2, n),(2,3,3),(2,3,4)$ or $(2,3,5)$. One defines $\Delta^{*}(p, q, r)$ to be the group of isometries of $S^{2}$ generated by reflexions in the sides of the triangle and $\Delta(p, q, r)$ to be the orientation preserving subgroup. Then $\Delta(2,2, n)$ 
is dihedral of order $2 n, \Delta^{*}(2,3,3)$ is the full symmetry group of the tetrahedron, $\Delta^{*}(2,3,4)$ is the full symmetry group of the cube (or octahedron) and $\Delta^{*}(2,3,5)$ is the full symmetry group of the dodecahedron (or icosahedron). I shall explain the isomorphism for $\Delta^{*}(2,3,3)$. The others are very similar. Start with a regular tetrahedron in $E^{3}$ with vertices on $S^{2}$ and project from the origin to obtain a tiling of $S^{2}$ by four congruent, equilateral triangles, i.e. a spherical tetrahedron. Each angle in these triangles must be $2 \pi / 3$, as three such angles fit round each vertex. Now subdivide this triangulation of $S^{2}$ by adding a vertex at the midpoint of each edge and at the centroid of each triangle. This gives a tiling of $S^{2}$ by 24 congruent triangles with angles $\pi / 2, \pi / 3$ and $\pi / 3$. By picking one of these triangles we have a natural identification of $\triangle^{*}(2,3,3)$ with the symmetry group of the tetrahedron.

Now we can consider the hyperbolic plane $H^{2}$. One of the problems here is to be sure that $H^{2}$ really exists. See Milnor's article [39], for the history of hyperbolic geometry and for further references. One cannot visualise the hyperbolic plane in the same way that one can visualise $E^{2}$ and $S^{2}$. There are various approaches to the construction of $H^{2}$. I shall take a differential geometric approach based on the idea that the hyperbolic plane is a curved surface just as $S^{2}$ is. Note that $S^{2}$ has the same curvature at all points. In fact, the isometry group $\mathrm{O}(3)$ of $S^{2}$ acts transitively on $S^{2}$. The idea is that $H^{2}$ also has the same curvature at all points but that it "curves in the opposite sense". Unfortunately $H^{2}$ cannot be isometrically embedded in $E^{3}$ but locally it is isometric to a surface with a saddle point at every point. A very nice example is the pseudo-sphere, which is the surface of revolution of a tractrix, shown in Fig. 1.6. This surface clearly has a saddle point at every point and it is also true that the curvature is the same at every point. However, this surface is neither simply connected nor complete.

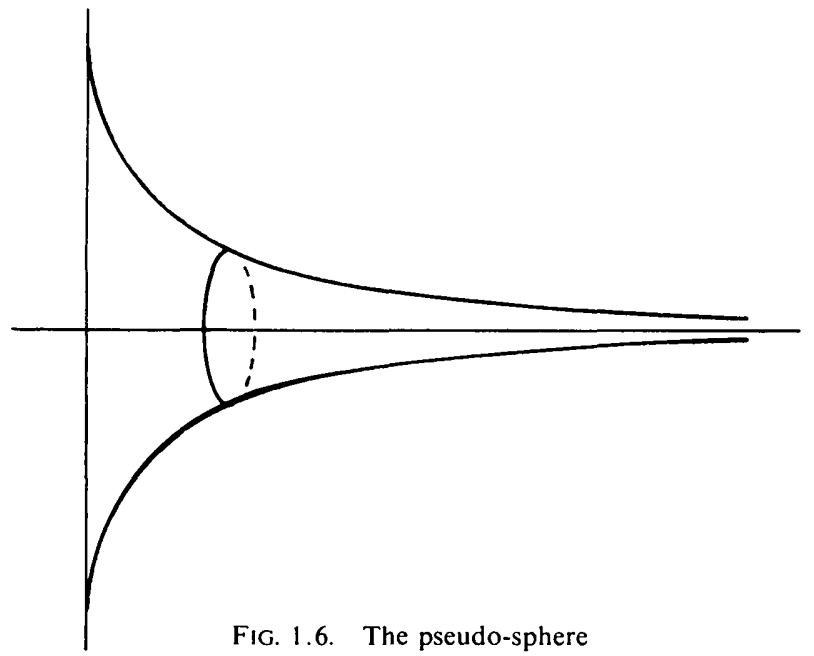

To give a precise description of $H^{2}$, we turn to the idea of a map (as in cartography, not as in function). If one looks in an atlas at a map of a large portion of the Earth's surface, one finds certain distortions caused by the fact that the atlas is flat and the Earth is not flat. The scale of the map will alter as one moves around the map. A geodesic on the Earth's surface may appear to be curved on the map and conversely a straight line on the map may not represent a geodesic on the Earth's surface. 
There is a homeomorphism between the map and part of the Earth's surface, so the metric on the Earth's surface induces a metric on the map. If the Earth were flat and if we identify our map with the plane, then this metric would be given by the formula $d s^{2}=N^{2}\left(d x^{2}+d y^{2}\right)$ where the scale of the map is $N$ to 1 . As the Earth is not flat, the corresponding metric on the plane will be given by a formula like $d s^{2}=a d x^{2}+2 b d x d y+c d y^{2}$ where $a, b$ and $c$ are functions of $x$ and $y$. The plane with this peculiar metric will be isometric to (part of) the Earth's surface, by construction. I plan to reverse this process by starting with the plane with a certain metric. It seems an attractive idea to think of this as a map of the hyperbolic plane in the above sense, but really one should regard this as a definition of the hyperbolic plane.

To define $H^{2}$, we start with the upper half plane $\mathbb{R}_{+}^{2}=\left\{(x, y) \in \mathbb{R}^{2}: y>0\right\}$ and the formula $d s^{2}=\frac{1}{y^{2}}\left(d x^{2}+d y^{2}\right)$. As discussed before, this assigns a length to each smooth path in $\mathbb{R}_{+}^{2}$ and hence defines a metric on $\mathbb{R}_{+}^{2}$. We need to know which curves in $H^{2}$ are geodesics in order to begin to understand the geometry of $H^{2}$. There are fundamental theorems of differential geometry which assure us that there must be geodesics in $H^{2}$, but one can avoid any use of these results by being a little careful. The first step is to show that vertical straight lines in $\mathbb{R}_{+}^{2}$, that is lines $x=$ constant, are geodesics of $H^{2}$. Let $P_{0}$ and $P_{1}$ be two points of $\mathbb{R}_{+}^{2}$ with the same $x$-coordinate. Let their $y$-coordinates be $y_{0}$ and $y_{1}$ respectively. Then the length of the vertical straight line segment $\gamma$ from $P_{0}$ to $P_{1}$ is

$$
\int_{;} d s=\int_{y_{0}}^{y_{1}} \frac{1}{y} d y=\log \left|\frac{y_{1}}{y_{0}}\right|
$$

because $d s=\frac{1}{y} d y$ on $\gamma$. If $l$ is a different path from $P_{0}$ to $P_{1}$, then the length of $l$ must be strictly greater than this. To see this, parametrise $l$ by $t$ so that $l$ has length $\int_{t_{0}}^{t_{1}} \frac{1}{y}\left(\left(\frac{d x}{d t}\right)^{2}+\left(\frac{d y}{d t}\right)^{2}\right)^{\frac{1}{2}} d t$.' As $\frac{d x}{d t}$ is non-zero at some point, one has

$$
\text { length of } l>\int_{t_{0}}^{t_{1}} \frac{1}{y}\left(\left(\frac{d y}{d t}\right)^{2}\right)^{\frac{1}{2}} d t \geqslant \text { length of } \gamma .
$$

It follows that all vertical lines in $\mathbb{R}_{+}^{2}$ are geodesics of $H^{2}$. It also follows, from the above, that if $P_{0}$ and $P_{1}$ are any two points on such a line, then the vertical line segment from $P_{0}$ to $P_{1}$ is the unique shortest path from $P_{0}$ to $P_{1}$. Finally, it follows that these vertical lines are doubly infinite in length despite the boundedness (in Euclidean terms) of $\mathbb{R}_{+}^{2}$ at the $x$-axis.

The second step is to find some isometries of $H^{2}$. As the translate of a geodesic by an isometry must be another geodesic, this will give us more geodesics. One obvious kind of isometry of $H^{2}$ is to reflect $\mathbb{R}_{+}^{2}$ in a vertical straight line. Clearly such a map is an isometry of $H^{2}$. The product of two such is a horizontal translation of $\mathbb{R}_{+}^{2}$ and this is also an isometry of $H^{2}$. A less obvious isometry is inversion of $\mathbb{R}_{+}^{2}$ in a semi- 
circle of radius $r$ centred at a point $a$ on the $x$-axis. Let $f: \mathbb{R}_{+}^{2} \rightarrow \mathbb{R}_{+}^{2}$ denote this map and write $(u, v)=f(x, y)$. One shows that $f$ is an isometry by checking that $\frac{1}{y^{2}}\left(d x^{2}+d y^{2}\right)=\frac{1}{v^{2}}\left(d u^{2}+d v^{2}\right)$. This calculation is simplified by using complex number notation. One writes $z=x+i y, \quad w=f(z)$ and one needs to check that $\frac{d z \overline{d z}}{(\bar{z}-z)^{2}}=\frac{d w \overline{d w}}{(\bar{w}-w)^{2}}$ where

$$
w-a=\frac{r^{2}}{\bar{z}-a} .
$$

This will be left to the reader.

It is well known that inversion of the plane in a circle preserves angles and sends straight lines and circles to straight lines or circles. A simple proof of the second fact can be given by using polar coordinates with origin at the centre of inversion. Any circle or straight line in the plane has equation $a r^{2}+b r \cos \theta+c r \sin \theta+d=0$, where the case $a=0$ corresponds to straight lines. Now inversion sends $(r, \theta)$ to $\left(e^{2} / r, \theta\right)$ where $e$ is the radius of inversion and it is clear that the new equation has the same form. I will discuss angles later. As angles are reversed in sense, an inversion is said to be anti-conformal. It follows that the image of a vertical straight line in $\mathbb{R}_{+}^{2}$ under inversion in a circle centred on the $x$-axis is a semi-circle meeting the $x$-axis orthogonally. Any such semi-circle is the image of a vertical straight line under such an inversion. For example, the semi-circle with ends at $a$ and $b$ on the $x$-axis can be obtained from the line $x=a$ by inverting in the semi-circle with centre at $b$ and radius $|a-b|$. It follows that every semi-circle in $\mathbb{R}_{+}^{2}$ centred at a point on the $x$-axis is a geodesic of $H^{2}$.

The isometries used above all leave fixed (pointwise) a geodesic of $H^{2}$ and interchange the sides of the geodesic, so it is natural to call them reflexions of $H^{2}$. We need to show that the full isometry group of $H^{2}$ is generated by these reflexions and that there are no more geodesics in $H^{2}$ than described above.

If we consider any two points of $\mathrm{H}^{2}$, there is a unique geodesic of $\mathrm{H}^{2}$, of the type discussed above, which passes through both of them. Now suppose that there is a geodesic $l$ on $H^{2}$ which is not of the type already described. We can find two points $P$ and $Q$ on $l$ such that the segment of $l$ between $P$ and $Q$ is shorter (perhaps not strictly) than any other path from $P$ to $Q$. There is also a geodesic $m$ through $P$ and $Q$ where $m$ is a vertical straight line or a semi-circle, and by applying a reflexion to $H^{2}$, if necessary, we can suppose that $m$ is a vertical straight line. But we showed earlier that the segment of $m$ between $P$ and $Q$ is strictly shorter than any other path from $P$ to $Q$. This contradicts the fact that the segment of $l$ between $P$ and $Q$ is shorter than any other path from $P$ to $Q$. It follows that every geodesic of $H^{2}$ is a vertical straight line or a semi-circle centred on the $x$-axis. As for $E^{2}$, any pair of points in $H^{2}$ lies on a unique geodesic and two distinct geodesics meet in at most one point. The big difference from $E^{2}$ is that given a geodesic $l$ in $H^{2}$ and a point $P$ not on $l$, there are infinitely many geodesics through $P$ which do not meet $l$. See Fig. 1.7. Hence the term non-Euclidean geometry.

Before completing our discussion of isometries of $H^{2}$, we need to spend a little time talking about angles and triangles. Let $A$ and $B$ be curves in $E^{2}$ which meet at a point $P$. Let $\mathbf{a}$ and $\mathbf{b}$ be non-zero vectors tangent at $P$ to $A$ and $B$ respectively. Then the angle $\theta$ between $A$ and $B$ at $P$ is defined to be the angle between $\mathbf{a}$ and $\mathbf{b}$, and this 
has cosine equal to $\frac{\mathbf{a} \cdot \mathbf{b}}{a b}$, where one uses the usual scalar product for vectors in $E^{2}$ and where $a$ and $b$ are the lengths of $\mathbf{a}$ and $\mathbf{b}$ respectively. Thus

$$
\cos \theta=\frac{\mathbf{a} \cdot \mathbf{b}}{(\mathbf{a} \cdot \mathbf{a})^{\frac{1}{2}}(\mathbf{b} \cdot \mathbf{b})^{\frac{1}{2}}}
$$

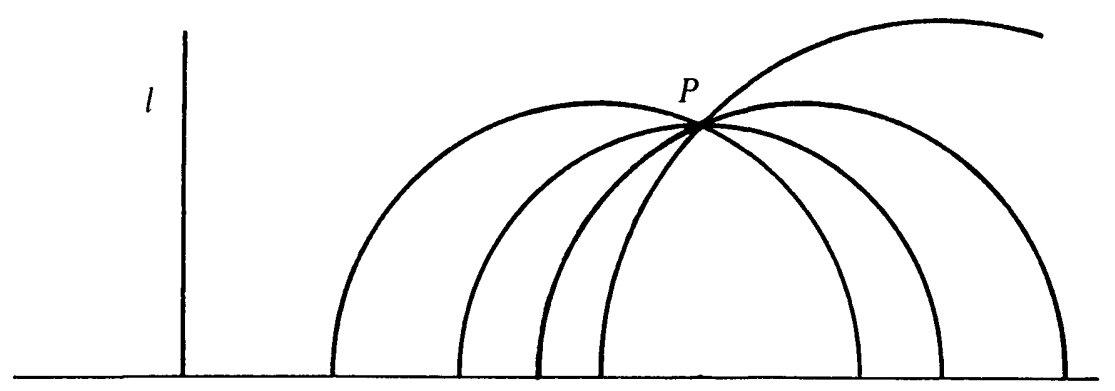

FIG. 1.7. Infinitely many geodesics in $H^{2}$ pass through $P$ and do not meet $I$

One defines the angle between two curves which meet at a point $P$ in a Riemannian manifold $M$ by the same formula, where the inner product to be used is the inner product which the Riemannian metric associates to the tangent space $T_{P}$ of $M$ at $P$. Now if $P$ is the point $\left(x_{0}, y_{0}\right)$ in $\mathbb{R}_{+}^{2}$, then the inner product on $T_{P}$ from the hyperbolic metric is simply $\frac{1}{y_{0}^{2}}$ times the standard inner product for $E^{2}$. It follows at once that the angle between two curves in $\mathbb{R}_{+}^{2}$ is the same whether it is measured in $H^{2}$ or $E^{2}$. Thus our map of the hyperbolic plane does not distort angles. These remarks also explain why inversion of the Euclidean plane preserves angles but reverses their sign. In terms of tangent spaces, the point is that if $f(z)=\frac{R^{2}}{\bar{z}}$ is an inversion of the plane in a circle of radius $R$ centred at the origin and if $\mathbf{a}$ is a point on the $x$-axis, then the differential of $f$ at $\mathbf{a}$, which is a linear map $\mathbb{R}^{2} \rightarrow \mathbb{R}^{2}$, is simply given by a matrix of the form $\left(\begin{array}{rr}\lambda & 0 \\ 0 & -\lambda\end{array}\right)$.

Now we consider triangles in $H^{2}$ with geodesic edges. The situation here is similar to that for triangles in $S^{2}$, that is there is a formula for the area of a triangle with angles $\alpha, \beta, \gamma$. The area is $\pi-(\alpha+\beta+\gamma)$. The proof of this is a simple exercise in integration. If $\triangle$ is a triangle in $H^{2}$, its area is $\iint_{\Delta} \frac{1}{y^{2}} d x d y$, because all lengths in $H^{2}$ are (locally) simply $\frac{1}{y}$ times lengths in $E^{2}$. It helps to use isometries of $H^{2}$ to arrange that two of the vertices of $\Delta$ are vertically above each other. It follows that any triangle in $H^{2}$ has area less than $\pi$, which is remarkable when compared with the Euclidean situation. It also follows that any triangle in $H^{2}$ has angle sum less than $\pi$. Conversely, given three positive numbers $\alpha, \beta$ and $\gamma$ such that $\alpha+\beta+\gamma<\pi$, there is a triangle in $H^{2}$ with angles $\alpha, \beta$ and $\gamma$. In fact, this triangle is unique up to congruence i.e. given triangles $A B C$ and $A^{\prime} B^{\prime} C^{\prime}$ in $H^{2}$ with angles $\alpha, \beta$ and $\gamma$ there is an isometry of $H^{2}$ throwing one triangle onto the other. One can find such an 
isometry as follows. There is a reflexion of $H^{2}$ sending $A^{\prime}$ to $A$. Next, there is a reflexion of $H^{2}$ in a line through $A$ which makes the edges $A B$ and $A^{\prime} B^{\prime}$ coincide. If $A C$ and $A^{\prime} C^{\prime}$ do not also coincide, we use a further reflexion in the line $A B$. We now have the situation shown diagrammatically in Fig. 1.8(i) or (ii), unless $B=B^{\prime}$ and $C=C^{\prime}$, which is what we want to prove.

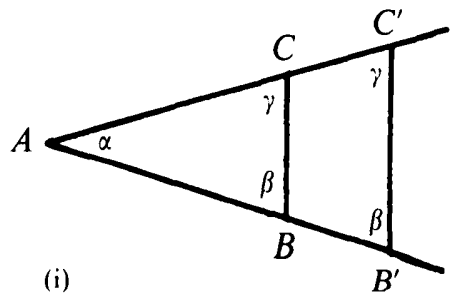

FIG. 1.8

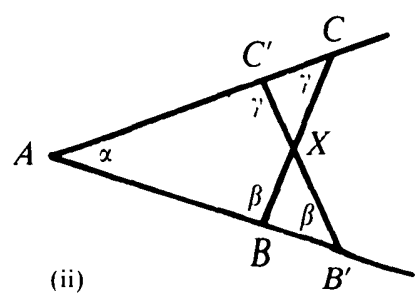

(ii)

In Fig. 1.8(i), the quadrilateral $B B^{\prime} C^{\prime} C$ has angle sum $2 \pi$. This contradicts our earlier observation that on $H^{2}$ an $n$-gon has angle sum less than $(n-2) \pi$. In Fig. 1.8(ii), the triangle $X C C^{\prime}$ has angle sum greater than $\pi$, which is also impossible. These contradictions show that $B$ and $B^{\prime}$ must coincide and so must $C$ and $C^{\prime}$ coincide.

Now we are in a position to show that any isometry of $H^{2}$ is a product of the reflexions described previously. Note that given two points $x, y$ of $H^{2}$, there is a reflexion which interchanges them. Note also that through a point of $H^{2}$, there is precisely one geodesic in each direction. Let $\alpha$ be an isometry of $H^{2}$ and pick a point $P$ in $H^{2}$. If $\alpha$ does not fix $P$, we compose $\alpha$ with a reflexion $\sigma$ sending $\alpha(P)$ to $P$ so that $\beta=\sigma \circ$ a fixes $P$. Now fix a geodesic $l$ through $P$. If $\beta$ leaves $l$ invariant and preserves the ends of $l$ then $\beta$ must fix $l$ pointwise as a point on $l$ is then determined by its distance from $P$. Otherwise, we compose $\beta$ with a reflexion $\tau$ in a geodesic through $P$ to obtain $\gamma=\tau \circ \beta$ such that $\gamma$ fixes $l$ pointwise. If $\gamma$ interchanges the sides of $l$ we compose $\gamma$ with reflexion in $l$. The result we need to complete the argument is that if $\gamma$ is an isometry of $H^{2}$ fixing $l$ pointwise and not interchanging the sides of $l$ then $\gamma$ is the identity. To see this let $m$ be any geodesic through $P$. Then $\gamma$ must preserve $m$ as angles are preserved and hence $\gamma$ fixes $m$ pointwise. As every point of $H^{2}$ lies on a geodesic through $P$, the result follows. Note that the above argument shows that any isometry of $H^{2}$ is a product of at most three reflexions.

The isometry group of $H^{2}$ acts transitively confirming the claim that $H^{2}$ has constant curvature. The stabiliser of a point $P$ is naturally isomorphic to $O(2)$, the stabiliser of a point in $E^{2}$, and is generated by all reflexions in lines through $P$. As every isometry of $H^{2}$ is a product of at most three reflexions, the orientation preserving subgroup consists of products of pairs of reflexions. It is a simple calculation to show that the product of any two reflexions of $H^{2}$ has the form $z \rightarrow \frac{a z+b}{c z+d}$ where $a, b, c$ and $d$ are real and $a d-b c>0$. Conversely one can show directly that any such transformation is a product of two reflexions or one can verify by another calculation that any such transformation is an isometry of $H^{2}$. (Note that a transformation of $\mathbb{C} \cup\{\infty\}$ of the form $z \rightarrow \frac{a z+b}{c z+d}$ where $a, b, c, d \in \mathbb{C}$ and $a d-b c \neq 0$, is called a Moebius transformation. If $a, b, c$ and $d$ are real and $a d-b c<0$, then the corresponding Moebius transformation interchanges $\mathbb{R}_{+}^{2}$ and $\mathbb{R}_{-}^{2}$.) It follows that the orientation preserving subgroup of $\mathrm{H}^{2}$ can be identified with 
the group $\operatorname{PSL}(2, \mathbb{R})$, which is the quotient of $\operatorname{SL}(2, \mathbb{R})$ by its central subgroup of order two. Now this group is a subgroup of $\operatorname{PSL}(2, \mathbb{C})$, the group of all Moebius transformations of $\mathbb{C}$, and this enables us to find another rather convenient model or map of the hyperbolic plane. The Moebius transformation $z \rightarrow \frac{z-i}{z+i}$ maps $\mathbb{R}_{+}^{2}$ conformally onto the interior $U$ of the unit disc in $\mathbb{R}^{2}$. The metric on this disc induced from the hyperbolic metric on $\mathbb{R}_{+}^{2}$ is $d s^{2}=\frac{4}{\left(1-\left(x^{2}+y^{2}\right)\right)^{2}}\left(d x^{2}+d y^{2}\right)$, and $U$ with this metric is another model of $H^{2}$. The geodesics of $H^{2}$ are arcs of circles which meet $\partial U$ orthogonally (such a "circle" through the origin will be a straight line) and the orientation preserving isometries of $\mathrm{H}^{2}$ are those Moebius transformations which leave $U$ invariant.

As I remarked earlier, the geodesics of $H^{2}$ are infinitely long despite the fact that $U$ is bounded in Euclidean terms. This enables us to think of the unit circle in $\mathbb{R}^{2}$, which bounds $U$, as a circle at infinity in $H^{2}$. Each geodesic of $H^{2}$ clearly has a point at infinity at each end. We can now talk of geodesics meeting at infinity. If two geodesics meet at infinity they are sometimes called "parallel" by analogy with the Euclidean situation. However, be warned that this in only an analogy. Two distinct parallel geodesics in $H^{2}$ diverge exponentially as one moves away from the common point at infinity. Also, if one chooses three points at infinity, they determine a "triangle" in $H^{2}$ with vertices at these three points. The edges of the "triangle" are geodesics of $H^{2}$ and the vertices are at infinity. Such a "triangle" is called an ideal triangle and in many ways can be treated on equal terms with ordinary triangles. One can also have a triangle with some but not all of its vertices at infinity. If wr take the angle at the infinite vertices to be zero, then the area formula remains true. Thus an ideal triangle has area $\pi$ and all other triangles have area strictly less than $\pi$.

Two geodesics which do not meet at all, even at infinity, are called ultra-parallel. A useful fact here is that two ultra-parallel geodesics have a unique common perpendicular. Notice that two parallel geodesics cannot have a common perpendicular as this would give rise to a triangle with angles $\pi / 2, \pi / 2$ and zero, which is impossible by the area formula. The existence and uniqueness of a common perpendicular for two ultra-parallel geodesics $l$ and $m$ is clear from Fig. 1.9.

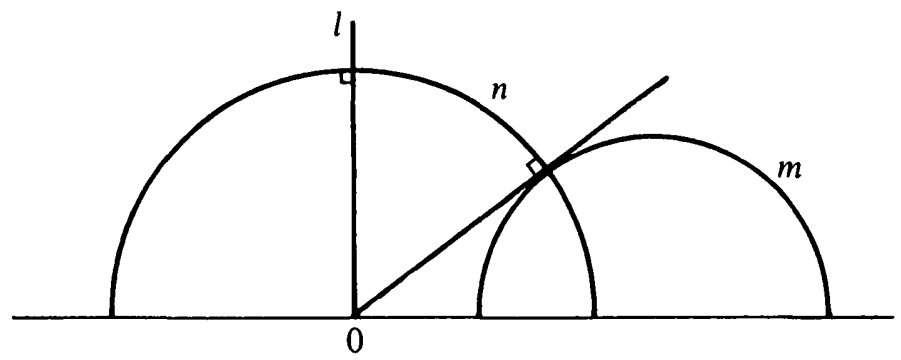

FIG. 1.9

In Euclidean terms, $n$ is a semi-circle with centre at 0 and its radius is such that it meets $m$ at the same point as the tangent to $m$ from 0 .

We can now give a description of the different types of isometry of $H^{2}$. If $\alpha$ is an orientation preserving isometry of $H^{2}$, then $\alpha$ is the product of two reflexions in geodesics $l$ and $m$, and there are three different cases according to the relative positions of $l$ and $m$ in $H^{2}$. If $l$ and $m$ intersect in a point $P$ of $H^{2}$, then $\alpha$ fixes $P$ and is 
called a rotation about $P$. The angle of rotation is twice the angle between $l$ and $m$. If $P$ is the origin in the disc model for $H^{2}$, then $\alpha$ is a rotation, in Euclidean terms. It clearly fixes no points at infinity. If $l$ and $m$ meet at infinity, or are parallel, then the simplest picture is obtained by taking their meeting point to be $\infty$ in the upper half plane model of $H^{2}$. Now $l$ and $m$ are vertical straight lines and $\alpha$ is a horizontal translation of $\mathbb{R}_{+}^{2}$. Such an isometry is called parabolic. Note that $\alpha$ fixes exactly one point at infinity. Finally, if $l$ and $m$ are ultra-parallel, the situation becomes like the Euclidean one in that $\alpha$ leaves invariant the unique common perpendicular $n$ of $l$ and $m$ and $\alpha$ translates $n$ a distance $2 d$ where $d$ is the perpendicular distance from $l$ to $m$. This isometry fixes exactly two points at infinity. In particular, $n$ is the only geodesic left invariant by $\alpha$. Such an isometry is called hyperbolic or a translation along $n$.

If $\alpha$ is orientation reversing, then $\alpha$ induces an orientation reversing homeomorphism of $S^{1}$, the circle at infinity of $H^{2}$. Hence $\alpha$ fixes at least two points of $S^{1}$ and so leaves a geodesic $l$ invariant. If we compose $\alpha$ with reflexion $\sigma$ in $l$ we obtain an orientation preserving isometry $\beta=\sigma \alpha$ of $H^{2}$ which leaves $l$ invariant. The previous paragraph shows that $\beta$ is the identity or a translation along $l$. Hence $\alpha$ is reflexion in $l$ or what is called a glide-reflexion along $l$.

At this point, we have developed all the basic facts about the geometry of the hyperbolic plane $H^{2}$. The same ideas can be used to develop the theory of hyperbolic $n$-space $H^{n}$ using inversions in spheres of dimension $(n-1)$.

We have just seen that there are three types of orientation preserving isometry of $H^{2}$, namely rotations, parabolics and hyperbolics, and these are characterised by the number of points left fixed on the circle at infinity, namely 0,1 or 2 . Note also that a rotation fixes one point of $\mathrm{H}^{2}$, and parabolics and hyperbolics fix no points of $\mathrm{H}^{2}$. We can use these facts to show that isometries of $H^{2}$ rarely commute, which is quite different from the Euclidean situation where any two translations commute. To be precise, we can prove the following. Identify $H^{2}$ with the open unit disc and if $\alpha$ is an isometry of $H^{2}$, let fix $(\alpha)$ denote the set of points in $H^{2} \cup S^{1}$ left fixed by $\alpha$.

LEMMA 1.10. (i) If $\alpha$ and $\beta$ are two non-trivial orientation preserving isometries of $H^{2}$, then $\alpha$ and $\beta$ commute if and only if $\operatorname{fix}(\alpha)=\operatorname{fix}(\beta)$.

(ii) If $\alpha$ is a non-trivial orientation preserving isometry of $\mathrm{H}^{2}$, then the group $C(\alpha)$ of all orientation preserving isometries of $H^{2}$ which commute with $\alpha$ is abelian and is isomorphic to $S^{1}$ if $\alpha$ is a rotation and isomorphic to $\mathbb{R}$ otherwise.

Remark. It follows at once that the torus $S^{1} \times S^{1}$ cannot admit a hyperbolic structure. For this would imply that $\mathbb{Z} \times \mathbb{Z}$ acts discretely and orientation preservingly on $H^{2}$ and hence that $\mathbb{Z} \times \mathbb{Z}$ is a discrete subgroup of $\mathbb{R}$ or $S^{1}$, either of which is impossible.

Proof of Lemma 1.10. The points fixed by $\beta \alpha \beta^{-1}$ are exactly the points of $\beta(\mathrm{fix}(\alpha))$. If $\alpha$ and $\beta$ commute, it follows that $\beta$ leaves fix $(\alpha)$ invariant.

If $\alpha$ is a rotation, fix $(\alpha)$ is a single point $P$ lying in $H^{2}$. If $\beta$ commutes with $\alpha$, it follows that $\beta$ must fix $P$ and hence is also a rotation about $P$. Hence $\operatorname{fix}(\alpha)=\operatorname{fix}(\beta)$. Further, $C(\alpha)$ consists of all rotations about $P$ and so is isomorphic to $S^{1}$.

If $\alpha$ is hyperbolic, fix $(\alpha)$ is two points $x$ and $y$ on $S^{1}$. If $\beta$ commutes with $\alpha$, it follows that $\beta$ must fix $x$ and $y$ or interchange them. But the only orientation preserving isometry of $H^{2}$ which can interchange two points of $S^{1}$ is a rotation 
through $\pi$, and $\beta$ cannot be a rotation by the previous paragraph. Hence fix $(\alpha)=$ fix $(\beta)$. Further, $C(\alpha)$ consists of all hyperbolic isometries of $H^{2}$ which fix $x$ and $y$ and so $C(\alpha)$ is isomorphic to $\mathbb{R}$.

If $\alpha$ is parabolic, fix $(\alpha)$ is a single point $x$ of $S^{1}$. As before, it follows that if $\beta$ commutes with $\alpha$, then $\beta$ must also fix $x$. Now the previous paragraph shows that if $\beta$ were hyperbolic then $\alpha$ would also be hyperbolic. Hence $\beta$ must be parabolic and so $\operatorname{fix}(\alpha)=\mathrm{fix}(\beta)$. Further, $C(\alpha)$ consists of all parabolic isometries of $H^{2}$ fixing $x$ and so is isomorphic to $\mathbb{B}$.

We close this section by discussing discrete groups of isometries of $H^{2}$. If $G$ is such a group and if $G$ acts freely on $H^{2}$, then, as usual, $H^{2} / G$ inherits a natural metric such that the projection $H^{2} \rightarrow H^{2} / G$ is a local isometry. If $G$ does not act freely, then $H^{2} / G$ still inherits a natural metric, but now there are singular points of three types--cone points, reflexion lines and corner reflectors. Again a good class of examples is the triangle groups. Let $p, q$ and $r$ be integers such that $\frac{1}{p}+\frac{1}{q}+\frac{1}{r}<1$. Then there is a triangle $\Delta$ in $H^{2}$, unique up to congruence, with angles $\pi / p, \pi / q$ and $\pi / r$. As usual, we define $\Delta^{*}(p, q, r)$ to be the group of isometries of $H^{2}$ generated by reflexions in the edges of $\Delta$ and define $\Delta(p, q, r)$ to be the orientation preserving subgroup. It can be proved that the translates of $\triangle$ by $\Delta^{*}(p, q, r)$ tile $H^{2}$ and that the stabilizer of $\triangle$ is trivial, but this is not the obvious fact it was in the previous cases. It follows that $H^{2} / \triangle^{*}(p, q, r)$ is isometric to the original triangle $\triangle$ and that $H^{2} / \triangle(p, q, r)$ is isometric to the double of $\triangle$ and so is a 2-sphere with hyperbolic metric except at three cone points.

One can define groups which act freely on $H^{2}$ by a similar method of starting with a fundamental region. For example, let $X$ be a regular octagon in $H^{2}$ with all angles $\pi / 4$ as shown in Fig. 1.11 and let $\alpha, \beta, \gamma$ and $\delta$ be the isometries of $H^{2}$ such that $\alpha(A B)=D C, \beta(B C)=E D, \gamma(E F)=H G, \delta(F G)=A H$ and, in all cases, the interior of $X$ is disjoint from its translates. Then the group of isometries $\Gamma$ of $H^{2}$ generated by $\alpha, \beta, \gamma$ and $\delta$ is discrete and has $X$ as a fundamental region. The quotient $H^{2} / \Gamma$ can be obtained from $X$ by identifying the pairs of edges identified by $\alpha, \beta, \gamma$ and $\delta$. This is a standard picture for the closed orientable surface of genus two, $T_{2}$. Note that the eight vertices of $X$ are identified to one point in $H^{2} / \Gamma$ which is, therefore, not a cone point as the angle round it is $2 \pi$. Thus $H^{2} \mid \Gamma$ has no singularities, so $\Gamma$ acts freely on $H^{2}$ and is isomorphic to $\pi_{1}\left(T_{2}\right)$.

The closed orientable surface of genus $g, T_{g}$, is a finite covering of $T_{2}$, so $\pi_{1}\left(T_{g}\right)$ is a subgroup of $\pi_{1}\left(T_{2}\right)$ and so it also acts freely on $H^{2}$ with quotient homeomorphic to

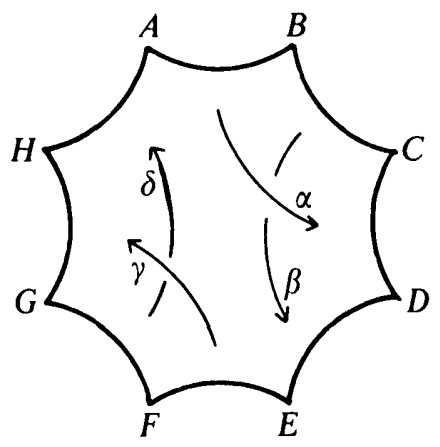

FIG. 1.11 
$T_{g}$. In the language of the introduction, every closed orientable surface of genus at least two admits a geometric structure modelled on $H^{2}$ or more briefly admits a hyperbolic structure. It is easy to do a similar construction to obtain a hyperbolic structure on the closed non-orientable surface with Euler number - 1. As every closed surface with negative Euler number covers this surface, we deduce that, as stated in the introduction, every closed surface of negative Euler characteristic admits a hyperbolic structure. This completes the result, also stated in the introduction, that every closed surface admits a geometric structure.

Now every simply connected surface with transitive isometry group is isometric to one of $S^{2}, E^{2}$ or $H^{2}$ after a scale change, e.g. one can obtain spheres of many different radii. Hence there are only three possible types of geometric structure on a surface. A final point is that a given closed surface $F$ can admit a geometric structure of only one of these three types. This is a trivial consequence of the Gauss-Bonnet Theorem, and we discuss the argument at the end of $\S 2$, but here is a more simpleminded proof.

If $F$ is $S^{2}$ or $P^{2}$, then the universal cover of $F$ is $S^{2}$ so that $F$ cannot admit a Euclidean or hyperbolic structure. Any discrete group of isometries of $E^{2}$ with compact quotient has a subgroup of finite index isomorphic to $\mathbb{Z} \times \mathbb{Z}$. Hence if $F$ admits a Euclidean structure, $F$ must be finitely covered by the torus, so that $F$ must be $T$ or the Klein bottle $K$. Thus surfaces of negative Euler number cannot admit a Euclidean structure. Finally, the torus $T$ cannot admit a hyperbolic structure by the Remark following the statement of Lemma 1.10.

The last few paragraphs showed that each closed surface admits a geometric structure and that the geometry involved is unique. When one considers non-closed surfaces, the situation is similar. Recall that, by definition, a geometric structure on a manifold with boundary is just a geometric structure on the interior of the manifold, so we can restrict our discussion to the case of surfaces without boundary. A noncompact manifold without boundary is called open. It can be shown that every open surface admits a hyperbolic structure so we have the nice situation that every surface admits a geometric structure. However, one does not quite get the uniqueness of the geometry involved. Of course, no open surface can be modelled on $S^{2}$, as it cannot be covered by a compact surface. But exactly three open surfaces, namely the plane, cylinder and Moebius band, can be modelled on $E^{2}$.

Note that these comments about geometric structures on open surfaces use the fact that we only allow complete metrics. This is why a surface with a geometric structure modelled on $S^{2}, E^{2}$ or $H^{2}$ must have universal covering isometric to $S^{2}, E^{2}$ or $H^{2}$. As an example, the plane admits an incomplete metric of constant positive curvature obtained by identifying the plane with some open subset of $S^{2}$, for example $S^{2}$ with one point removed. This is an example of a surface with an incomplete geometric structure modelled on $S^{2}$ and, of course, its universal covering is not $S^{2}$. See Thurston [66] for a discussion of incomplete geometric structures.

\section{§2. Geometric structures on 2-dimensional orbifolds}

If $G$ is a discrete group of isometries of a Riemannian manifold $M$ (any dimension), and if $G$ acts freely on $M$, then the quotient space with the natural metric is also a Riemannian manifold. In $\$ 1$, we saw that it is very natural to consider also discrete groups of isometries which do not act freely. In this case, the quotient space 
still has a natural metric but is no longer a Riemannian manifold. In fact, the quotient space need not be topologically a manifold. For example if the group $\mathbb{Z}_{2}$ acts on $E^{3}$ by the map $x \mapsto-x$, then the quotient space is homeomorphic to a cone on $P^{2}$ and this fails to be a manifold at the cone point. We want to introduce some terminology to describe the kind of spaces one can obtain in this way. We first introduce the idea of an abstract space with singularities and without a metric and then consider possible metrics afterwards, by analogy with the way in which one introduces manifolds and then considers Riemannian manifolds.

Recall that a $n$-manifold (without boundary) is a Hausdorff, paracompact space which is locally homeomorphic to $\mathbb{R}^{n}$. Also a smooth $n$-manifold comes supplied with an atlas of charts whose overlap is smooth. Following Thurston [66], one defines a $n$-dimensional orbifold (without boundary) to be a Hausdorff, paracompact space which is locally homeomorphic to the quotient space of $\mathbb{R}^{n}$ by a finite group action. More precisely, the given space is equipped with a covering by open sets $\left\{U_{i}\right\}$ closed under finite intersections. To each $U_{i}$ is associated a finite group $\Gamma_{i}$ and an action of $\Gamma_{i}$ on an open subset $\hat{U}_{i}$ of $\mathbb{R}^{n}$ and a homeomorphism $\phi_{i}: \hat{U}_{i} / \Gamma_{i} \rightarrow U_{i}$. Whenever $U_{i} \subset U_{j}$, there is to be an inclusion $f_{i j}: \Gamma_{i} \rightarrow \Gamma_{j}$ and an embedding $\bar{\phi}_{i j}: \tilde{U}_{i} \rightarrow \tilde{U}_{j}$ equivariant with respect to $f_{i j}$ such that the following diagram commutes.

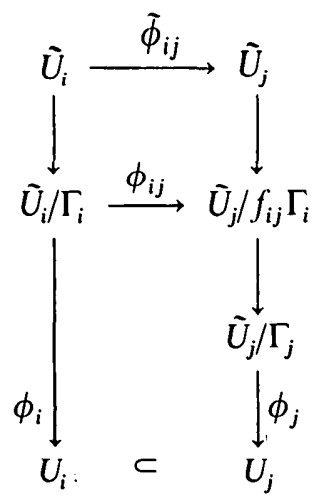

Of course, the covering $\left\{U_{i}\right\}$ is not, in general, an intrinsic part of the structure of an orbifold, but it will be so if it is maximal among coverings satisfying the above conditions. An isomorphism of orbifolds is a homeomorphism which respects the given quotient structures on open subsets of the orbifolds. One can define smooth orbifolds similarly, by insisting that $\Gamma_{i}$ act smoothly on $\tilde{U}_{i}$ and that $\tilde{\phi}_{i j}$ be smooth.

It is easy to see that if a group $G$ acts properly discontinuously on a smooth manifold $M$, then the quotient space is a smooth orbifold. The example in the first paragraph of this section shows that, in general, an orbifold is not even homeomorphic to a manifold. However, in dimension two, any orbifold is homeomorphic to a manifold, because the only possible types of singular point are cone points, reflector lines and corner reflectors. (Note that the idea of a cone point is perfectly well defined without having a metric.) It is important to realize that an orbifold with cone points is homeomorphic to a manifold but is not isomorphic to one. So one must distinguish carefully between a 2-dimensional orbifold and the underlying surface. Note also that if an orbifold has reflexion curves, the points on these curves are not boundary points of the orbifold. In fact so far we have only discussed orbifolds without boundary. We may need to discuss orbifolds with 
boundary, and they are defined as spaces locally homeomorphic to the quotient of $\mathbb{R}_{+}^{n}$ by a finite group action.

It is not difficult to define the idea of a metric on an orbifold and hence the idea of a geometric structure. But we will not need to do this. For the question of whether a 2-dimensional orbifold $M$ admits a geometric structure is equivalent to asking whether $M$ is isomorphic (as orbifold) to the quotient of $S^{2}, E^{2}$ or $H^{2}$ by some discrete group of isometries. Before answering this question we need to introduce the concepts of an orbifold covering space and of the fundamental group of an orbifold.

Recall that a continuous map $f: X \rightarrow Y$ is a covering if any point $y$ in $Y$ has a neighbourhood $U$ such that $f^{-1}(U)$ is the disjoint union of sets $V_{\lambda}, \lambda \in \Lambda$, such that $f \mid: V_{i} \rightarrow U$ is a homeomorphism. If $X$ and $Y$ are orbifolds and $f: X \rightarrow Y$ is an orbifold map, one defines an orbifold covering in the same way except that one allows $f \mid: V_{i} \rightarrow U$ to be the natural quotient map between two quotients of $\mathbb{R}^{n}$ by finite groups, one of which is a subgroup of the other. Thus if a group $G$ acts properly discontinuously on diffeomorphisms of a smooth manifold $M$ and if $H$ is a subgroup of $G$, then the natural map $M / H \rightarrow M / G$ is an orbifold covering. In particular, the projection $M \rightarrow M / G$ is an orbifold covering. Note that an orbifold covering need not be a covering map of the underlying spaces. In fact, the underlying space of $M / G$ can be simply connected as in the case of the quotient of $S^{2}, E^{2}$ or $H^{2}$ by a triangle group.

It is easy to define a universal covering orbifold of an orbifold in the same way as one defines universal covering spaces and the same uniqueness holds. There are general arguments for showing that any orbifold has a universal covering orbifold, but I will simply give a proof in dimension two.

Let $M$ be a 2 -dimensional orbifold without boundary. The underlying surface may have boundary but in this case the double of $M$ along its boundary is a 2-fold orbifold covering of $M$ whose underlying surface has no boundary. Thus any 2-dimensional orbifold is orbifold covered by an orbifold whose only singularities are cone points, and it suffices to show that such an orbifold has a universal covering. Let $X$ denote such an orbifold and let $\Sigma$ denote the union of the cone points of $X$. If $\tilde{X}$ is an orbifold covering of $X$ with projection map $p$ then $\tilde{X}-p^{-1}(\Sigma)$ is a covering, in the usual sense, of $X-\Sigma$. Further, if $x_{i}$ is a cone point of $X$ with cone angle $2 \pi / n_{i}$ (i.e. a neighbourhood of $x_{i}$ is isomorphic to $\mathbb{R}^{2} / \mathbb{Z}_{n_{i}}$ with $x_{i}$ corresponding to the cone point) then any cone point in $\tilde{X}$ lying above $x_{i}$ has cone angle $2 \pi / m_{i}$ where $m_{i} \mid n_{i}$. Let $N$ denote $X$ with the interiors of small cones centred on the cone points removed and let $\hat{N}$ denote $p^{-1}(N)$ in $\hat{X}$. Thus $X-\Sigma$ is simply $N$ with open collars attached to $\partial N$. Then the covering $\bar{N} \rightarrow N$ induced by the orbifold covering $\hat{X} \rightarrow X$ has the property that a circle $C_{i}$ of $\partial N$ bounding a cone with angle $2 \pi / n_{i}$ has pre-image consisting of circles which must project with degree which divides $n_{i}$. Thus $\pi_{i}(\bar{N})$ must contain all conjugates of $\alpha_{i}^{n_{i}}$ where $\alpha_{i}$ is any element of $\pi_{1}(N)$ represented by $C_{i}$.

We define a quotient group $G$ of $\pi_{1}(N)$ by adding the relations $\alpha_{i}^{n_{i}}=1$. Clearly $\pi_{1}(\bar{N})$ contains the kernel $K$ of the natural homomorphism $\pi_{1}(N) \rightarrow G$. It follows that the covering $N_{1}$ of $N$ determined by $K$ is universal among all coverings which extend to orbifold coverings of $X$ and so $N_{1}$ with appropriate cones added to $\partial N_{1}$ is a universal orbifold covering of $X$. As with coverings in the ordinary sense, $X$ is the quotient of this universal covering by the action of a group, namely $G$. Hence, by analogy with this case, one calls $G$ the orbifold fundamental group of $X$.

Note that during the above argument we obtained a presentation of the fundamental group of an orbifold $X$ with no reflector curves. One starts with $\pi_{1}(N)$, 
where $N$ is $X$ with a neighbourhood of the cone points removed and one adds the relations $\alpha_{i}^{n_{i}}=1$. Thus, if the underlying surface of $X$ is the closed orientable surface of genus $g$ and if $X$ has $n$ cone points with cone angle $2 \pi / p_{i}, 1 \leqslant i \leqslant n$, then a presentation for $\pi_{1}(X)$ is

$$
\left\{a_{1}, b_{1}, \ldots, a_{g}, b_{g}, x_{1}, \ldots, x_{n}: x_{i}^{p_{i}}=1, \prod_{i=1}^{g}\left[a_{i}, b_{i}\right] x_{i} \ldots x_{n}=1\right\} .
$$

One proceeds in a similar way to find presentations of orbifold fundamental groups in general. The nicest way to think about it is to let $U$ be a regular neighbourhood of the singular set of $X$ and let $N$ be the closure of $X-U$. As $N$ is a surface, its fundamental group is free and is no problem. The components of $U$ are of three types. Firstly, one can have a cone that is the quotient of $D^{2}$ by a finite cyclic group of rotations. The orbifold fundamental group of a cone is, of course, finite cyclic. Secondly, one can have an orbifold whose underlying space is $\mathbb{R} \times I$ and which has one reflector line and one boundary line. This orbifold has fundamental group $\mathbb{Z}_{2}$ and its universal covering orbifold is the manifold $\mathbb{R} \times I$. Finally, one can have an orbifold whose underlying space is $S^{1} \times I$ and which has one reflector circle and one boundary circle. The fundamental group of this orbifold is $\mathbb{Z} \times \mathbb{Z}_{2}$ and its universal covering orbifold is the manifold $\mathbb{R} \times I$ again. Now $X$ is the union of the surface $N$ with the orbifolds which are the components of $U$ and so $\pi_{1}(X)$ can be calculated using what is effectively van Kampen's Theorem. The case previously discussed, where $X$ has no reflector curves, is a special case of this approach. As an example, if the orbifold $X$ has underlying surface $Y$ equal to a torus with the interior of a 2-disc removed and has $\partial Y$ as a reflexion circle, then $\pi_{1}(X)$ is an amalgamated free product of the form $\pi_{1}(Y) *_{Z}\left(\mathbb{Z} \times \mathbb{Z}_{2}\right)$. See Fig. 2.1. In terms of generators and relations one starts with $\pi_{1}(Y)$ and adds a new generator $u$ such that $u^{2}=1$ and $u^{-1} x u=x$, where $x$ represents the boundary circle of $Y$. If $X$ is an orbifold with underlying space $Y$ and a reflector line in $\partial Y$, then $\pi_{1}(X)$ is isomorphic to $\pi_{1}(Y) * \mathbb{Z}_{2}$.

So far, the only examples of orbifolds which we have seen are orbifolds covered by manifolds. However, not every orbifold has this property. The teardrop orbifold, see Fig. 2.2, which has underlying surface $S^{2}$ and has a single cone point with angle $2 \pi / n, n \geqslant 2$, has no orbifold coverings at all, so its orbifold fundamental group is trivial. This is because $S^{2}$ minus a point is simply connected (in the ordinary sense) and so has no coverings.

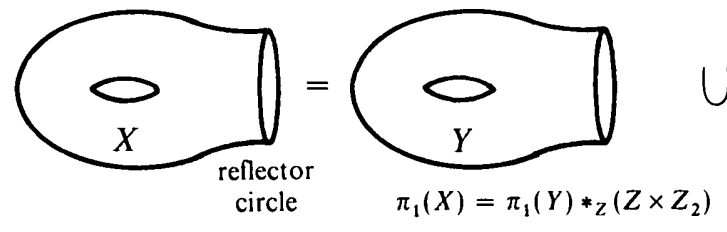

FIG. 2.1

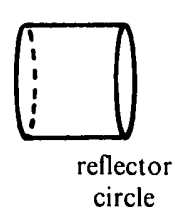

circle

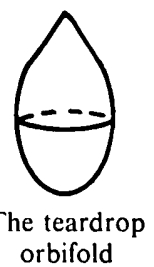

FIG. 2.2

This also follows directly from the presentation given above for the fundamental group of a 2-dimensional orbifold. The orbifold fundamental group of the teardrop is obtained from the fundamental group of $S^{2}$ minus a point by adding a relation, so is obviously trivial.

An orbifold such as the teardrop is called bad. An orbifold is good if it is orbifold covered by some manifold or, equivalently, if its universal covering orbifold is a manifold. As surfaces are completely classified, it is clear how to describe all 
2-dimensional orbifolds and, in particular, it is easy to decide precisely which orbifolds are bad.

THEOREM 2.3. The only bad 2-dimensional orbifolds without boundary are of the following types

Underlying surface

Cone points
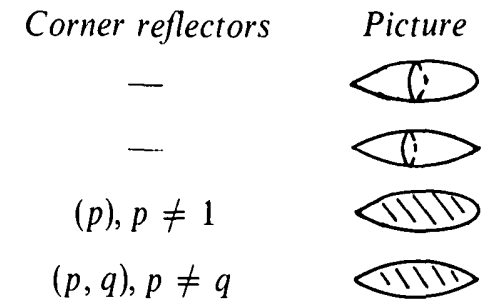

The integers in brackets denote the angles at the singular points. For a cone point, $(p)$ denotes an angle $2 \pi / p$. For a corner reflector, $(p)$ denotes angle $\pi / p$.

Proof. Let $X$ be a bad 2-dimensional orbifold without boundary and with no proper orbifold covering. We will show that $X$ must be one of (i) or (ii). Any orbifold covered by $X$ must be one of the types listed, so the result will follow.

First recall the example of an orbifold with underlying surface $S^{2}$ and three cone points with cone angles $\pi / p, \pi / q, \pi / r$. From $\S 1$, we know that this orbifold is the quotient of $S^{2}, E^{2}$ or $H^{2}$, as appropriate, by the triangle group $\Delta(p, q, r)$. In particular, such an orbifold has a proper orbifold covering. It follows that any orbifold $Y$ with three or more cone points has a proper covering. For, in the underlying surface of $Y$ there is a 2-disc $D$ which contains three cone points and one can construct a proper orbifold covering $\bar{Y}$ of $Y$ by taking a proper orbifold covering $\bar{D}$ of $D$ (which is an orbifold with boundary), such that each boundary component of $\tilde{D}$ projects homeomorphically to $\partial D$, and glueing a copy of $Y-D$ to each boundary component of $\bar{D}$.

It is also clear that an orbifold $X$ with reflector lines has a proper covering, namely the orbifold obtained by doubling $X$ along the reflector lines. (One takes two disjoint copies of $X$ and glues the reflector lines of one copy to those of the other by the identity map.) Finally, if the underlying surface $F$ of an orbifold $X$ is not simply connected, then $F$ has a proper covering $\hat{F}$, and $\hat{F}$ inherits a natural orbifold structure $\bar{X}$ so that $\bar{X}$ is a proper orbifold covering of $X$.

Thus a 2-dimensional orbifold without boundary which has no proper coverings must have underlying surface $S^{2}$ or $\mathbb{R}^{2}$ and have at most two cone points. But the fundamental group of an orbifold $X$ with underlying surface $\mathbb{R}^{2}$ is a free product of cyclic groups, one for each cone point. Hence if $X$ has no proper coverings, it must have no cone points. It follows that if $X$ is bad and has no proper orbifold coverings, then its underlying surface must be $S^{2}$, so that we have cases (i) or (ii) of the theorem as stated.

Now we come to the result about geometric structures on 2-dimensional orbifolds. This is the best possible result. See [66] for a proof.

THEOREM 2.4. Every good 2-dimensional orbifold without boundary admits a geometric structure modelled on $\mathrm{S}^{2}, E^{2}$ or $\mathrm{H}^{2}$, that is, it is isomorphic as orbifold to the quotient of $\mathrm{S}^{2}, E^{2}$ or $\mathrm{H}^{2}$ by some discrete group of isometries. 
This result gives us new information about surfaces. In particular if $F$ is a surface and $G$ is a finite group acting on $F$, then the quotient space is an orbifold $X$. This is a good orbifold as $X$ is orbifold covered by the manifold $F$. Now the existence of a geometric structure on $X$ is equivalent to asserting that there is a constant curvature metric on $F$ such that $G$ is a group of isometries of $F$ with respect to this metric.

A question naturally raised by the above comments is which 2-dimensional orbifolds are finitely covered by a manifold. The answer is again the best possible.

THEOREM 2.5. Every good, compact 2-dimensional orbifold without boundary is finitely covered by a manifold.

This theorem is equivalent to asserting that any finitely generated, discrete group $\Gamma$ of isometries of $S^{2}, E^{2}$ or $H^{2}$ with compact quotient space has a torsion free subgroup of finite index. This fact is trivial for $S^{2}$, and easy for $E^{2}$. The first proof for $H^{2}$ was completed by Fox [17]. See also [6]. A less elementary but more attractive proof is to quote the theorem of Selberg [61] that any finitely generated matrix group with entries in a field is residually finite. Recall that $\Gamma$ is a subgroup of $\operatorname{PSL}(2, \mathbb{R})$, and so $\Gamma$ is residually finite. One then obtains a torsion free subgroup of finite index by avoiding the finitely many conjugacy classes of torsion in $\Gamma$. (These conjugacy classes are finite in number as they correspond to the cone points, corner reflectors and reflector lines in $H^{2} / \Gamma$.)

We have just seen that discrete groups of isometries of $S^{2}, E^{2}$ or $H^{2}$ with compact quotient all contain torsion free subgroups of finite index. Such a torsion free subgroup is the fundamental group of some closed surface. There is a converse to this result whose proof was recently completed by Kerckhoff [27].

THEOREM 2.6. Let $M$ be a closed surface not $S^{2}$ or $P^{2}$ and suppose that $\pi_{1}(M)$ is a normal subgroup of a group $G$ with finite quotient $K$. Suppose further that the natural homomorphism $K \rightarrow$ Out $\pi_{1}(M)$ is injective. Then $G$ is isomorphic to a discrete group of isometries of $E^{2}$ or $H^{2}$.

If $M$ is a Euclidean surface, then it is standard that the hypotheses imply that $G$ is a crystallographic group. See Wolf [75]. If $M$ is hyperbolic, various special cases of the theorem have been known for some time (see [45, 13, 33, 78, 76, 77, 8 and 79]), but Kerckhoff's paper [27] is the only one to handle this case in full generality.

I want to end this section by introducing the Euler number of a compact orbifold. First recall that any finite simplicial complex $K$ has an Euler number $\chi(K)$, and if $\alpha_{i}$ denotes the number of $i$-simplexes in $K$, then $\chi(K)=\sum_{i \geqslant 0}(-1)^{i} \alpha_{i}$. This number is a topological invariant because it can be shown to equal $\sum_{i \geqslant 0}(-1)^{i} \beta_{i}$, where $\beta_{i}$ denotes the rank of $H_{i}(K, \mathbb{Z})$. The definition makes it obvious that if $\tilde{K}$ is a $n$-fold covering space of $K$ then $\chi(\tilde{K})=n \cdot \chi(K)$.

Now every good compact 2-dimensional orbifold $X$ is finitely covered by a surface $\bar{X}$, so it is natural to define the Euler number of $X$ by the equation $\chi(\tilde{X})=n \cdot \chi(X)$, where $n$ is the degree of the covering map $\tilde{X} \rightarrow X$. Note that $\chi(X)$ will not, in general, be an integer, but is always rational. We can compute $\chi(X)$ directly from a description of the orbifold $X$. 
Suppose that $X$ is a good compact 2-dimensional orbifold with underlying surface $Y$ and $m$ cone points with cone angles $2 \pi / q_{i}, 1 \leqslant i \leqslant m$, and no reflector curves. Let $D_{1}, \ldots, D_{m}$ be disjoint 2-discs in $Y$ each containing one of the cone points and let $W$ denote the closure of the complement of the union of the discs $D_{i}$. Thus $Y=W \cup\left(\bigcup_{i=1}^{m} D_{i}\right)$. As $\chi\left(D_{i}\right)=1$ and $\chi\left(S^{1}\right)=0$, we obtain the equation $\chi(Y)=\chi(W)+m$. As $X$ is good, it has a finite orbifold covering $\bar{X}$ with no singularities. Let $\tilde{W}$ denote the pre-image in $\tilde{X}$ of $W$. The projection $\tilde{W} \rightarrow W$ is a covering map (in the ordinary sense) of some degree $d$. Hence $\chi(\tilde{W})=d \cdot \chi(W)$. Now the pre-image in $\hat{X}$ of $D_{i}$ is only $d / q_{i}$ 2-discs, so we obtain

$$
\chi(\tilde{X})=d \cdot \chi(W)+\sum_{i=1}^{m} d / q_{i}
$$

By definition, $\chi(\tilde{X})=d \cdot \chi(X)$, so by dividing the equation by $d$ and substituting $\chi(W)=\chi(X)-m$, we obtain

$$
\chi(X)=\chi(Y)-\sum_{i=1}^{m}\left(1-\frac{1}{q_{i}}\right)
$$

This is often known as the Riemann-Hurwitz formula.

Now suppose that $X$ is a good compact 2-dimensional orbifold with underlying surface $Y$ and $m$ cone points with cone angle $2 \pi / q_{i}, 1 \leqslant i \leqslant m$, and $n$ corner reflectors with angle $\pi / r_{j}, 1 \leqslant j \leqslant n$. Let $D X$ denote the orbifold obtained by doubling $X$ along all reflector curves, and denote its underlying surface by $D Y$. Then $D X$ is naturally a 2-fold orbifold covering of $X$ and $D X$ has $m$ pairs of cone points with cone angle $2 \pi / q_{i}, 1 \leqslant i \leqslant m$, and $n$ cone points with angle $2 \pi / r_{j}, 1 \leqslant j \leqslant n$. The preceding paragraph shows that

$$
\chi(D X)=\chi(D Y)-2 \sum_{i=1}^{m}\left(1-\frac{1}{q_{i}}\right)-\sum_{j=1}^{n}\left(1-\frac{1}{r_{j}}\right)
$$

and as $\chi(D X)=2 \chi(X)$ and $\chi(D Y)=2 \chi(Y)$, we obtain

$$
\chi(X)=\chi(Y)-\sum_{i=1}^{m}\left(1-\frac{1}{q_{i}}\right)-\frac{1}{2} \sum_{j=1}^{n}\left(1-\frac{1}{r_{j}}\right)
$$

This is the most general version of the Riemann-Hurwitz formula.

This formula can now be used to define the Euler number of a bad orbifold also. Thus the teardrop orbifold $S^{2}(p)$ has Euler number $2-\left(1-\frac{1}{p}\right)=1+\frac{1}{p}$. This formula makes it clear, yet again, that the orbifold cannot be covered by a manifold or indeed by any other orbifold. For the Euler number of a compact 2-dimensional orbifold is always less than or equal to 2 .

So far, the approach to the definition of the Euler number of an orbifold has been very combinatorial, but there is a beautiful connection between the preceding discussion and the geometry of the situation which is provided by the Gauss-Bonnet Theorem. First, we discuss this connection in the context of surfaces.

Let $F$ be a closed surface with some Riemannian metric. This metric will provide $F$ with a Gauss curvature function $K$ describing the curvature of $F$ at each point, and 
an area element $d A$. The Gauss-Bonnet Theorem asserts that for any closed surface with any Riemannian metric, the integral $\int_{F} K d A$ must equal $2 \pi \gamma(F)$. Suppose we specialise to the case of a metric on $F$ with constant curvature $K_{0}$. Then the integral becomes $K_{0} \cdot A(F)$ where $A(F)$ denotes the area of $F$ in the given metric. It follows trivially that $K_{0}$ and $\chi(F)$ must have the same sign, thus providing the proof promised at the end of $\S 1$, that a closed surface admits a geometric structure of only one of the three possible types. Note also, that it follows that if $\chi(F) \neq 0$, then all constant curvature metrics on $F$, with curvature 1 or -1 , yield the same area-a very surprising fact.

Now consider a good compact orbifold $X$ which is expressed as $S^{2} / \Gamma, E^{2} / \Gamma$ or $H^{2} / \Gamma$ for some discrete group $\Gamma$ of isometries of $S^{2}$ or $H^{2}$ and has the induced metric. As discussed in $\$ 1, \quad X$ has a well defined area $A(X)$ which has the same naturality property under finite coverings as the Euler number, i.e. if $\bar{X}$ is an orbifold covering of $X$ of degree $n$, then $A(\bar{X})=n \cdot A(X)$. Now we can use the fact that $X$ is finitely orbifold covered by some surface $F$ and apply the Gauss-Bonnet Theorem to $F$. If $X$ is $S^{2} / \Gamma$, we deduce that $A(X)=2 \pi \psi(X)$ and if $X$ is $H^{2} / \Gamma$, we deduce that $A(X)=-2 \pi \gamma(X)$.

An interesting example is the case when $\Gamma$ is a triangle group $\triangle^{*}(p, q, r)$, so that $X$ is just a triangle with angles $\pi / p, \pi / q, \pi / r$. Recall that a triangle on $S^{2}$ with angles $\alpha, \beta$ and $\gamma$ has area $\alpha+\beta+\gamma-\pi$ and that a triangle in $H^{2}$ with angles $\alpha, \beta$ and $\gamma$ has area $\pi-\alpha-\beta-\gamma$. We can now see that it is no coincidence that these formulas are the same apart from sign change. The above discussion gives a new derivation of these formulas (at least when the angles are of the form $\pi / p, \pi / q, \pi / r$ ), which makes it clear that the connection between them is due to the curvature of $H^{2}$ being equal but opposite in sign to that of $S^{2}$. There are versions of the Gauss-Bonnet Theorem for bounded surfaces which will do the same job for general triangles.

\section{§3. The basic theory of Seifert fibre spaces}

Seifert fibre spaces were defined and classified by Seifert [59]. W. Heil has translated this beautiful article into English and it can be found in [60]. Some other basic references on Seifert fibre spaces are Orlik's book [46], and $[16,44,48,47,51$, $72,43,73$ and 62 ]. See also [5] for a discussion of 3-dimensional orbifolds which are Seifert fibred.

A Seifert fibre space is a 3-manifold which is expressed as a union of disjoint circles, called fibres, in a particular way. The definition I am going to give is a little more general than Seifert's original definition.

We start by defining a trivial fibred solid torus as $S^{1} \times D^{\dot{2}}$ with the product foliation by circles. Thus the fibres are the circles $S^{1} \times\{y\}$, for $y$ in $D^{2}$. A fibred solid torus is a solid torus with a foliation by circles which is finitely covered by a trivial fibred solid torus. Such an object can be constructed from a trivial fibred solid torus by cutting it open along $\{x\} \times D^{2}$, for some $x$ in $S^{1}$, rotating one of the discs so obtained through $q / p$ of a full turn and glueing back together. This fibred solid torus will be $p$-fold covered by a trivial fibred solid torus. A fibred solid Klein bottle is a solid Klein bottle which is finitely covered by a trivial fibred solid torus. Such an object can be constructed from a trivial fibred solid torus by cutting it open along 
$\{x\} \times D^{2}$, for some $x$ in $S^{1}$, and glueing the two discs back together by a reflexion. As all reflexions of a disc are conjugate, we see that there is only one fibred solid Klein bottle (up to fibred homeomorphism) and this is double covered by a trivial fibred solid torus.

We can now define a Seifert fibre space to be a 3-manifold $M$ with a decomposition of $M$ into disjoint circles, called fibres, such that each circle has a neighbourhood in $M$ which is a union of fibres and is isomorphic to a fibred solid torus or Klein bottle. Clearly any circle bundle over a surface is a Seifert fibre space. Seifert's original definition [59] excluded the fibred solid Klein bottle as have most other writers on the subject. (But see [47 and 16].) We shall see that there are advantages in allowing such a phenomenon. Note that it does follow from this definition that a Seifert fibre space is foliated by circles. In [11], Epstein proved that the converse statement holds for compact 3-manifolds i.e. any foliation by circles of a compact 3-manifold is a Seifert fibration. Hence, for compact 3-manifolds, a manifold is a Seifert fibre space if and only if it is foliated by circles. Of course, this simple statement would be false if one kept to Seifert's original definition of a Seifert fibre space. Note that a special case of Epstein's result is that the only foliations by circles of the solid torus and solid Klein bottle are those described in the previous paragraph.

Now we can consider some elementary facts about Seifert fibre spaces. I have already pointed out that all fibred solid Klein bottles are isomorphic. If a fibred solid torus $T(p, q)$ is constructed from a trivial such by cutting open along a 2-disc and glueing with $q / p$ of a full twist, then clearly all the fibres in $T$ apart from the central one represent $p$ times the generator of $\pi_{1}(T)$ and they also wind $q$ times around the central fibre. (This assumes that $p$ and $q$ are co-prime.) Hence if $T(p, q)$ and $T\left(p^{\prime}, q^{\prime}\right)$ are isomorphic, it follows that $p=p^{\prime}$ and $q \equiv \pm q^{\prime}(\bmod p)$. Note that one can alter $q$ by an integral multiple of $p$ by cutting $T(p, q)$ along a 2-disc and glueing back with a full twist. Hence $p$ is an invariant of a fibred solid torus and $q$ will also be an invariant if we normalize $q$ so that $0 \leqslant q \leqslant \frac{1}{2} p$.

If we orient the solid tori and are careful about other orientations, we need only normalise $q$ so $0 \leqslant q<p$ in order to obtain an invariant of oriented fibred solid tori. The invariants $(p, q)$, normalised appropriately, are called the orbit invariants of the central fibre. We will use the following orientation conventions which seem to be standard [46]. There is no strong reason for the particular convention chosen. Firstly, given an oriented manifold $M$, its boundary $\partial M$ is given the orientation which followed by an inward normal coincides with the orientation of $M$. Secondly, if $M$ is an oriented solid torus, we choose orientations for the $D^{2}$ and $S^{1}$ factors so that they give the orientation of $M$. Notice that the orientation of the factors can be taken in either order. Finally, we need to specify that the standard oriented fibred solid torus $T(p, q)$ is constructed from the standard oriented trivial fibred solid torus $S^{1} \times D^{2}$ by using an anti-clockwise twist through $2 \pi q / p$ on the $D^{2}$-factor. Notice that reversing the orientations of both factors of $S^{1} \times D^{2}$ yields an object which is orientation preservingly isomorphic to $T(p, q)$.

It now makes sense to call a fibre regular if it has a neighbourhood isomorphic to a trivial fibred solid torus and critical otherwise. A fibred solid torus has at most one critical fibre - namely the centre fibre. However, a fibred solid Klein bottle $K$ has a continuous family of critical fibres whose union forms an annulus. If $K$ is constructed from a trivial fibred solid torus $T$ by cutting $T$ along a 2-disc $D$ and glueing back using a reflexion of $D$ in the line $l$, then the union of the critical fibres is precisely 
$S^{1} \times l$. Hence in a general Seifert fibre space, the critical fibres are isolated or form subsurfaces.

The reason for the terminology 'fibre' is that one can think of a Seifert fibre space $M$ as a kind of bundle in which the circles of our foliation of $M$ are the fibres. To construct the base space of this bundle we simply define $X$ to be the quotient space of $M$ obtained by identifying each circle to a point. If $M$ is a trivial fibred solid torus, then $X$ is clearly a 2-disc and the projection $M \rightarrow X$ is a bundle map in the usual sense. If $M$ is a fibred solid torus $T(p, q)$ then $M$ is $p$-fold covered by a trivial fibred solid torus $S^{1} \times D^{2}$. The corresponding action of $\mathbb{Z}_{p}$ on $S^{1} \times D^{2}$ is generated by a homeomorphism which is simply the product of a rotation through $2 \pi / p$ on the $S^{1}$-factor with a rotation through $2 \pi q / p$ on the $D^{2}$-factor. Notice that this action of $\mathbb{Z}_{p}$ on $S^{1} \times D^{2}$ obviously induces an action of $\mathbb{Z}_{p}$ on the base space $D^{2}$ which is generated by a rotation through $2 \pi q / p$. It follows that the space $X$ obtained from $M$ by identifying fibres to a point can be naturally identified with the quotient of $D^{2}$ by this action of $\mathbb{Z}_{p}$, that is, a cone orbifold with cone angle $2 \pi / p$. The projection $M \rightarrow X$ is not a bundle map in the usual sense, but we will think of it as a bundle map in a generalized sense. One can define the idea of a bundle in the category of orbifolds, but there seems no need to do that here. Precise definitions can be found in [46] and [66]. We shall call this generalized kind of circle bundle a Seifert bundle.

If $M$ is a fibred solid Klein bottle, then $M$ is double covered by a trivial fibred solid torus $S^{1} \times D^{2}$. The corresponding action of $\mathbb{Z}_{2}$ on $S^{1} \times D^{2}$ is generated by a homeomorphism which is simply the product of a rotation through $\pi$ on the $S^{1}$-factor with a reflexion of the $D^{2}$-factor. Clearly the space $X$ obtained from $M$ by identifying fibres to a point can be naturally identified with the quotient of $D^{2}$ by this action of $\mathbb{Z}_{2}$, which is an orbifold with boundary.

It follows from the above that the quotient space $X$ of a Seifert fibre space $M$ obtained by identifying each fibre to a point is (topologically speaking) a surface and naturally has an orbifold structure in which cone points correspond to orientation preserving critical fibres and points on reflexion curves correspond to orientation reversing critical fibres. This orbifold will have no corner reflectors. This last fact follows from the previous discussion. If one considers bundles over orbifolds in general then any orbifold can occur as the base, but we are considering only the situation where the total space is a manifold (i.e. an orbifold without singularities) and this is why corner reflectors cannot occur.

Note that if $M$ is a manifold without boundary, then $X$ is an orbifold without boundary, as reflector curves do not form part of the boundary of an orbifold. In general, $\partial M$ is simply the pre-image of $\partial X$ under the projection $M \rightarrow X$. Note that $\partial M$ is a union of tori and Klein bottles when $M$ is compact. In general, $\partial M$ can also contain open annuli and Moebius bands.

The base space $X$ of a Seifert fibre space $M$ is often called the orbit space of $M$. This terminology arises from the fact that it is often true that $X$ is the quotient of $M$ by a $S^{1}$-action. However, it is also often false that $X$ can be obtained this way, so I use the term base space to avoid being misleading as well as to fit with standard bundle terminology. A fibred solid Klein bottle admits a $S^{1}$-action leaving the fibration invariant and a fibred solid torus also admits such a $S^{1}$-action. Using these facts, one can show easily that if $M$ is a Seifert fibre space, then $M$ admits a $S^{1}$-action leaving the fibration invariant if and only if one can coherently orient all the fibres. It is also easy to see that any 3-manifold $M$ which admits a $S^{1}$-action without fixed points is a Seifert fibre space. The fibres of $M$ are simply the orbits of the $S^{1}$-action. All 
$S^{1}$-actions on 3-manifolds have been classified by Raymond [51], including those actions with fixed points.

The basic properties of bundles all extend to the category of bundles over orbifolds. In particular, there is an idea of induced bundle. Again, we will only need a special case of this construction so will discuss it in a more concrete way in the context of covering spaces.

We have seen already that a critical fibre of a Seifert fibre space $M$ is either isolated or is a fibre in a fibred solid Klein bottle $K$. In the second case, the union of critical fibres in $K$ forms a one-sided annulus in $K$. It follows that the union of all critical fibres in a Seifert fibre space $M$ consists of isolated fibres together with onesided annuli, tori or Klein bottles. Hence the union of the regular fibres in any connected Seifert fibre space $M$ is connected, and forms a bundle (in the usual sense). In particular, all the regular fibres of $M$ are freely homotopic to each other and any critical fibre has a power which is freely homotopic to a regular fibre. It follows that in a covering $\bar{M}$ of $M$, the foliation of $M$ by circles gives rise to a foliation of $\bar{M}$ by circles (which must again be a Seifert fibration) or to a foliation of $\bar{M}$ by lines. In either case, the base space of $\tilde{M}$, defined by identifying each leaf of the foliation to a point is an orbifold covering of the base space of $M$. This is obvious if $M$ is a fibred solid torus or Klein bottle and it then follows for any Seifert fibre space. Conversely, if $M$ is a Seifert fibre space over an orbifold $X$ and if $\hat{X}$ is an orbifold covering of $X$, there is a natural covering space $\bar{M}$ of $M$ with an orbit space $\hat{X}$ which should be thought of as the bundle over $\tilde{X}$ induced by the projection $\tilde{X} \rightarrow X$. Again this statement is clear if $M$ is a fibred solid torus or Klein bottle and follows easily for any Seifert fibre space. We are now in a position to prove the first significant fact about Seifert fibre spaces.

Lemma 3.1. Let $M$ be a Seifert fibre space without boundary. Then the universal covering $\tilde{M}$ of $M$ is homeomorphic to one of $S^{3}, \mathbb{R}^{3}$ or $S^{2} \times \mathbb{R}$. Further, the induced foliation of $\bar{M}$ by circles or lines gives $\hat{M}$ one of the following structures:

(i) a Seifert bundle over one of the orbifolds $S^{2}, S^{2}(p), S^{2}(p, q)$ where $p$ and $q$ are coprime;

(ii) a product line bundle over $\mathbb{R}^{2}$;

(iii) a product line bundle over $S^{2}$.

Proof. First suppose that the natural foliation of $\bar{M}$ is by circles, so that $\bar{M}$ is a Seifert fibre space. As $\hat{M}$ is simply connected, it has no proper coverings. Hence the base space of $\bar{M}$ is an orbifold $\tilde{X}$ with no proper coverings. The only such orbifolds are $S^{2}, \quad S^{2}(p), \quad S^{2}(p, q)$, where $p$ and $q$ are coprime, and $\mathbb{R}^{2}$. The case $\hat{X}=\mathbb{R}^{2}$ cannot occur, for then $\bar{M}$ would be $S^{1} \times \mathbb{R}^{2}$ and so not be simply connected. In the other cases, we write $\hat{X}=D_{1} \cup D_{2}$ where $D_{1}$ and $D_{2}$ are 2-discs with a possible interior cone point. Thus $\tilde{M}$ is the union of two fibred solid tori $T_{1}$ and $T_{2}$ where $T_{i}$ has base space $D_{i}$; and hence is a lens space. As $\tilde{M}$ is simply connected, it must be $S^{3}$.

If $\tilde{M}$ is foliated by lines, then $\tilde{M}$ is a line-bundle over its base space $\tilde{X}$ and $\tilde{X}$ is an orbifold without singularities. Again $\tilde{X}$ is also simply connected so that $\tilde{X}$ must be $S^{2}$ or $\mathbb{R}^{2}$. This gives cases (ii) or (iii) of the lemma.

The result of Lemma 3.1 has many consequences. A 3-manifold $M$ is called irreducible if any embedded 2-sphere in $M$ bounds a 3-ball, and is called 
$P^{2}$-irreducible if, in addition, $M$ contains no two-sided projective plane. Now $S^{3}$ and $\mathbb{R}^{3}$ are irreducible [1], and so any manifold covered by them must be $P^{2}$-irreducible [21]. It follows that a Seifert fibre space is $P^{2}$-irreducible unless it is covered by $S^{2} \times \mathbb{R}$. In particular, most Seifert fibre spaces cannot be a connected sum. In fact, we shall see later that the connected sum of two copies of $\mathbb{B} P^{3}$ is the only Seifert fibre space which is a connected sum.

Also every Seifert fibre space is aspherical (i.e. has all homotopy groups trivial except for $\pi_{1}$ ) unless it is covered by $S^{3}$ or $S^{2} \times \mathbb{R}$. This is because $\mathbb{R}^{3}$ is contractible. Note that these statements apply to all Seifert fibre spaces, not just those without boundary. For Lemma 3.1 can be applied to the interior of a Seifert fibre space with boundary.

Continuing with the same approach, we can now see the rather special structure of $\pi_{1}(M)$, when $M$ is a Seifert fibre space. For $\pi_{1}(M)$ acts on $\bar{M}$ preserving the natural foliation, so that there is an induced action of $\pi_{1}(M)$ on $\bar{X}$. This gives a natural homomorphism $\pi_{1}(M) \rightarrow \pi_{1}(X)$, where $\pi_{1}(X)$ denotes the orbifold fundamental group of $X$. The kernel $K$ of this map consists of covering translations of $\bar{M}$ which project to the identity map on $\bar{X}$. As $K$ acts freely on any one of the fibres of $\bar{M}$ we see that $K$ is infinite cyclic if $\bar{M}$ is not compact and is finite cyclic when $M$ is $S^{3}$. Thus we have shown the following.

Lemma 3.2. Let $M$ be a Seifert fibre space with base orbifold $X$. There is an exact sequence

$$
1 \longrightarrow K \longrightarrow \pi_{1}(M) \longrightarrow \pi_{1}(X) \longrightarrow 1
$$

where $K$ denotes the cyclic subgroup of $\pi_{1}(M)$ generated by a regular fibre. The group $K$ is infinite except in the cases where $M$ is covered by $S^{3}$.

Remarks. As $K$ is normal in $\pi_{1}(M)$, there are no base point problems with the statement that $K$ is generated by a regular fibre.

This exact sequence is another reason for regarding $M$ as a kind of bundle over $X$.

A boundary component $F$ of a 3-manifold $M$ is called incompressible if $F$ is not $S^{2}$ or $P^{2}$ and the natural map $\pi_{1}(F) \rightarrow \pi_{1}(M)$ is injective. This last condition is equivalent [21] to asserting that if $D$ is a 2-disc embedded in $M$ with $\partial D$ contained in $F$, then $\partial D$ must bound a 2-disc $D^{\prime}$ in $F$. An example of a 3-manifold with compressible boundary is the solid torus $S^{1} \times D^{2}$. A nice consequence of Lemma 3.2 is the following.

COROllary 3.3. The boundary of a Seifert fibre space $M$ is incompressible unless $M$ is homeomorphic to a solid torus or solid Klein bottle.

Proof. First, note that if $\partial M$ is non-empty, the interior of $M$ is non-compact and so cannot be covered by $S^{3}$. Nor can the interior of $M$ be covered by $S^{2} \times \mathbb{R}$ as neither $S^{2}$ nor $P^{2}$ can be boundary components of a Seifert fibre space. Hence a regular fibre in $M$ represents an element of infinite order in $\pi_{1}(M)$ and $M$ is $P^{2}$-irreducible. In particular, if $\partial M$ is compressible in $M$, it must have a compact boundary component $T$. For otherwise, each boundary component has an infinite cyclic fundamental group generated by a fibre and so is incompressible in $M$. Now $T$ must be a torus or Klein bottle. Hence surgery on $T$ using a 2-disc $D$ embedded in $M$ 
with $D \cap T=\partial D$, an essential loop in $T$, yields a 2-sphere or 2-sided projective plane $S$ in $M$. As $M$ is $P^{2}$-irreducible, $S$ must be a 2 -sphere and bound a 3-ball in $M$, and it follows easily that $M$ must be a solid torus or solid Klein bottle as required.

So far, we have not answered the natural question of which orbifolds can be the base of a Seifert fibre space. The answer is that any orbifold without corner reflectors can occur and, in general, it will be the base of many non-isomorphic Seifert bundles. If we start with a surface $X$ regarded as a 2-dimensional orbifold without singularities, then $X \times S^{1}$ is certainly a Seifert fibre space over $X$ and so is any other circle bundle over $X$. We will discuss shortly the question of classifying circle bundles over $X$.

Now we consider a general 2-dimensional orbifold $X$. Let $X^{\prime}$ denote $X$ minus the interior of a regular neighbourhood $N$ of the singular points. Let $\Sigma$ denote $X^{\prime} \cap N$, which is a union of intervals and circles. (Remember that $X$ need not be compact and may have boundary.) Any Seifert bundle over $X$ is the union of a Seifert bundle over $X^{\prime}$ with a Seifert bundle over $N$. Conversely, if we have a Seifert bundle over $X^{\prime}$ and a Seifert bundle over $N$, we can take their union and obtain a Seifert bundle over $X$, so long as there is an isomorphism between the two Seifert bundles when restricted to $\Sigma$. Note that a Seifert bundle over $X^{\prime}$ is simply a circle bundle over $X^{\prime}$ and there is always at least one such. Now we consider Seifert bundles over the components of $N$.

Let $D$ be a component of $N$ which is a disc neighbourhood of a cone point on $X$ and let $C$ denote $\partial D$. A Seifert bundle $T$ over $D$ exists whatever the cone angle at the cone point and $T$ will be a fibred solid torus. Hence it will restrict to a product circle bundle over $C$. Hence a circle bundle $\eta$ over $X^{\prime}$ extends to a Seifert bundle over $X^{\prime} \cup D$ if and only if $\eta$ is trivial when restricted to $C$.

The other possibility for a compact component of $N$ is an annulus $E$ where one of the boundary components of $E$ is a reflector circle. Call this component $C_{1}$ and the other boundary component $C_{2}$. See Fig. 3.4.

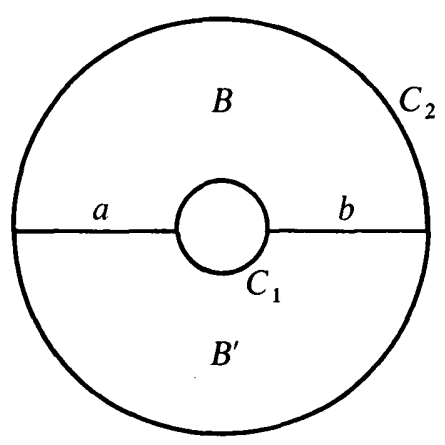

FIG. 3.4

We can divide $E$ into 2 -discs $B$ and $B^{\prime}$ by cutting $E$ along arcs $a$ and $b$ as shown in Fig. 3.4. Each disc, as an orbifold, has an arc of reflexion points in its boundary and so is clearly the quotient of the 2-disc $D^{2}$ by a reflexion. Hence, as was pointed out at the start of this section, there is a Seifert bundle over $B$ and over $B^{\prime}$, which is a solid Klein bottle in each case. The restriction of each Seifert bundle to each of the arcs $a$ and $b$ of $\partial B$ and $\partial B^{\prime}$ is a Moebius band. The critical fibre at the centre of the Moebius 
band projects to the point $a \cap C_{1}$ or $b \cap C_{1}$ as appropriate. Clearly, we can now take the union of the two Seifert bundles over $B$ and $B^{\prime}$ by identifying their restrictions to $a$ and $b$ and so obtain a Seifert bundle over $E$. There are two essentially distinct identifications possible. In one case the Seifert bundle over $E$ when restricted to $C_{1}$ or $C_{2}$ is a torus, and in the other case both restrictions give a Klein bottle. In either case, the total space of the Seifert bundle over $E$ is a twisted $I$-bundle over its restriction to $C_{1}$. (An $I$-bundle is a bundle with fibre the unit interval $I$. Such a bundle is called twisted if and only if it is non-trivial.) We conclude that every circle bundle over $X^{\prime}$ extends in a unique way to a Seifert bundle over $X^{\prime} \cup E$. This is also clearly the case for all the non-compact components of $N$ each of which must be a regular neighbourhood of a line of reflexion points.

We have now seen that any 2-dimensional orbifold without corner reflectors is the base of at least one Seifert fibre space. We have also seen that it is natural to construct a Seifert bundle over an orbifold $X$ by starting with a circle bundle over a surface and then glueing on pieces corresponding to the components of the singular set of $X$. I now want to discuss the invariants of a Seifert bundle and then the classification of Seifert bundles up to bundle isomorphism.

I will start by discussing the case when the base space is an orbifold without singularities. Thus one is simply considering circle bundles over a surface $X$. If $X$ is not a closed surface, then $X$ is homotopy equivalent to a wedge of circles, so that a bundle over $X$ is determined by its restriction to the corresponding loops in $X$. Now there are only two circle bundles over $S^{1}$ and their total spaces are the torus and the Klein bottle. Hence a circle bundle $\eta$ over $X$ determines a homomorphism $f: \pi_{1}(X) \rightarrow \mathbb{Z}_{2}$ such that if $\lambda: S^{1} \rightarrow X$ is a loop on $X$, then the restriction of $\eta$ to $S^{1}$ (strictly speaking the pull back $\lambda^{*} \eta$ of $\eta$ ) is the trivial circle bundle over $S^{1}$ if and only if $f([\lambda])$ is trivial. Clearly this gives a bijection between circle bundles over $X$ and homomorphisms $\pi_{1}(X) \rightarrow \mathbb{Z}_{2}$, which in turn correspond to elements of $H^{1}\left(X, \mathbb{Z}_{2}\right)$. This gives a precise classification of circle bundles over $X$, when two such are considered equivalent only if there is a bundle isomorphism between them which covers the identity map of $X$. If one regards two circle bundles over $X$ as equivalent simply if there is a bundle isomorphism between them, one has a much coarser equivalence relation with very few equivalence classes. But we need not concern ourselves with the details. See [46, 47 and 16].

Now we consider a circle bundle over a closed surface $X$. Such a bundle still determines a homomorphism $\phi: \pi_{1}(X) \rightarrow \mathbb{Z}_{2}$ and any such homomorphism can occur, but in order to determine the bundle $\eta$ one needs an extra invariant, denoted $b(\eta)$, which is the obstruction to the existence of a section of the bundle $\eta$. The invariant $b$ is an integer if the total space of $\eta$ is orientable and lies in $\mathbb{Z}_{2}$ otherwise. It can take any value and a circle bundle $\eta$ over $X$ is determined by the homomorphism $\phi: \pi_{1}(X) \rightarrow \mathbb{Z}_{2}$ and by $b(\eta)$. All these facts follow from standard obstruction theory. The obstruction $b(\eta)$ lies in $H^{2}(X ; \mathbb{Z})$, where the coefficients may be twisted. In fact $\pi_{1}(X)$ acts on $\mathbb{Z}$ via the homomorphism $\phi: \pi_{1}(X) \rightarrow \mathbb{Z}_{2}$, where we identify $\mathbb{Z}_{2}$ with Aut $(\mathbb{Z})$. This cohomology group is $\mathbb{Z}$ if $\phi$ is the orientation homomorphism of $X$ and is $\mathbb{Z}_{2}$ otherwise. Also note that $b(\eta)$ is a well-defined element of this cohomology group because the restriction of $\eta$ to the 1 -skeleton of $X$ will always admit a section. In the case when the total space of $\eta$ is orientable, one needs the orientation convention defined earlier to ensure that the sign of $b(\eta)$ is well defined. Unfortunately, this convention makes the obstruction element equal to $-b(\eta)$.

It is interesting to see how the invariant $b(\eta)$ shows up in the fundamental group 
of the total space $M$ of a circle bundle over a surface $X$. Assume that $X$ is not $S^{2}$ or $P^{2}$, so that $\pi_{2}(X)$ is zero and we have the exact sequence

$$
1 \longrightarrow K \longrightarrow \pi_{1}(M) \longrightarrow \pi_{1}(X) \longrightarrow 1
$$

where $K$ is infinite cyclic and is the fundamental group of the fibre. Let $k$ denote a generator of $K$. When $X$ is not closed, $\pi_{1}(X)$ is free, so that the exact sequence splits. If $X$ is closed, then $b(\eta)$ is the obstruction to this sequence splitting. For example, let $X$ be closed and orientable of genus $g$ and let $\bar{a}_{1}, \bar{b}_{1}, \ldots, \bar{a}_{g}, \bar{b}_{g}$ be a standard set of generators of $\pi_{1}(X)$, so that $\prod_{i=1}^{g}\left[\bar{a}_{i}, \bar{b}_{i}\right]=1$. Let $a_{i}$ be an element of $\pi_{1}(M)$ which projects to $\bar{a}_{i}$ and similarly for $b_{i}$. Then $\prod_{i=1}^{g}\left[a_{i}, b_{i}\right]=k^{r}$ for some $r$. If $M$ is oriented and $X$ and $S^{1}$ are oriented appropriately, one will have $r$ equal to $b(\eta)$. In all cases, $r$ is equal to $b(\eta)$ when calculated modulo 2 .

The only important fact that one needs to know about $b$ is the following naturality result, which explains how $b$ alters under finite coverings.

LEMMA 3.5. Let $\eta$ be a circle bundle over a closed surface $X$ with orientable total space $M$. Let $\bar{M}$ be a finite cover of $M$ of degree d, so that $\bar{M}$ is the total space of a circle bundle $\hat{\eta}$ over a surface $\hat{X}$. Let the covering $\hat{X} \rightarrow X$ have degree $l$ and let $m$ denote the degree with which fibres of $\dot{\eta}$ cover fibres of $\eta$ so that $\operatorname{lm}=d$.

Then $b(\tilde{\eta})=b(\eta) . l / m$.

This can be proved by studying either of the interpretations of $b$ already discussed. Note that $b(\tilde{\eta})$ must be an integer, so that the possible values of $l$ and $m$ are somewhat restricted.

If, in the above lemma, $M$ is non-orientable, then $b(\eta)$ is no longer an integer, so the above formula does not make sense. One can say what does happen, but all we will need to know is that if $\hat{M}$ is orientable, then $b(\tilde{\eta})$ is zero. Because of this, it is convenient to define a new integer invariant $e(\eta)$ of circle bundles over surfaces by letting $e(\eta)$ coincide with $-b(\eta)$ when $b(\eta)$ is an integer and setting $e(\eta)$ zero otherwise. For then the naturality result of Lemma 3.5 holds for $e(\eta)$ whether or not the total space of $\eta$ is orientable. Of course, we have lost some information by doing this, so we cannot forget about $b$. I will call $e(\eta)$ the Euler number of $\eta$. The minus sign in the definition of $e$ is to ensure that if $\eta$ is the unit tangent bundle of a closed orientable surface $F$, then $e(\eta)$ is equal to the Euler number $\chi(F)$ of $F$.

So far, we have only discussed circle bundles over a surface. Now we need to consider Seifert bundles over orbifolds. The first invariant of a Seifert bundle is the base orbifold $X$. Let $X^{\prime}$ denote $X$ with the interior of a regular neighbourhood $N$ of the singular set removed. Then we have a circle bundle over $X^{\prime}$, and we have already discussed the classification of such objects. (Note that if $X^{\prime}$ is a closed surface, then $X^{\prime}$ equals $X$.) There are no extra invariants to attach to the reflector lines and circles of $X$ because a circle bundle over $X^{\prime}$ extends uniquely to a Seifert bundle over the union of $X^{\prime}$ with the components of $N$ which contain reflector lines or circles. Our given Seifert bundle over $X$ is completed by adding the fibred solid tori corresponding to the cone points of $X$. Each fibred solid torus determines a pair of coprime integers $(p, q)$, the orbit invariants as described at the start of this section. One more invariant is needed to complete the classification of Seifert bundles in the 
case when the base space is a closed orbifold. This is a generalization of the invariant $b$ defined earlier for circle bundles over closed surfaces. As in that case, $b$ is an integer if the total space of the Seifert bundle is orientable and lies in $\mathbb{Z}_{2}$ otherwise. Seifert [59] showed that the invariants described here determine a Seifert bundle up to bundle isomorphism. Thus a Seifert bundle $\eta$ is specified by the base orbifold $X$, the circle bundle $\eta \mid X^{\prime}$, where $X^{\prime}$ is $X$ with the singular points removed, the orbit invariants of the critical fibres and the value of the invariant $b$. He did not discuss the case when $X$ has reflector curves, but one has the same result. Note that if $X$ has reflector curves, then $b$ should be defined to be zero. If $X$ has no reflector curves, then Seifert showed that $b$ could take any value except that if some pair of orbit invariants is $(2,1)$, then $b$ must be zero.

Unfortunately, the invariant $b$ no longer has any naturality properties under finite coverings. However, one can define another invariant called the Euler number $e(\eta)$ of a Seifert bundle $\eta$ in such a way that this invariant has a similar naturality property under finite coverings to that stated in Lemma 3.5. This has been done by Neumann and Raymond [44]. The Euler number is rational and is defined to be zero if the total space of $\eta$ is non-orientable. The precise result is the following.

THEOREM 3.6. Let $\eta$ be a Seifert bundle over a closed orbifold $X$ with total space $M$. Let $\bar{M}$ be a finite covering of $M$ of degree d, so that $\bar{M}$ is the total space of a Seifert bundle $\tilde{\eta}$ over an orbifold $\hat{X}$. Let the orbifold covering $\bar{X} \rightarrow X$ have degree $l$ and let $m$ be the degree with which regular fibres of $\dot{\eta}$ cover regular fibres of $\eta$, so that $I m=d$.

Then $e(\tilde{\eta})=e(\eta) .1 / m$.

The easy way to define $e$ is to proceed in the same way as in $\$ 2$, where we defined the Euler number $\chi(X)$ of an orbifold $X$. If a Seifert bundle $\eta$ is finitely covered by a circle bundle over a surface, we can define $e(\eta)$ by using the formula in Theorem 3.6. This will be independent of the choice of the covering $\dot{\eta}$ because $e$ satisfies the naturality property for coverings of bundles, Lemma 3.5. Recall that any good compact 2-dimensional orbifold is finitely covered by a surface. This means that any Seifert bundle with good compact base is finitely covered by a circle bundle over a surface. Thus we have defined $e(\eta)$ except when $\eta$ is a Seifert bundle over a bad orbifold, i.e. when $\eta$ is a Seifert bundle over the orbifolds $S^{2}(p)$ or $S^{2}(p, q)$, and $e$ clearly satisfies the naturality condition of Theorem 3.6. Now in the case of Seifert bundles with orientable total space, there is an equation connecting $e$ and $b$ (see three paragraphs ahead), and this equation can be used to define $e(\eta)$ when $\eta$ is a Seifert bundle over a bad orbifold.

A better approach to the Euler number $e$ of a Seifert bundle is to think of $e$ as the obstruction to a section. This means that one needs to discuss the idea of section of a Seifert bundle over an orbifold, but this is not difficult, see [66], and when it is done, one has an intrinsic definition of $e(\eta)$ for any Seifert bundle $\eta$. See also [44].

In $\$ 2$, when discussing the Euler number $\chi(X)$ of an orbifold $X$, I did not attempt to give an intrinsic definition of $\chi$ apart from the formula in terms of cone angles. However, there is a very simple idea which yields an intrinsic definition of $\chi$. In the case of a suface $X$, the Euler number $\chi(X)$ is the obstruction to the existence of a section of the unit tangent bundle of $X$. There is a natural idea of unit tangent bundle of an orbifold $X$ and it is a Seifert bundle over $X$. The Euler number $\chi(X)$ is simply the obstruction $e(\eta)$ to the existence of a section of the tangent bundle $\eta$ of $X$. 
Next I want to give the formula connecting the invariants $e$ and $b$. First we must define the Seifert invariants $(\alpha, \beta)$ of an isolated critical fibre of a Seifert fibre space whose orbit invariants are $(p, q)$. One defines $\alpha=p$ and $\beta$ is given by $\beta q \equiv 1$ $(\bmod p)$. As usual $\beta$ can be normalized so that $0<\beta<\alpha$, if desired. Clearly the orbit invariants determine the Seifert invariants and vice versa. The formula connecting $e(\eta)$ and $b(\eta)$ is

$$
e=-\left(b+\sum_{i=1}^{r} \beta_{i} / \alpha_{i}\right)
$$

where $\eta$ is a Seifert bundle over a closed orbifold $X$, the total space of $\eta$ is oriented and $\eta$ has $r$ critical fibres with normalised Seifert invariants $\left(\alpha_{i}, \beta_{i}\right)$. See [44]. Note that although this formula uses normalised Seifert invariants, one can use unnormalised invariants, so long as one redefines $b$. This idea is a significant part of the proof in [44] of Theorem 3.6.

In order to understand the significance of the Seifert invariant, recall from Lemma 3.2 that if $\eta$ is a Seifert bundle with base orbifold $X$ and total space $M$, there is an exact sequence

$$
1 \longrightarrow K \longrightarrow \pi_{1}(M) \longrightarrow \pi_{1}(X) \longrightarrow 1,
$$

where $K$ is the cyclic subgroup of $\pi_{1}(M)$ generated by a regular fibre. We consider the special case when $M$ is a fibred solid torus with orbit invariants $(p, q)$, so that the base orbifold $X$ is a cone. Let $k$ denote a generator of the infinite cyclic group $K$, let $\bar{x}$ denote a generator of $\pi_{1}(X)$, represented by $\partial X$, so that $\bar{x}^{p}=1$, and let $x$ denote an element of $\pi_{1}(M)$ which projects to $\bar{x}$. The significance of the Seifert invariant $\beta$ is that $x^{p}=k^{\beta}$ where $\beta q \equiv 1(\bmod p)$. This is best seen by considering the action of $\pi_{1}(M)$ on the universal covering space $\mathbb{R} \times D^{2}$ of $M$. If $u$ denotes a generator of $\pi_{1}(M)$, then, by construction of $T(p, q), u$ acts on $\mathbb{R} \times D^{2}$ by the product of a translation of $\mathbb{R}$ with a rotation through $2 \pi q / p$, and $u^{p}=k$. But $x$ acts on $\mathbb{R} \times D^{2}$ by the product of a translation of $\mathbb{R}$ with a rotation through $2 \pi / p$. As $u$ generates $\pi_{1}(M)$, $x$ must equal $u^{\beta}$ for some $\beta$, so the rotation angle of $x$ is $2 \pi q \beta / p$. Hence $\beta q \equiv 1$ $(\bmod p)$. Also $x^{p}=u^{\beta p}=k^{\beta}$ as claimed.

Now we can describe the fundamental group of a Seifert fibre space in some detail, using the invariants previously discussed. The presentations one obtains for $\pi_{1}(M)$ can be very forbidding, so I will simply illustrate what happens with a few examples. See [46] for complete details of the presentations when the base orbifold has no reflector curves.

Let $W$ be the surface obtained from the torus by removing the interior of a 2-disc. Thus $W$ has no singularities when regarded as an orbifold. The fundamental group of $W$ is free of rank two and we choose natural generators $\bar{u}$ and $\bar{v}$, so that $\partial W$ represents $[\bar{u}, \bar{v}]$ in $\pi_{1}(W)$. If $\eta$ is a circle bundle over $W$ with total space $E$, then the exact sequence

$$
1 \longrightarrow K \longrightarrow \pi_{1}(E) \longrightarrow \pi_{1}(W) \longrightarrow 1
$$

shows that $\pi_{1}(E)$ can be given the following presentation

$$
\left\{k, u, v: u^{-1} k u=k^{\delta}, v^{-1} k v=k^{\varepsilon}\right\},
$$

where $k$ is a generator of $K, u$ and $v$ project to $\bar{u}$ and $\bar{v}$ and $\delta$ and $\varepsilon$ are each 1 or -1 . 
The numbers $\delta$ and $\varepsilon$ determine whether or not $\eta$ is trivial when restricted to loops in $W$ representing $\bar{u}$ and $\bar{v}$.

Let $X$ be the orbifold whose underlying surface is the torus and which has one cone point with cone angle $2 \pi / p$. Thus $X=W \cup D$, where $D$ denotes the orbifold whose underlying surface is the 2-disc and which has one cone point. Let $\eta$ be a Seifert bundle over $X$ and denote the orbit and Seifert invariants respectively of its critical fibre by $(p, q)$ and $(\alpha, \beta)$. Let $M$ denote the total space of $\eta$, let $E$ denote the total space of $\eta \mid W$ and let $T(p, q)$ denote the total space of $\eta \mid D$. The previous paragraph gave a presentation for $\pi_{1}(E)$ and we know that $\pi_{1}(T(p, q))$ is infinite cyclic. Further $\pi_{1}(T(p, q))$ has natural generators $k$ and $x$ where $k$ is represented by any non-critical fibre of $T(p, q)$ and $x$ projects to the element $\bar{x}$ of $\pi_{1}(D)$ represented by $\partial D$. Note that $x$ is uniquely determined by the equation $x^{x} k^{\beta}=1$. Putting these facts together yields the following presentation for $\pi_{1}(M)$ :

$$
\left\{k, u, v, x: u^{-1} k u=k^{\delta}, v^{-1} k v=k^{k}, x^{-1} k x=k, x^{x} k^{\beta}=1,[u, v] x=k^{b}\right\},
$$

where $b$ denotes the $b$-invariant of $\eta$. Note that we have the relation $x^{x} k^{\beta}=1$ rather than $x^{\alpha}=k^{\beta}$, in order to make our presentation coincide with the conventional one.

For another example, let $X_{1}$ be the orbifold with underlying surface homeomorphic to $W$, with no cone points and with $\partial W$ as a reflector circle. We can regard $X_{1}$ as $W \cup A$ where $A$ is the orbifold with underlying surface the annulus and one reflector circle, as in Fig. 2.1. Recall that $\pi_{1}(A)$ is isomorphic to $\mathbb{Z} \times \mathbb{Z}_{2}$, and $\pi_{1}\left(X_{1}\right)$ is isomorphic to $\pi_{1}(W){ }_{2} \pi_{1}(A)$.

Let $\eta$ be a Seifert bundle over $X_{1}$ with total space $M$, let $E$ denote the total space of $\eta \mid W$ and let $H$ denote the total space of $\eta \mid A$. As the restriction of $\eta$ to $\partial W$ must be trivial, for any circle bundle over $W$, we see that $\pi_{1}(M)$ is isomorphic to $\pi_{1}(E){ }_{\mathbf{z} \times \mathbf{z}} \pi_{1}(H)$. Our discussion of Seifert bundles over $A$ earlier in this section shows that $\pi_{1}(H)$ is also free abelian of rank two and contains the amalgamating $\mathbb{Z} \times \mathbb{Z}$ as a subgroup of index two. One can, of course, give a presentation for $\pi_{1}(M)$ in terms of generators and relations but the above expression for $\pi_{1}(M)$ seems more illuminating.

Now let $\eta$ be a Seifert bundle with compact total space $M$, whose base orbifold $X$ has a geometric structure and so is finitely covered by an orbifold without singularities by the discussion at the end of $\$ 2$. Thus $M$ is finitely covered by a circle bundle over a surface. By taking at most two further double covers, we can obtain a circle bundle $\bar{M}$ over a surface $\hat{X}$ where $\bar{M}$ and $\bar{X}$ are both orientable. Now such a bundle is trivial if and only if its Euler number is zero. Thus we see that there is a finite cover $\bar{M}$ of $M$ which is a trivial circle bundle (with the induced Seifert bundle structure) if and only if $e(\eta)=0$.

The only orbifolds without corner reflectors which do not possess a geometric structure are of the form $S^{2}(p)$ or $S^{2}(p, q)$, where $p$ and $q$ are distinct. The Euler number of a Seifert bundle over $S^{2}(p)$ is of the form $b+\beta / p$ where $1 \leqslant \beta<p$ and $b$ is an integer, and so cannot be zero. Also, the Euler number of a Seifert bundle over $S^{2}(p, q)$ is of the form $b+\beta_{1} / p+\beta_{2} / q$ where $b$ is an integer and $1 \leqslant \beta_{1}<p$, $1 \leqslant \beta_{2}<q$ and this also cannot be zero. (Recall that $\beta_{1}$ and $p$ are coprime as are $\beta_{2}$ and $q$ ). Putting this together with the result of the previous paragraph, we obtain the following.

LEMMA 3.7. If a Seifert bundle $\eta$ has compact total space $M$, then $M$ possesses a finite covering which is a trivial circle bundle over a surface, with the induced Seifert bundle structure, if and only if $e(\eta)=0$. 
I want to end this section by discussing briefly the homeomorphism classification of Seifert fibre spaces. We saw earlier that $S^{3}$ can be given Seifert bundle structures with base orbifolds $S^{2}, S^{2}(p)$ or $S^{2}(p, q)$, for any $p$ and $q$ which are coprime, so that infinitely many distinct Seifert bundles have total space $S^{3}$. However, it is also true that for a large class of Seifert fibre spaces, they are homeomorphic if and only if they have isomorphic Seifert bundle structures as the following result shows.

THEOREM 3.8. Let $M$ be a compact 3-manifold homeomorphic to two nonisomorphic Seifert bundles. Then one of the following cases occur:

(a) $M$ is covered by $S^{3}$ or $S^{2} \times \mathbb{B}$;

(b) $M$ is covered by $S^{1} \times S^{1} \times S^{1}$;

(c) $M$ is $S^{1} \times D^{2}$ or a I-bundle over the torus or Klein bottle.

REMARK. In cases (a), (b) and (c), only some of the manifolds defined admit nonunique Seifert bundle structures. I will say more about this after the proof of the theorem.

Proof. We start by assuming that $M$ is not covered by $S^{3}$ or $S^{2} \times \mathbb{R}$. Hence all the Seifert bundles under consideration have a base orbifold with a geometric structure modelled on $E^{2}$ or $H^{2}$ and we have the usual exact sequence

$$
1 \longrightarrow K_{i} \longrightarrow \pi_{1}\left(N_{i}\right) \longrightarrow \pi_{1}\left(X_{i}\right) \longrightarrow 1,
$$

for any Seifert bundle $N_{i}$ homeomorphic to $M$, where $K_{i}$ is infinite cyclic.

Let $N_{1}$ and $N_{2}$ be Seifert bundles homeomorphic to $M$. Thus there is a homeomorphism $N_{1} \rightarrow N_{2}$ and hence an isomorphism $\phi: \pi_{1}\left(N_{1}\right) \rightarrow \pi_{1}\left(N_{2}\right)$. If $\phi\left(K_{1}\right)=K_{2}$, then by taking quotients, $\phi$ induces an isomorphism $\psi: \pi_{1}\left(X_{1}\right) \rightarrow \pi_{1}\left(X_{2}\right)$. Because $\phi$ was induced by a homeomorphism, it follows that $\psi$ sends peripheral elements of $\pi_{1}\left(X_{1}\right)$ to peripheral elements of $\pi_{1}\left(X_{2}\right)$. Hence it follows that $\psi$ is induced by an orbifold isomorphism $X_{1} \rightarrow X_{2}$, [76 and 34]. Now it can be shown that $\phi$ is induced by an isomorphism of Seifert bundles. Hence if $N_{1}$ and $N_{2}$ are distinct Seifert bundles, we cannot have $\phi\left(K_{1}\right)=K_{2}$. Hence, by interchanging $N_{1}$ and $N_{2}$ if necessary, we can suppose that $\phi\left(K_{1}\right)$ is not contained in $K_{2}$. Now the image of $\phi\left(K_{1}\right)$ in $\pi_{1}\left(X_{2}\right)$ must be a non-trivial cyclic normal subgroup of $\pi_{1}\left(X_{2}\right)$.

If $\pi_{1}\left(X_{2}\right)$ is a discrete group of isometries of $H^{2}$ with a non-trivial cyclic normal subgroup $L$, one needs to consider five cases depending on whether a generator of $L$ is a rotation, hyperbolic translation, parabolic isometry, reflexion or glide-reflexion. In all cases, one finds that $\pi_{1}\left(X_{2}\right)$ is either finite or is a finite extension of $\mathbb{Z}$, by using similar methods to those used to prove Lemma 1.10. Hence $X_{2}$ has a finite orbifold covering $\hat{X}_{2}$ with $\pi_{1}\left(\hat{X}_{2}\right)$ equal to 1 or $\mathbb{Z}$. Hence $M$ has a finite covering with fundamental group $\mathbb{Z}, \quad \mathbb{Z} \times \mathbb{Z}$ or the Klein bottle group. It follows that we are in case (c) of the theorem. See [21]. Note that the non-trivial $D^{2}$-bundle over $S^{1}$ has been omitted from case (c) as this can only be Seifert fibred in one way as a fibred solid Klein bottle.

If $\pi_{1}\left(X_{2}\right)$ is a discrete group of isometries of $E^{2}$, it may very well have a cyclic normal subgroup. We recall that every discrete isometry group of $E^{2}$ is a finite 
extension of its translation subgroup which must be $1, \mathbb{Z}$ or $\mathbb{Z} \times \mathbb{Z}$. In the first two cases, we deduce that case (c) of the theorem holds. In the third case, one can show easily that a non-trivial cyclic normal subgroup $L$ of $\pi_{1}\left(X_{2}\right)$ must be infinite. By taking two double covers of $X_{2}$, if necessary, we can suppose that $K_{2}$ is central in $\pi_{1}\left(N_{2}\right)$ and that $L$ is central in $\pi_{1}\left(X_{2}\right)$. Let $\bar{L}$ denote the pre-image in $\pi_{1}\left(N_{2}\right)$ of $L$ in $\pi_{1}\left(X_{2}\right)$. Then $\bar{L}$ is isomorphic to $\mathbb{Z} \times \mathbb{Z}$ and is central in $\pi_{1}\left(X_{2}\right)$. Further the quotient $\pi_{1}\left(N_{2}\right) / \bar{L}$ is isomorphic to $\pi_{1}\left(X_{2}\right) / L$ and so is a finite extension of $\mathbb{Z}$. By taking a further finite cover of $X_{2}$, if necessary, we can arrange that $\pi_{1}\left(N_{2}\right) / \bar{L}$ is cyclic. Now it follows that $\pi_{1}\left(N_{2}\right)$ is isomorphic to $\mathbb{Z} \times \mathbb{Z} \times \mathbb{Z}$, so that $N_{2}$ is $S^{1} \times S^{1} \times S^{1}$. This completes the proof of Theorem 3.8.

The conclusion to be drawn from Theorem 3.8 is that in most cases, if two Seifert bundles are homeomorphic then they are isomorphic. In fact, the arguments used show that a still stronger result holds - namely that for most manifolds which admit a Seifert fibration, this fibration is unique up to homotopy. The precise statement is as follows, and was first proved in [48] and [72].

TheOREM 3.9. Let $M$ be a compact Seifert fibre space and let $f: M \rightarrow N$ be a homeomorphism. Then $f$ is homotopic to a fibre preserving homeomorphism (and hence un isomorphism of Seifert bundles) unless one of the following occurs.

(a) $M$ is covered by $S^{3}$ or $S^{2} \times \mathbb{R}$,

(b) $M$ is covered by $S^{1} \times S^{1} \times S^{1}$,

(c) $M$ is $S^{1} \times D^{2}$ or a I-bundle over the torus or Klein bottle.

REMARK. As with the previous theorem not all the manifolds defined in (a), (b) and (c) are exceptional. Note that $S^{1} \times S^{1} \times S^{1}$ plainly is one of the exceptions to Theorem 3.9, but only one Seifert bundle can occur, namely the trivial $S^{1}$-bundle over $S^{1} \times S^{1}$. Thus $S^{1} \times S^{1} \times S^{1}$ is not an exception to Theorem 3.8. The same comments apply to $S^{1} \times S^{1} \times I$. However, the Klein bottle can be thought of in two quite different ways as a Seifert fibre space. It can be regarded as the non-trivial circle bundle over $S^{1}$ or as a Seifert fibre space with base orbifold an interval, and two critical fibres corresponding to the end points. Hence an $I$-bundle over the Klein bottle may have two Seifert bundle structures. (Note that the Moebius band is naturally a Seifert fibre space over an interval with one critical fibre, and that the Klein bottle is the union of two Moebius bands.)

Proof of Theorem 3.9. We will assume that cases (a), (b) and (c) do not occur. Recall the exact sequence

$$
1 \longrightarrow K \longrightarrow \pi_{1}(M) \longrightarrow \pi_{1}(X) \longrightarrow 1,
$$

where $X$ is the base orbifold of $M$ and $K$ is the infinite cyclic subgroup of $\pi_{1}(M)$ generated by a regular fibre. The homeomorphism $f$ induces an isomorphism $f_{*}$ of $\pi_{1}(M)$ (defined up to conjugacy). As we have excluded cases (a), (b) and (c), Theorem 3.8 shows that $f_{*}(K)=K$. Hence $f_{*}$ induces an isomorphism $\psi$ of $\pi_{1}(X)$ with itself which sends peripheral elements to peripheral elements. Hence $\psi$ is induced by an orbifold homeomorphism $h$ of $X$, (see [76 and 34]). As $\psi$ comes from 
an isomorphism $f_{*}$ of $\pi_{1}(M)$, one can define a homeomorphism $\bar{h}$ of $M$ which preserves fibres and projects to $h$, such that $\bar{h}_{*}=f_{*}$. As $M$ is aspherical, $f$ is homotopic to $\bar{h}$, proving Theorem 3.9.

A further refinement of Theorem 3.9 can be made so long as $M$ is Haken. For then homotopic homeomorphisms are isotopic. See $[74$ or 30]. Hence a homeomorphism of Haken Seifert fibre spaces is isotopic to a fibre preserving map unless one is in one of the exceptional cases of Theorem 3.9.

\section{\$4. The eight 3-dimensional geometries}

The purpose of this section is to describe the eight 3-dimensional geometries and their basic properties. These are also discussed by Thurston [66] and some of them are discussed by Auslander, Green and Hahn [2]. In $\$ 5$, I will discuss more carefully what is being classified and why these eight geometries are the only ones in which we need be interested. For each of the geometries, except for hyperbolic geometry, I will describe the structure of manifolds possessing the appropriate geometric structure. It turns out that for six of the geometries, all the closed manifolds are Seifert fibre spaces. It is also true that every Seifert fibre space admits a geometric structure and this will be proved in $\S 5$.

We will also prove in $\$ 5$ that if a closed 3-manifold admits a geometric structure then the geometry involved is unique. This gives a very nice division of closed Seifert fibre spaces into six classes according to which geometric structure they admit. In the 2-dimensional case, the appropriate geometry for a given closed surface $F$ was determined by the Euler number $\chi(F)$ and whether this is positive, zero or negative. When we turn to Seifert bundles, we find that the appropriate geometry can be determined from the two invariants $\chi$ and $e$, where $\chi$ is the Euler number of the base orbifold and $e$ is the Euler number of the Seifert bundle, according to the following table. The determining factors are whether $\chi$ is positive, zero or negative and whether $e$ is zero or not.

TABLE 4.1

THE APPROPRIATE GEOMETRIES FOR SEIFERT BUNDLLES WITH CLOSED TOTAL SPACE

\begin{tabular}{llll}
\hline & $\underline{\chi>0}$ & $\underline{\chi=0}$ & $\underline{\chi<0}$ \\
$e=0$ & $S^{2} \times \mathbb{R}$ & $E^{3}$ & $H^{2} \times \mathbb{R}$ \\
$e \neq 0$ & $S^{3}$ & $\mathrm{Nil}$ & $\widetilde{\mathrm{SL}_{2} \mathbb{R}}$ \\
\hline
\end{tabular}

Note that some closed manifolds admit many Seifert bundle structures, but for a given closed manifold, all its Seifert bundle structures will belong to the same geometry.

There is another structure which we will come upon in this section which is very similar to a Seifert fibre structure. Recall that a Seifert fibration of a manifold $M$ is an expression of $M$ as a circle "bundle" over a 2-dimensional orbifold. One can equally well consider 3-manifolds which are "bundles" over a 1-dimensional orbifold with 2-dimensional fibre.

A 1-dimensional orbifold can only have one kind of singular point which I will call a reflector point. The quotient of a circle by a reflexion is an orbifold with underlying space the closed unit interval and with both endpoints as reflector points. 
Clearly, there are only four compact 1-dimensional orbifolds, namely $S^{1}$ which has no singular points, and the unit interval with zero, one or two reflector points.

If $F$ is a surface and $p: F \times I \rightarrow I$ is the natural projection, then $F \times I$ is, in the usual sense, a bundle over $I$ with fibre $F$. If $F \overline{\times} I$ is a twisted $I$-bundle over $F$, there is a double cover $L$ of $F$ and a projection $L \times I \rightarrow F \tilde{x} I$ which is also a double covering. The covering involution on $L \times I$ can be chosen to be the product of a free involution of $L$ with a reflexion of $I$. Now $F \tilde{x} I$ has a natural foliation by the surfaces which are the images of the surfaces $L \times\{t\}$. All these surfaces in $F \bar{x} I$ are homeomorphic to $L$ except for one which is a copy of $F$ and is one-sided in $F \dot{x} I$. The space obtained from $F \bar{x} I$ by identifying each of these surfaces to a point is a closed interval and naturally has the structure of an orbifold with one reflector point corresponding to the one-sided leaf. We will say that $F \tilde{x} I$ is a surface bundle over this 1-dimensional orbifold.

In general, we can define a surface bundle over a 1-dimensional orbifold $X$ to be a 3-manifold $M$ with a foliation by surfaces such that each leaf has a neighbourhood of one of the above two types. A leaf is critical if its neighbourhood is twisted and regular otherwise. If the base orbifold $X$ is a circle, then $M$ is clearly a bundle, in the usual sense, over $X$. If $X$ is an interval with no reflector points, then $M$ is simply a product $F \times X$, where $F$ denotes any leaf of $M$. If $X$ is an interval with one reflector point, then $M$ has the structure of a twisted $I$-bundle over the critical leaf. Finally, if $X$ is an interval with two reflector points, then we can decompose $X$ as $X_{1} \cup X_{2}$ where each $X_{i}$ is an interval with only one reflector point. Hence $M$ is naturally the union of two twisted $I$-bundles where each $I$-bundle is the total space of the restriction of the given bundle to $X_{1}$ or $X_{2}$.

If $M^{3}$ has a foliation by surfaces all of which are compact, it follows that each leaf has a neighbourhood of one of the above two types, so $M$ automatically has the structure of a surface bundle over a 1-dimensional orbifold. Of course, a foliation of $M$ by non-compact surfaces need not be of this special type. However a useful fact to note is that if the induced foliation on a finite cover $\bar{M}$ of $\bar{M}$ gives $\bar{M}$ the structure of a surface bundle over a 1-dimensional orbifold, then $M$ itself must have this structure.

I want to end this discussion by saying a little more about bundles over the circle. If $F$ is any space and $h: F \rightarrow F$ is a homeomorphism, one can construct a bundle $M$ over $S^{1}$ with fibre $F$ by starting with $F \times I$ and glueing $F \times\{1\}$ to $F \times\{0\}$ by the identification $(x, 1)=(h(x), 0)$. The homeomorphism $h$ is called a glueing map for the bundle.

If $F$ is a surface and $h$ is periodic, then $M$ is naturally a Seifert fibre space, where a typical fibre consists of the circle $\bigcup_{n \in Z} h^{n}(\{x\} \times I)$ for fixed $x$ in $F$. The base orbifold of this Seifert bundle is the quotient of $F$ by the action of $h$. This is one way in which a 3-manifold can simultaneously have the structures of a Seifert fibre space and of a bundle over a 1-dimensional orbifold.

Given any bundle over $S^{1}$ with fibre $F$, glueing map $h$ and total space $M$, one can construct a finite cover of $M$ by taking $d$ copies of $F$ and glueing the $i$-th copy of $F \times\{1\}$ to the $(i+1)$-th copy of $F \times\{0\}$ using $h$, where $i$ is to be interpreted modulo $d$. Clearly this new object is a $d$-fold covering of $M$ and is also a bundle over $S^{1}$ with fibre $F$ and glueing map $h^{d}$. It follows that if $h$ is periodic, then $M$ is finitely covered by $F \times S^{1}$, so that the Seifert bundle structure $\eta$ on $M$ has $e(\eta)=0$.

It is easy to show that if $h$ and $h^{\prime}$ are isotopic homeomorphisms of the surface $F$, then the bundles with glueing maps $h$ and $h^{\prime}$ are isomorphic. It is also known that 
homotopic homeomorphisms of a closed surface $F$ are isotopic [10]. Hence, for a closed surface $F$, the action of $h$ on $\pi_{1}(F)$ suffices to determine the isomorphism class of the bundle with glueing map $h$. If $h$ is homotopically periodic i.e. the $n$-th iterate $h^{n}$ of $h$ is homotopic to the identity map of $F$, then $h$ is homotopic to a periodic homeomorphism $h^{\prime}$ of $F$. This is easy if $F$ is $S^{2}$ or $P^{2}$, and follows from Theorem 2.6 in the remaining cases. It follows that if $M$ is the total space of a bundle over $S^{1}$ with fibre a closed surface $F$ and with homotopically periodic glueing map, then $M$ is a Seifert fibre space.

The geometry of $E^{3} . \quad E^{3}$ itself is a familiar object, but the special properties of groups of isometries of $E^{3}$ are less well known. With respect to the standard coordinates in $E^{3}$, any isometry $\alpha$ of $E^{3}$ can be expressed as $\alpha(\mathbf{x})=A \mathbf{x}+\mathbf{b}$, where $A$ is an orthogonal $3 \times 3$ real matrix and $\mathbf{b}$ is a vector in $E^{3}$. As in the 2-dimensional case, the map $\alpha \mapsto A$ defines a surjective homomorphism $\operatorname{Isom}\left(E^{3}\right) \rightarrow \mathrm{O}(3)$ with kernel equal to the group of translations of $E^{3}$. This gives the exact sequence

$$
0 \longrightarrow \mathbb{R}^{3} \longrightarrow \operatorname{Isom}\left(E^{3}\right) \longrightarrow \mathrm{O}(3) \longrightarrow 1 \text {. }
$$

The geometric description of isometries of $E^{3}$ differs from that in dimension two. For example, an orientation preserving isometry of $E^{3}$ is a screw motion consisting of the composite of a translation with a rotation about a line left invariant by the translation. Thus both translations and rotations are special cases of this type of isometry.

Recall that if $G$ is a discrete group of isometries of $E^{2}$, then its translation subgroup is of finite index. In higher dimensions, the analogous result is false. For example, if $G$ is the discrete infinite cyclic group of isometries of $E^{3}$ generated by a screw motion whose rotation angle is incommensurable with $\pi$, then the translation subgroup of $G$ is trivial. However, the famous Bieberbach theorems $[3,75]$ do tell us that if $G$ is a discrete group of isometries of $E^{n}$, then $G$ has a free abelian subgroup of finite index in $G$, and of rank not exceeding $n$. Using this result, it is not hard to show that if $G$ is a discrete group of isometries of $E^{3}$, then either the translation subgroup of $G$ is of finite index in $G$ or $G$ is a finite extension of $\mathbb{Z}$. One needs to show that if two screw motions commute, and if neither is a translation or rotation, then their axes coincide.

We can now discuss 3-manifolds with a complete Euclidean (or flat) structure i.e. a geometric structure modelled on $E^{3}$. Thus we are interested in a discrete group $G$ of isometries of $E^{3}$ which acts freely. In particular, $G$ is torsion free.

If $G$ is a finite extension of $\mathbb{Z}$, it follows that $G$ itself is infinite cyclic and so $E^{3} / G$ is the interior of a solid torus or solid Klein bottle. Thus $E^{3} / G$ can be given the structure of a Seifert fibre space. However, this structure need not be related to the group action. If $G$ is generated by a screw motion with axis $l$ and rotation angle commensurable with $\pi$, then $E^{3} / G$ is Seifert fibred by circles which are the projections of all the lines in $E^{3}$ parallel to $l$. But if $G$ is generated by a screw motion with rotation angle incommensurable with $\pi$, then the foliation of $E^{3}$ by lines parallel to $l$ descends to a foliation of $E^{3} / G$ by lines and not circles. Note that if $G$ is infinite cyclic and acts orientation reversingly on $E^{3}$, then there is always a natural Seifert fibration on $E^{3} / G$.

If $G$ is not a finite extension of $\mathbb{Z}$, then its translation subgroup $T$ has finite index by our earlier remarks. If $T$ is isomorphic to $\mathbb{Z} \times \mathbb{Z}$, it follows that $G$ itself is 
isomorphic to $\mathbb{Z} \times \mathbb{Z}$ or the Klein bottle group, and that $E^{3} / G$ is a line bundle over the torus or Klein bottle. These spaces can be Seifert fibred and there are natural foliations of $E^{3} / G$ consisting of circles coming from a $G$-invariant family of parallel straight lines in $E^{3}$.

Finally, if $T$ is isomorphic to $\mathbb{Z} \times \mathbb{Z} \times \mathbb{Z}$ and $G$ acts freely on $E^{3}$ it is a somewhat surprising fact that there is still a direction in $E^{3}$ left invariant by $G$ and that $E^{3} / G$ therefore admits a natural Seifert fibration. The only argument I know for proving this uses the classification of finite subgroups of SO (3) (see [75]), and is not as conceptual as I would like. However, it is not difficult so I will give the outline.

Let $G$ be a discrete group of isometries of $E^{3}$ with translation subgroup $T$ isomorphic to $\mathbb{Z} \times \mathbb{Z} \times \mathbb{Z}$. Thus one has the exact sequence

$$
1 \longrightarrow T \cong \mathbb{Z} \times \mathbb{Z} \times \mathbb{Z} \longrightarrow G \longrightarrow H \longrightarrow 1,
$$

where $H$ is a finite subgroup of $\mathrm{O}(3)$. The action of $G$ on $T$ by conjugation induces an action of $H$ on $T$ and we will show that some cyclic subgroup $C$ of $T$ is left invariant by this action. Now $C$ is a group of translations and so determines a direction in $E^{3}$ which must be left invariant by $G$. Further, as $G$ contains translations in this direction, namely the elements of $C$, it follows that the parallel lines in this direction descend to give a Seifert fibration on $E^{3} / G$, as required. It remains to prove the existence of the cyclic subgroup $C$ of $T$. Note that such a subgroup need not exist in general. It is crucial that $G$ is torsion free.

First suppose that $H$ is an orientation preserving subgroup of $\mathrm{O}(3)$, that is, a subgroup of SO (3). The classification of subgroups of SO (3) [75] tells us that $H$ is cyclic or dihedral or is the orientation preserving symmetry group of one of the five regular solids. As pointed out in $\$ 2$, there are only three distinct such symmetry groups and these are naturally isomorphic to the spherical triangle groups, $\Delta(2,3,3), \Delta(2,3,4)$ and $\Delta(2,3,5)$. We also know that the action of $H$ on $T$ gives an isomorphism of $H$ with a subgroup of $\mathrm{SL}_{3}(\mathbb{Z})$ and, from now on, we will identify $H$ with this subgroup. If $H$ is cyclic of order $n$ with generator $h$, then $h$ will have an eigenvalue equal to 1 or -1 and a corresponding integral eigenvector. This gives the required subgroup $C$ of $T$. Further if $n$ is not equal to 2, the subgroup $C$ is unique and if $n=2$, then $h$ has one eigenvalue equal to 1 , so that the corresponding subgroup $C_{1}$ of $T$ is again unique. It follows from this that if $H$ is dihedral, then the unique subgroup $C$ or $C_{1}$ of $T$ left invariant by the cyclic subgroup of $H$ of index two must also be left invariant by $H$, as required.

If $H$ is neither cyclic nor dihedral, it is clear that there is no cyclic subgroup of $T$ left invariant by $H$. So we need to eliminate the possibility that $H$ can be $\triangle(2,3,3)$, $\Delta(2,3,4)$ or $\Delta(2,3,5)$ using the fact that $G$ is torsion free, which has not appeared so far in our proof. As $\Delta(2,3,3)$ is isomorphic to a subgroup of $\Delta(2,3,4)$ and of $\Delta(2,3,5)$ it will suffice to eliminate the group $\Delta(2,3,3)$, which is also isomorphic to the alternating group $A_{4}$. The following result does this for us.

LEMMA 4.2. Let $G$ be a discrete group of orientation preserving isometries of $E^{3}$ with translation subgroup $T$ isomorphic to $\mathbb{Z} \times \mathbb{Z} \times \mathbb{Z}$ and with $G / T$ isomorphic to $A_{4}$. Then $G$ contains an element of order three.

Proof. Let $\alpha, \beta$ and $\gamma$ be those elements in $A_{4}$ of order two and let $\delta$ be an element of $A_{4}$ of order three such that $\delta \alpha \delta^{-1}=\beta, \delta \beta \delta^{-1}=\gamma, \delta \gamma \delta^{-1}=\alpha$. Now $A_{4}$ acts on $T$ and for this action $\alpha$ must have exactly one eigenvalue equal to 1 . Let $x$ 
be a corresponding integral eigenvector. Similarly, let $y$ and $z$ be eigenvectors with eigenvalue 1 for $\beta$ and $\gamma$ respectively. By taking $x, y$ and $z$ not to be multiples of any other vector and by, if necessary, multiplying some of these vectors by -1 we can ensure that the action of $\delta$ sends $x$ to $y, y$ to $z$, and $z$ to $x$. Hence $x+y+z$ is clearly fixed by $\delta$. Now we need to change to multiplicative notation. Let $d$ denote an element of $G$ which projects to $\delta$ in $A_{4}$. Then we know that $d x d^{-1}=y$, $d y d^{-1}=z, d z d^{-1}=x$ and $d^{3}$ lies in $T$. Now $d^{3}$ commutes with $d$ so that $d^{3}$ must be some power of $x y z$. If $d^{3}=1$, the lemma is proved. Otherwise $d^{3}=(x y z)^{k}$ for some non-zero integer $k$. Now I claim that the element $x^{-k} d$ of $G$ has order three. It is certainly non-trivial as it projects to $\delta$ in $A_{4}$ and we have

$$
\left(x^{-k} d\right)^{3}=x^{-k} d x^{-k} d x^{-k} d=x^{-k}\left(d x^{-k} d^{-1}\right)\left(d^{2} x^{-k} d^{-2}\right) d^{3}=x^{-k} y^{-k} z^{-k} d^{3}=1 \text {. }
$$

We have seen that if $G$ is a discrete group of isometries of $E^{3}$ with translation subgroup $T$ isomorphic to $\mathbb{Z} \times \mathbb{Z} \times \mathbb{Z}$ and if $G$ acts orientation preservingly on $E^{3}$, then $E^{3} / G$ is naturally a Seifert fibre space. We can now complete the proof that the same result holds when $G$ acts orientation reversingly. Recall the exact sequence

$$
1 \longrightarrow T \cong \mathbb{Z} \times \mathbb{Z} \times \mathbb{Z} \longrightarrow G \longrightarrow H \longrightarrow 1,
$$

where $H$ is a finite subgroup of $\mathrm{O}(3)$ and let $H_{1}$ be the orientation preserving subgroup of $H$. The previous discussion shows that there is a cyclic subgroup $C$ of $T$ left invariant by $H_{1}$. Further, $C$ is unique unless $H_{1}$ is cyclic of order two or dihedral of order 4 , that is, isomorphic to $\mathbb{Z}_{2} \times \mathbb{Z}_{2}$. If $H_{1}$ is isomorphic to $\mathbb{Z}_{2}$ there is a unique cyclic subgroup $C$ of $T$ left pointwise fixed by $H_{1}$. Hence $C$ must also be left invariant by $H$. If $H_{1}$ is isomorphic to $\mathbb{Z}_{2} \times \mathbb{Z}_{2}$, one can show that there are exactly three cyclic subgroups $C_{1}, C_{2}$ and $C_{3}$ of $T$ which are left invariant by $H_{1}$. Now $H / H_{1}$ must permute these and as $H / H_{1}$ has order two, at least one of $C_{1}, C_{2}$ and $C_{3}$ is left invariant. This is the cyclic subgroup of $T$ left invariant by $H$, which we are looking for. We have proved:

THEOREM 4.3. Let $G$ be a non-cyclic discrete group of isometries of $E^{3}$ which acts freely. Then $G$ leaves invariant some family of parallel straight lines in $E^{3}$ and $E^{3} / G$ is Seifert fibred by circles which are the images of these lines.

We can give more detail about the structure of $M=E^{3} / G$. Theorem 4.3 implies that $E^{3}$ has a product structure $E^{3}=E^{2} \times E^{1}$ preserved by $G$. Consider the natural projection $E^{3} \rightarrow E^{2}$. Then the action of $G$ on $E^{3}$ descends to an orthogonal action on $E^{2}$ whose quotient space is the base orbifold $X$ of $M$. If $M$ is compact, it is finitely covered by $E^{3} / T$, where $T$ is the translation subgroup of $G$, so that the natural Seifert bundle structure $\eta$ on $M$ is finitely covered by a trivial circle bundle over the torus $S^{1} \times S^{1}$. The naturality properties of the Euler numbers imply that both $e(\eta)$ and $\chi(X)$ are zero.

We have just used the $G$-invariant product structure $E^{3}=E^{2} \times E^{1}$ to deduce that $M$ has a Seifert fibre space structure. It is equally clear that $M$ has a natural foliation by surfaces which are orthogonal to the fibres of the Seifert fibration, namely the images of the planes $E^{2} \times$ point. If $M$ is closed, and so finitely covered by the 3-torus, it is clear that each leaf of the foliation of $M$ is covered by a torus and so must be a torus or Klein bottle. Now it follows that our foliation of $M$ gives $M$ the structure of 
a bundle over a 1-dimensional orbifold. If the base orbifold is a circle, then $M$ is a bundle over the circle with fibre the torus or Klein bottle. We can understand the glueing map of this bundle as follows. There is a natural action of $\mathbb{R}$ on $E^{2} \times E^{1}$ by translating the second factor and this commutes with the action of all elements of $G$. Hence it descends to an action of $\mathbb{R}$ on $M$. Clearly this action preserves the structure of $M$ as a bundle over $S^{1}$ with fibre $F$. Let $t$ be the smallest positive number which leaves each fibre of $M$ invariant, and let $\phi_{t}$ be the diffeomorphism of a fibre $F$ so induced. Then $\phi_{t}$ is a glueing map for the bundle $M$. Now we know that $M$ is also a Seifert fibre space and this action of $\mathbb{R}$ leaves invariant each circle of the Seifert fibration. In particular, it follows that the glueing map $\phi_{t}$ of the bundle $M$ is periodic.

I would like to end this section by briefly discussing the classification of the closed manifolds obtained as quotients of $E^{3}$ by groups of isometries acting freely. These are precisely the closed flat 3-manifolds. We have just seen that any such manifold $M$ is a Seifert fibre space and I will discuss the classification from this point of view. Dunbar [7] has classified all the fibred compact orbifolds obtained as quotients of $E^{3}$ by groups of isometries.

There are seventeen closed 2-dimensional Euclidean orbifolds corresponding to the seventeen discrete groups of isometries of $E^{2}$ with compact quotient, the

TABLE 4.4

THE SEVENTEEN CLOSED 2-DIMENSIONAL EUCLIDEAN ORBIFOLDS

The integers in brackets specify the cone angles. Thus $(n)$ denotes a cone angle $2 \pi / n$.

Note that all boundary curves are reflector curves.

\begin{tabular}{cccc}
\hline $\begin{array}{c}\text { Underlying } \\
\text { surface } X\end{array}$ & Cone points & $\begin{array}{c}\text { Number of Seifert bundles } \\
\text { over } X \text { with } e=0\end{array}$ & $\begin{array}{c}\text { Number with orientable } \\
\text { total space }\end{array}$ \\
\hline Torus & & 3 & 1 \\
Klein bottle & $(2,2,2,2)$ & 5 & 1 \\
$S^{2}$ & $(2,4,4)$ & 1 & 1 \\
$S^{2}$ & $(2,3,6)$ & 1 & 1 \\
$S^{2}$ & $(3,3,3)$ & 1 & 1 \\
$S^{2}$ & $(2,2)$ & 1 & 1 \\
$P^{2}$ & & 2 & 1 \\
Annulus & 2 & 0 \\
Moebius band & $(2,2)$ & 2 & 0 \\
$D^{2}$ & 1 & 0 \\
\hline
\end{tabular}

The remaining seven orbifolds all have corner reflectors and have underlying surface $D^{2}$.

In the first column, an integer $(n)$ specifies a cone angle of $2 \pi / n$. In the second column, an integer $(n)$ specifies an angle of $\pi / n$.

Cone points
Corner reflectors

(2)

$(2,2,2,2)$

$(2,4,4)$

$(2,3,6)$

$(3,3,3)$
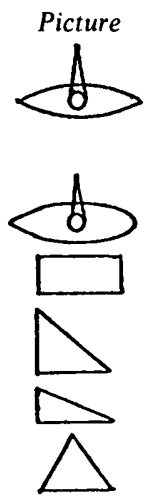
wallpaper groups. See Table 4.4. Seven of these have corner reflectors and so cannot be the base of a Seifert bundle. This leaves ten orbifolds each of which is the base of at least one Seifert bundle with $e=0$, giving a total of nineteen Seifert bundles. See Table 4.4. Briefly, the explanation for the number of Seifert bundles over each orbifold shown in Table 4.4 goes as follows.

A Seifert bundle over $S^{2}(2,2,2,2)$ must have Seifert invariants $(2,1)$ for each critical fibre. The bundle over $S^{2}$ minus four 2-discs centered on the four cone points must be trivial when restricted to each boundary curve and so is trivial. Finally, as we want $e$ to be zero, and as we are in the situation where $b$ is an integer, we see that the bundle is unique. If one considers Seifert bundles over $S^{2}(2,4,4)$, it is again true that the bundle over $S^{2}$ minus three 2-discs centered on the cone points is trivial so that $b$ is an integer and $e=b+\frac{1}{2}+\frac{1}{4} a_{1}+\frac{1}{4} a_{2}$, where $(2,1),\left(4, a_{1}\right)$ and $\left(4, a_{2}\right)$ are the Seifert invariants of the critical fibres. Now $b$ is an integer and we want $e=0$, so the only possibility is $a_{1}=a_{2}=1$ or $a_{1}=a_{2}=3$. The second bundle is isomorphic to the first by an isomorphism reversing fibre orientation.

Similar discussions apply to $S^{2}(3,3,3)$ and $S^{2}(2,3,6)$. There are two Seifert bundles over $P^{2}(2,2)$ with $e=0$, one with orientable total space. Note that there is only one bundle over $P^{2}(2,2)$ with non-orientable total space because, in this case, $b$ is automatically zero in the presence of a Seifert invariant $(2,1)$. The discussion of the classification of Seifert bundles in $\S 3$ shows that reflector curves can be ignored, when deciding how many Seifert bundles there are with a given base. This is why there are two Seifert bundles over the annulus and Moebius band and one over $D^{2}(2,2)$.

Finally, there are three circle bundles over the torus with $e=0$. One has orientable total space, the 3-torus, and the other two are distinguished by the value of the invariant $b$. Also there are five circle bundles over the Klein bottle with $e=0$. One has orientable total space and the other four fall into pairs distinguished only by the value of $b$.

Each of these nineteen Seifert bundles arises naturally as the quotient of $E^{3}$ by some discrete group of isometries acting freely (see $\S 5$ ), but some groups can give rise to more than one bundle if they leave invariant more than one direction in $E^{3}$. The final count of closed flat 3-manifolds is that there are ten altogether with six being orientable. Thus of the seven Seifert bundles in Table 4.4 with orientable total space only two give homeomorphic manifolds. These have base space the Klein bottle and $S^{2}(2,2,2,2)$. The easiest way to see the homeomorphism is to consider the discrete group $G$ of isometries of $E^{3}$ generated by unit translations along the $x$ and $y$-axes of $E^{3}$ and a screw motion along the $z$-axis of $E^{3}$ with rotation angle $\pi$. Note that $G$ contains screw motions along lines parallel to the $z$-axis, one for each integer point in the $x y$-plane. The foliation of $E^{3}$ by lines parallel to the $z$-axis gives $E^{3} / G$ the structure of a Seifert bundle over $S^{2}(2,2,2,2)$ and the foliation by lines parallel to the $x$-axis (for example) gives $E^{3} / G$ the structure of a circle bundle over the Klein bottle. One can also give a cruder construction working directly with the quotient manifolds which uses the fact that the Klein bottle is a Seifert fibre space over the interval with two critical fibres corresponding to the endpoints. This fact was used in $\S 3$.

The remaining twelve Seifert bundles with non-orientable total space yield only four distinct manifolds, which are the total spaces of the four circle bundles over the Klein bottle. Each manifold has three Seifert bundle structures corresponding to three different directions in $E^{3}$ left invariant by the action of its fundamental group. 
For example, let $G$ be the discrete group of isometries of $E^{3}$ generated by translations along the $y$ and $z$-axes of $E^{3}$ and a third isometry which is the composite of a translation along the $x$-axis with reflexion in the $x y$-plane. The foliation of $E^{3}$ by lines parallel to the $y$-axis gives $E^{3} / G$ the structure of a trivial circle bundle over the Klein bottle. The lines parallel to the $z$-axis make $E^{3} / G$ a circle bundle over the torus and the lines parallel to the $x$-axis make $E^{3} / G$ a Seifert fibre space over the annulus. We leave the reader to construct the required isometry groups in the other cases or find them in [46] or [75].

In conclusion, there are ten flat closed 3-manifolds and these are precisely the manifolds which admit a Seifert fibre space structure with $\chi$ and $e$ both equal to zero. Each of these manifolds is finitely covered by the 3-torus. Conversely, it is known that any manifold $M$ finitely covered by the 3-torus is a Seifert fibre space. For $\pi_{1}(M)$ is a finite torsion free extension of $\mathbb{Z} \times \mathbb{Z} \times \mathbb{Z}$ and so is isomorphic [75] to a crystallographic group $\Gamma$. As $\Gamma$ is torsion free, it acts freely on $E^{3}$ yielding a flat manifold $N=E^{3} / \Gamma$. Now we use the fact, discussed after Theorem 4.3 , that $N$ admits the structure of a bundle over a 1-dimensional orbifold. Thus $N$ is either a bundle over the circle or the union of two twisted $I$-bundles. It follows that $N$ is Haken. This means that $N$ is compact and $P^{2}$-irreducible and contains a 2 -sided surface $F$, not $S^{2}$, such that $\pi_{1}(F)$ injects into $\pi_{1}(N)$. (Simply take $F$ to be a fibre when $N$ is a bundle over $S^{1}$, or take $F$ to be the intersection of the two $I$-bundles.) Thus we have two closed 3-manifolds $M$ and $N$ with isomorphic fundamental groups and $N$ is Haken. A famous theorem of Waldhausen [74], extended by Heil [20] to the non-orientable case, shows that $M$ and $N$ are homeomorphic.

The geometry of $H^{3}$. The basic properties of hyperbolic space $H^{3}$ can be developed exactly along the lines which I used for $H^{2}$ in $\$ 1$. One starts with upper half 3-space $\mathbb{R}_{+}^{3}=\left\{(x, y, z) \in \mathbb{R}^{3}: z>0\right\}$ and the formula $d s^{2}=\frac{1}{z^{2}}\left(d x^{2}+d y^{2}+d z^{2}\right)$ to assign a length to any smooth path in $\mathbb{R}_{+}^{3}$ and hence define a metric on $\mathbb{R}_{+}^{3}$. One checks that vertical straight lines are geodesics in this new metric. One also checks that inversion of $\mathbb{R}^{3}$ in a sphere with centre on the $x y$-plane defines an isometry of $H^{3}$. Now one can show that the geodesics of $H^{3}$ are exactly the vertical straight lines and arcs of circles which meet the $x y$-plane orthogonally. One can also show that the full isometry group of $H^{3}$ is generated by reflexions, which are simply the above inversions (including the reflexions in vertical planes). Clearly an isometry of $H^{3}$ is determined by its restriction to the "2-sphere at infinity" consisting of $\mathbb{C} \cup\{\infty\}$, where we identify the $x y$-plane with $\mathbb{C}$.

The group of orientation preserving isometries of $H^{3}$ can be identified with the group of Moebius transformations of $\mathbb{C} \cup\{\infty\}$. Recall that a Moebius transformation of $\mathbb{C} \cup\{\infty\}$ is a map of the form $z \rightarrow \frac{a z+b}{c z+d}$, where $a, b, c, d \in \mathbb{C}$ and $a d-b c \neq 0$. The group of these transformations is naturally isomorphic to $\operatorname{PSL}(2, \mathbb{C})$. We identify the point $(x, y, z)$ of $\mathbb{R}_{+}^{3}$ with the quaternion $x+y i+z j$. The $2 \times 2$ complex matrix $\left(\begin{array}{ll}a & b \\ c & d\end{array}\right)$ acts on $\mathbb{R}_{+}^{3}$, extending its natural action on $\mathbb{C} \cup\{\infty\}$, by the formula $w \rightarrow(a w+b)(c w+d)^{-1}$, where $w$ is a quaternion of the form $x+y i+z j, z>0$. One can check that this yields all orientation preserving isometries of $H^{3}$. 
For most of the basic facts about $H^{3}$, the reader is referred to Thurston [66]. See also Milnor [39]. However, we will need a few results about $H^{3}$ for use later in this article and I shall briefly discuss them here.

It follows at once from the identification of $\operatorname{PSL}(2, \mathbb{C})$ with the group of orientation preserving isometries of $H^{3}$, that each such isometry fixes one or two points of the sphere at infinity. The geometric description of these two cases is as follows. If $\alpha$ is an orientation preserving isometry of $H^{3}$ fixing two points $x$ and $y$ at infinity, it is called hyperbolic. Then $\alpha$ is a screw motion whose invariant axis is the geodesic joining $x$ and $y$. As for $E^{3}$, a rotation is a special case of this. If $\alpha$ fixes a single point at infinity, it is called parabolic as for $H^{2}$. We can conjugate $\alpha$ in $\operatorname{PSL}(2, \mathbb{C})$ so that this fixed point is $\infty$. Now $\alpha$ is of the form $w \rightarrow w+b$, and the group of all parabolic isometries of $H^{3}$ fixing $\propto$ is clearly isomorphic to $\mathbb{R}^{2}$.

As in $\$ 2$, it is now easy to show that isometries of $H^{3}$ rarely commute. The precise result we need is the following. If $\alpha$ is an isometry of $H^{3}$, let fix $(\alpha)$ denote the set of points on the sphere at infinity which are fixed by $\alpha$.

LEMMA 4.5. (i) If $\alpha$ and $\beta$ are two non-trivial orientation preserving isometries of $H^{3}$, then $\alpha$ and $\beta$ commute if and only if $\operatorname{fix}(\alpha)=\mathrm{fix}(\beta)$.

(ii) If $\alpha$ is a non-trivial orientation preserving isometry of $H^{3}$, then the group $C(\alpha)$ of all orientation preserving isometries which commute with $\alpha$ is abelian and isomorphic to $\mathbb{R}^{2}$ or $S^{1} \times \mathbb{R}$.

The proof is essentially the same as the proof of Lemma 1.10. An immediate corollary of this which we will need later is the following.

COROllary 4.6. Let $M^{3}$ be a closed 3-manifold with a hyperbolic structure. Then $\pi_{1}(M)$ cannot contain a subgroup isomorphic to $\mathbb{Z} \times \mathbb{Z}$.

Proof. If $M$ has a hyperbolic structure then $\pi_{1}(M)$ acts freely and discretely on $H^{3}$ as a group of isometries. If $\pi_{1}(M)$ contains a subgroup $G$ isomorphic to $\mathbb{Z} \times \mathbb{Z}$, the preceding lemma shows that $G$ consists of parabolic isometries of $H^{3}$ fixing a single point on the sphere at infinity. By conjugating $\pi_{1}(M)$ in $\operatorname{PSL}(2, \mathbb{C})$, we can suppose that $G$ fixes $\infty$. Let $g$ be an element of $G$ and suppose that $g$ is given by the formula $g(w)=w+b$, where $w$ is a quaternion of the form $x+y i+z j$ and $b$ is a complex number. Then the hyperbolic distance of $g(w)$ from $w$ is clearly less than $|b| / z$, because the hyperbolic metric is given by $d s^{2}=\frac{1}{z^{2}}\left(d x^{2}+d y^{2}+d z^{2}\right)$. Hence, by choosing $z$ large, we see that $g$ moves points of $H^{3}$ arbitrarily small distances. Equivalently, $M$ contains arbitrarily short essential loops. However, a closed Riemannian manifold $M$ cannot contain arbitrarily short essential loops, for there exists a number $d>0$, depending on $M$, such that any metric ball in $M$ of radius $d$ is homeomorphic to a genuine ball. Thus any loop in $M$ of length less than $d$ lies in such a ball and is contractible. This contradiction completes the proof of Corollary 4.6.

The geometry of $S^{3}$. We will think of $S^{3}$ as the unit sphere in $\mathbb{R}^{4}$, as the group of unit quaternions or as the set of ordered pairs $\left(z_{1}, z_{2}\right)$ of complex numbers with $\left|z_{1}\right|^{2}+\left|z_{2}\right|^{2}=1$, using whichever point of view seems convenient. The pair $\left(z_{1}, z_{2}\right)$ of complex numbers is to be identified with the quaternion $z_{1}+z_{2} j$. 
With the induced metric from the standard Euclidean metric on $\mathbb{R}^{4}$, the isometry group of $S^{3}$ is the orthogonal group $\mathrm{O}(4)$. The geodesics can be nicely described as follows. A path $l$ on $S^{3}$ is a geodesic if and only if there is a 2-dimensional plane $\Pi$ in $\mathbb{R}^{4}$ passing through the origin such that $l \subset \Pi \cap S^{3}$. Of course, $\Pi \cap S^{3}$ is always a circle, so that, as for $S^{2}$, all the geodesics are circles. If one assumes the basic existence and local uniqueness results about geodesics on any complete Riemannian manifold, this fact is very easy to see. For let $a$ and $b$ be points of $S^{3}$ close enough so that there is a unique shortest geodesic arc $l$ from $a$ to $b$. Let $\Pi$ denote the 2-plane in $\mathbb{R}^{4}$ through $a, b$ and the origin, and let $\Sigma$ denote any 3-plane in $\mathbb{R}^{4}$ containing $\Pi$. Then reflexion of $\mathbb{R}^{4}$ in $\Sigma$ induces an isometry of $S^{3}$ which fixes all points of $\Sigma$. In particular $a$ and $b$ are fixed so that $l$ must be left invariant. It follows that $l$ lies in $\Sigma$. As this holds for all 3-planes containing $\Pi$, it follows that $l$ lies in $\Pi$ as required.

Now think of $S^{3}$ as the group of unit quaternions. Clearly -1 is a central element in this group and it is easy to show that 1 and -1 together constitute the centre $C$ of $S^{3}$. (For example, if $a+b i+c j+d k$ commutes with $i$, then $c=d=0$.) The quotient of $S^{3}$ by $C$ can be identified with SO (3), the group of all orthogonal real $3 \times 3$ matrices in the following way. We define $\psi: S^{3} \rightarrow \mathrm{SO}(4)$ by letting $\psi(q)$ be the isometry of $S^{3}$ sending $x$ to $q x q^{-1}$. Note that this map is an isometry of $S^{3}$ as left and right multiplication of $S^{3}$ by an element of $S^{3}$ is always an isometry. Clearly the kernel of $\psi$ is exactly the centre $C$ of $S^{3}$ and the image of $\psi$ lies in the subgroup of $\mathrm{SO}$ (4) fixing 1 , which can be naturally identified with $\mathrm{SO}(3)$. As $S^{3}$ is 3-dimensional and $\psi$ has finite kernel, the image of $\psi$ must be a 3-dimensional subgroup of $\mathrm{SO}$ (3). As $\mathrm{SO}(3)$ is a connected 3-dimensional group, $\psi$ must have image $\mathrm{SO}(3)$ as claimed.

We have seen in $\S 3$ that $S^{3}$ can be Seifert fibred in infinitely many different ways. In fact, all these fibrations occur very naturally. The most well-known Seifert fibration of $S^{3}$ is a fibration in the ordinary sense. It is the Hopf fibration which expresses $S^{3}$ as a circle bundle over $S^{2}$. To describe this, we think of $S^{3}$ as pairs of complex numbers and think of $S^{2}$ as $\mathbb{C} \cup\{\infty\}$. Then the Hopf map $h: S^{3} \rightarrow S^{2}$ sends $\left(z_{1}, z_{2}\right)$ to $z_{1} / z_{2}$. This is a bundle whose typical fibre $h^{-1}(\lambda)$ is the circle in $S^{3}$ given by $z_{1}=\lambda z_{2}$ where $\lambda \in \mathbb{C} \cup\{\infty\}$. Each such circle is clearly a geodesic of $S^{3}$.

It is equally easy to describe the other Seifert fibrations of $S^{3}$ over the orbifolds $S^{2}(p, q)$. (For convenience, I shall use $S^{2}(p, 1)$ to denote $S^{2}(p)$ in this discussion.) One again has a map $S^{3} \rightarrow S^{2}$, but the map $h_{p, q}$ is defined by $h_{p, q}\left(z_{1}, z_{2}\right)=z_{1}^{p} / z_{2}^{q}$ where $p$ and $q$ are coprime. This map expresses $S^{3}$ as a Seifert bundle over $S^{2}(p, q)$ with at most two critical fibres whose (unnormalised) orbit invariants are $(p, q)$ and $(q, p)$. In these examples, the fibres are not geodesics of $S^{3}$.

Now consider the action of $S^{3}$ on itself by right multiplication of quaternions. This is certainly a group of isometries of $S^{3}$ though, of course, it does not constitute all of SO (4). A simple calculation will show that this action preserves the Hopf fibration. We consider right multiplication by a fixed unit quaternion $w_{1}+w_{2} j$ where $w_{1}, w_{2} \in \mathbb{C}$ and we denote quaternions by pairs of complex numbers. Then

$$
\left(z_{1}, z_{2}\right)\left(w_{1}, w_{2}\right)=\left(z_{1} w_{1}-z_{2} \bar{w}_{2}, z_{2} \bar{w}_{1}+z_{1} w_{2}\right) \text {. }
$$

Hence the circle $z_{1} / z_{2}=\lambda$ is transformed to the circle $z_{1} / z_{2}=\frac{\lambda w_{1}-\bar{w}_{2}}{\bar{w}_{1}+\lambda w_{2}}$ which is another fibre in the Hopf fibration. Note that this induces a Moebius transformation with determinant 1 of $S^{2}=\mathbb{C} \cup\{\infty\}$. This transformation can only be the identity if the quaternion $w_{1}+w_{2} j$ is 1 or -1 . As the action on $S^{3}$ is a free action, we see that any finite subgroup $G$ of $S^{3}$ acts freely on $S^{3}$ by right multiplication and the quotient 
manifold $M$ inherits a natural Seifert fibration. The induced action of $G$ on $S^{2}=\mathbb{C} \cup\{\infty\}$, the base surface of the Hopf fibration, has just been described as being by Moebius transformations with determinant 1 , and so in particular is orientation preserving. Thus $M$ has an orientable base orbifold.

The other Seifert fibrations are not preserved by all right multiplications. However, right multiplication of $S^{3}$ by the unit complex numbers $S^{1}$, which we identify with the circle $z_{2}=0$ in $S^{3}$, does preserve all these fibrations. This is because $\left(z_{1}, z_{2}\right)\left(w_{1}, 0\right)=\left(z_{1} w_{1}, z_{2} \bar{w}_{1}\right)$ so that the circle $z_{1}^{p} / z_{2}^{q}=\lambda$ is transformed to the circle $z_{1}^{p} / z_{2}^{q}=\lambda w_{1}^{p} / \bar{w}_{1}^{q}$ by right multiplication by $\left(w_{1}, 0\right)$. As before, the induced action on $S^{2}$ can only be the identity if $w_{1}$ is 1 or -1 . Thus a finite, and hence cyclic, subgroup of $S^{1}$ acts freely on $S^{3}$ preserving all the Seifert fibiations so that one obtains quotient manifolds with infinitely many non-isomorphic Seifert bundle structures. These quotients are the lens spaces $L(n, 1), n \geqslant 2$.

If one considers left multiplication instead one finds that none of the Seifert fibrations described above is preserved by all left multiplications. However, left multiplication by the unit complex numbers $S^{1}$ (given by $z_{2}=0$ again), not only preserves all the above Seifert fibrations, but actually leaves each fibre of the Hopf fibration invariant. This is because $\left(w_{1}, 0\right)\left(z_{1}, z_{2}\right)=\left(w_{1} z_{1}, w_{1} z_{2}\right)$.

It is also true that left multiplication of $S^{3}$ by a unit quaternion of the form $\left(0, w_{2}\right)$ preserves the Hopf fibration as $\left(0, w_{2}\right)\left(z_{1}, z_{2}\right)=\left(-w_{2} \bar{z}_{2}, w_{2} \bar{z}_{1}\right)$. Note that the induced action on the base surface $S^{2}=\mathbb{C} \cup\{\infty\}$ of the Hopf fibration is $\lambda \mapsto-1 / \bar{\lambda}$ which is an orientation reversing free involution and so has quotient $P^{2}$. The set of all unit quaternions of the form $\left(0, w_{2}\right)$ together with those of the form $\left(w_{1}, 0\right)$ is a subgroup $\Sigma$ of $S^{3}$ with two components. The structure of $\Sigma$ is best described by observing that under the projection map $\psi: S^{3} \rightarrow \mathrm{SO}(3)$ described earlier, the image of $\Sigma$ is isomorphic to $\mathrm{O}(2)$. The projection $\Sigma \rightarrow \mathrm{O}(2)$ is a double covering on each component. In particular, one can show that a finite subgroup of $\Sigma$ must be cyclic or dihedral or generalized quaternion.

Thus we have free actions of cyclic and dihedral groups on $S^{3}$ given by left multiplication as described above and so preserving the Hopf fibration. The induced action on the base surface $S^{2}$ is trivial or contains a single orientation reversing free involution so that the quotient space inherits the structure of a circle bundle over $S^{2}$ or $P^{2}$.

One can find actions of finite groups on $S^{3}$ which preserve the Hopf fibration and are not obtained simply by left or right multiplication of $S^{3}$ as follows. Let $\Gamma$ be a finite subgroup of $S^{3}$ acting by right multiplication of $S^{3}$ and let $K$ be a finite subgroup of $\Sigma$ acting by left multiplication on $S^{3}$. As these actions commute, we have an action of $K \times \Gamma$ on $S^{3}$ by isometries. (This action need not be effective as both $K$ and $\Gamma$ could contain -1 and $(-1,-1)$ would act trivially.) As $K$ is a subgroup of $\Sigma$, the action of $k \times \Gamma$ must preserve the Hopf fibration of $S^{3}$.

Note that the asymmetry between left and right multiplication is more apparent than real. It is caused by our choices of Seifert fibrations. One can clearly reverse the roles of left and right multiplication by applying inversion of quaternions to $S^{3}$. The inverse of the quaternion $\left(z_{1}, z_{2}\right)$ is $\left(\bar{z}_{1},-z_{2}\right)$ and the isometry $\left(z_{1}, z_{2}\right) \rightarrow\left(\bar{z}_{1},-z_{2}\right)$ of $S^{3}$ sends the Hopf fibres $z_{1} / z_{2}=\lambda$ to the circles given by $\bar{z}_{1} / z_{2}=-\lambda$. These are the fibres of the map $\bar{h}: S^{3} \rightarrow S^{2}$ given by $\bar{h}\left(z_{1}, z_{2}\right)=\bar{z}_{1} / z_{2}$. Similarly one has Seifert fibrations given by $\bar{h}_{p, q}\left(z_{1}, z_{2}\right)=\bar{z}_{1}^{p} / z_{2}^{q}$.

It is a fact, which we shall see shortly, that any free action of a finite group of isometries of $S^{3}$ can be described as above. In particular, any 3-manifold with a 
geometric structure modelled on $S^{3}$ is a Seifert fibre space. Note that such a manifold must be orientable, as any orientation reversing homeomorphism of $S^{3}$ has a fixed point. This can be proved by showing that the Lefschetz number of such a homeomorphism is non-zero. Thus the classification of these manifolds is equivalent to classifying the finite subgroups of SO (4) which act freely on $S^{3}$. These groups were classified by Hopf [24] and I will discuss the complete result. See also [46] and [75].

An important fact for the classification is an amazing isomorphism between SO (4) and a quotient of $S^{3} \times S^{3}$ by $\mathbb{Z}_{2}$. Recall that $S^{3}$ acts on itself by left and right multiplication, that both actions are by isometries and that both actions commute. Thus we have a homomorphism $\phi: S^{3} \times S^{3} \rightarrow \mathrm{SO}(4)$ where $\phi\left(q_{1}, q_{2}\right)$ is the isometry of $S^{3}$ sending $x$ to $q_{1} x q_{2}^{-1}$. If $\left(q_{1}, q_{2}\right)$ lies in the kernel of $\phi$, then $x=q_{1} x q_{2}^{-1}$, for all $x$ in $S^{3}$. Putting $x=1$ shows that $q_{1}=q_{2}$. Hence $q_{1}$ must be central in $S^{3}$. As 1 and -1 are the only central elements of $S^{3}$, we see that the kernel of $\phi$ has order two and that its only non-trivial element is $(-1,-1)$. It follows that the image of $\phi$ is a 6-dimensional subgroup of $\mathrm{SO}$ (4). As $\mathrm{SO}(4)$ is 6-dimensional and connected, $\phi$ must be surjective. Thus $\phi$ induces an isomorphism $\bar{\phi}:\left(S^{3} \times S^{3}\right) / \mathbb{Z}_{2} \rightarrow \mathrm{SO}(4)$ as stated.

Now let $\alpha$ be an orientation preserving isometry of $S^{3}$. Thus $\alpha(x)=q_{1} x q_{2}^{-1}$ for some unit quaternions $q_{1}, q_{2}$. If $\alpha$ fixes $x$, then $x=q_{1} x q_{2}^{-1}$ and so $q_{2}=x^{-1} q_{1} x$. Conversely, if $q_{1}$ and $q_{2}$ are conjugate in $S^{3}$, then $\alpha$ has a fixed point. Thus $\alpha$ has a fixed point if and only if $q_{1}$ and $q_{2}$ are conjugate. This is what allows one to describe all the finite groups of isometries of $S^{3}$ which act freely.

First we need some remarks on conjugacy of quaternions. Recall that the map $\psi: S^{3} \rightarrow \mathrm{SO}(3)$, where $\psi(q)$ is the isometry of $S^{3}$ sending $x$ to $q x q^{-1}$, is a surjection. Hence the action of $S^{3}$ on itself by conjugation has two orbits which are single points and all other orbits are 2 -spheres centered on 1 (or -1 ). As each such 2-sphere must meet the circle $S^{1}$ of unit complex numbers in $S^{3}$, we see that any quaternion $x$ is conjugate in $S^{3}$ to a unit complex number. A nice consequence which we will use shortly is that all unit quaternions of order 4 are conjugate in $S^{3}$. For any such quaternion is conjugate to a unit complex number of order 4 which must be $i$ or $-i$, and $i$ and $-i$ are conjugate as $j^{-1} i j=-i$. Note also that it follows from the above that two unit quaternions are conjugate if and only if they have the same real part. Now we can prove the following fact.

LEMMA 4.7. If $\Gamma$ is a subgroup of $\mathrm{SO}(4)$ of order two which acts freely on $S^{3}$, then the elements of $\Gamma$ are $I$ and $-I$, where I denotes the identity $4 \times 4$ matrix.

Remark. It follows that if $G$ is a finite subgroup of $\mathrm{SO}(4)$ which acts freely on $S^{3}$, then either $G$ has odd order or $G$ contains exactly one element of order 2 , which is $-I$.

Proof of Lemma 4.7. Let the non-trivial element $\alpha$ of $\Gamma$ be the isometry $x \rightarrow q_{1} x q_{2}^{-1}$ of $S^{3}$. As this isometry has order two, we know that $x=q_{1}^{2} x q_{2}^{-2}$, for all unit quaternions $x$. As before, it follows that $q_{1}^{2}=q_{2}^{2}$ and that their common value is 1 or -1 . If the common value is -1 , then $q_{1}$ and $q_{2}$ each have order four. Hence, by the paragraph preceding Lemma 4.7, $q_{1}$ and $q_{2}$ are conjugate in $S^{3}$. Hence the isometry $\alpha$ has a fixed point, which is a contradiction. Hence, we must have $q_{1}^{2}=q_{2}^{2}=1$. Hence each of $q_{1}$ and $q_{2}$ equals 1 or -1 . If $q_{1}=q_{2}$, then $\alpha$ is trivial which is again impossible. Hence one of $q_{1}$ and $q_{2}$ equals 1 and the other equals -1 and $\alpha$ is the isometry $x \rightarrow-x$ as claimed. 
Recall the surjection $\psi: S^{3} \rightarrow \mathrm{SO}(3)$ with kernel $C=\{1,-1\}$. Thus $\psi \times \psi: S^{3} \times S^{3} \rightarrow \mathrm{SO}(3) \times \mathrm{SO}(3)$ is a surjection with kernel $C \times C$. Clearly, there is a unique map $p: \mathrm{SO}(4) \rightarrow \mathrm{SO}(3) \times \mathrm{SO}(3)$ such that $\psi \times \psi=p \circ \phi$, and the kernel of $p$ is the subgroup $\{I,-I\}$ of $\mathrm{SO}(4)$. Note that as $\mathrm{SO}(3)$ is centreless, it follows that the centre of SO (4) contains only $I$ and $-I$. I will denote this group of order two by $K$. Now $K$ acts freely on $S^{3}$ with quotient $P^{3}$, so any element of $\mathrm{SO}(4)$ induces a unique isometry of $P^{3}$. Further, the map $p: \mathrm{SO}(4) \rightarrow \mathrm{SO}(3) \times \mathrm{SO}(3)$ can be identified with the natural map $\operatorname{Isom}\left(S^{3}\right) \rightarrow \operatorname{Isom}\left(P^{3}\right)$.

We can also identify $P^{3}$ with the group $\mathrm{SO}(3)$ as $\mathrm{SO}(3)$ is isomorphic to $S^{3} /\{1,-1\}$. The element $\left(u_{1}, u_{2}\right)$ of SO (3) $\times$ SO (3) clearly acts on SO (3) by the map $x \rightarrow u_{1} x u_{2}^{-1}$. As in the case of $S^{3}$, it follows that this isometry of SO (3) has a fixed point if and only if $u_{1}$ and $u_{2}$ are conjugate in SO (3). As conjugacy classes of rotations in $\mathrm{SO}(3)$ are determined by their rotation angles, this is a very convenient criterion. Note that all elements or order two in $\mathrm{SO}(3)$ are conjugate as they are all rotations through $\pi$.

Now let $G$ be a finite subgroup of $S O(4)$ and consider the map $p: \mathrm{SO}(4) \rightarrow \mathrm{SO}(3) \times \mathrm{SO}(3)$ with kernel $K$ as described above. Let $H$ denote $p(G)$. If $G$ has even order, it must contain $K$, by Lemma 4 .7. In this case the quotient $S^{3} / G$ can be constructed by taking $S^{3} / K$ which is $P^{3}$ and then letting $H$ act on $P^{3}$ by isometries. If $G$ acts freely on $S^{3}$, then $H$ must act freely on $P^{3}$. Conveniently, even when $G$ has odd order, $H$ still acts freely on $P^{3}$. To prove this, let $\bar{G}$ denote the group of isometries of $S^{3}$ generated by $G$ and $K$. The elements of $\bar{G}$ are the isometries $x \rightarrow g(x)$ where $g$ is in $G$ together with the isometries $x \rightarrow-g(x)$. If $\bar{G}$ fails to act freely on $S^{3}$, but $G$ does act freely, then there is $g$ in $G$ and $x$ in $S^{3}$ such that $x=-g(x)$. Hence $g^{2}(x)=x$. As $G$ acts freely on $S^{3}$, it follows that $g^{2}$ is the identity, contradicting our hypothesis that $G$ had odd order. We have shown the following.

LEMMA 4.8. If a finite subgroup $G$ of $\mathrm{SO}(4)$ acts freely on $S^{3}$, then the subgroup $H=p(G)$ of $\mathrm{SO}(3) \times \mathrm{SO}(3)$ acts freely on $P^{3}$. Hence $H$ cannot contain a non-trivial element $\left(u_{1}, u_{2}\right)$ with $u_{1}$ conjugate to $u_{2}$ in $\mathrm{SO}(3)$. In particular, $H$ cannot contain an element $\left(u_{1}, u_{2}\right)$ where $u_{1}$ and $u_{2}$ each have order two.

Now we consider in more detail the possibilities for a finite subgroup $H$ of SO (3) $\times \mathrm{SO}(3)$ acting freely on $P^{3}$. Let $H_{1}$ and $H_{2}$ denote the projections of $H$ into the two factors of $\mathrm{SO}(3) \times \mathrm{SO}(3)$. Thus $H$ is a subgroup of $H_{1} \times H_{2}$. Each of $H_{1}$ and $\mathrm{H}_{2}$ is a finite subgroup of $\mathrm{SO}(3)$ and so is cyclic, dihedral or the orientation preserving symmetry group of a regular solid. We will use the standard notation for these groups. The tetrahedral symmetry group is denoted $T$, the octahedral group is denoted $O$ and the icosahedral group is denoted $I$. Note that $T$ is isomorphic to $A_{4}$, that $O$ is isomorphic to $S_{4}$ and $I$ is isomorphic to $A_{5}$. The dihedral group of order $2 n$ is denoted $D_{n}$. Note that all these groups have even order apart from some cyclic groups.

In general, a subgroup of a direct product need not itself be a direct product. I will say that $H$ is a diagonal subgroup of $H_{1} \times H_{2}$ if there are surjections $f_{1}$ and $f_{2}$ from $H_{1}$ and $H_{2}$ respectively to some group $A$, and $H$ is the kernel of the map $H_{1} \times H_{2} \rightarrow A$ whose restriction to $H_{i}$ is $f_{i}$.

THEOREM 4.9. If $H$ is a finite subgroup of $\mathrm{SO}(3) \times \mathrm{SO}(3)$ which acts freely on $P^{3}$, and if $H_{1}$ and $H_{2}$ denote the projections of $H$ into the two factors, then $H_{1}$ or $H_{2}$ is cyclic. 
If one of the factors $H_{1}$ and $H_{2}$ is non-cyclic, then one of the following holds:

(i) $H=H_{1} \times H_{2}$, where $H_{1}$ and $H_{2}$ have coprime order;

(ii) the non-cyclic factor is isomorphic to $T$ and $H$ is a diagonal subgroup of index three in $\mathrm{H}_{1} \times \mathrm{H}_{2}$. The cyclic factor has order $3 n$, where $n$ is odd;

(iii) the non-cyclic factor is isomorphic to $D_{n}$, for $n$ odd, and $H$ is a diagonal subgroup of index two in $H_{1} \times H_{2}$. The cyclic factor has order $2 m$, where $m$ and $n$ are coprime.

If both $H_{1}$ and $H_{2}$ are cyclic, then $H$ is also cyclic.

Remark. If $H$ has odd order, so do $H_{1}$ and $H_{2}$. Hence $H_{1}$ and $H_{2}$ are both cyclic, so that the theorem implies that $H$ is also cyclic.

Proof. Let $H_{i}^{\prime}=H \cap H_{i}$ for $i=1,2$. Thus $H_{i}^{\prime}$ is a normal subgroup of $H_{i}$ and $H /\left(H_{1}^{\prime} \times H_{2}^{\prime}\right) \cong H_{1} / H_{1}^{\prime} \cong H_{2} / H_{2}^{\prime}$.

As $H$ contains $H_{1}^{\prime} \times H_{2}^{\prime}$, Lemma 4.8 shows that $H_{1}^{\prime}$ or $H_{2}^{\prime}$ must have odd order, and hence be cyclic. We will suppose that $H_{1}^{\prime}$ is cyclic of odd order. Suppose that $b$ is an element of order two in $H_{2}$. There is an element $a$ in $H_{1}$ such that $(a, b)$ is in $H$. As $(a, b)^{2}=\left(a^{2}, 1\right)$, we see that $a^{2}$ lies in $H_{1}^{\prime}$ and so has odd order $r$. Hence $a^{r}$ is trivial or has order two. As $(a, b)^{r}=\left(a^{r}, b\right)$, Lemma 4.8 shows that $a^{r}$ cannot have order two. Hence $a^{r}=1$ and so $b$ lies in $H_{2}^{\prime}$. It follows that $H_{2}^{\prime}$ contains every element of order two in $\mathrm{H}_{2}$.

If $H_{2}^{\prime}=H_{2}$, then also $H_{1}^{\prime}=H_{1}$ and so $H_{1}$ is cyclic of odd order and $H=H_{1} \times H_{2}$ as in (i) of the theorem. Lemma 4.8 shows that $H_{1}$ and $H_{2}$ must have coprime order as stated.

If $\mathrm{H}_{2}^{\prime} \neq \mathrm{H}_{2}$, then $\mathrm{H}_{2}$ must be cyclic or isomorphic to $T$. For $O, I$ and any dihedral group are generated by elements of order two. If $H_{2}$ is isomorphic to $T$, then $H_{2}^{\prime}$ must be $\mathbb{Z}_{2} \times \mathbb{Z}_{2}$ and $H_{2} / H_{2}^{\prime}$ is cyclic of order three. Hence $H_{1} / H_{1}^{\prime}$ is also cyclic of order three. As $H_{1}^{\prime}$ has odd order, so does $H_{1}$ and it follows that $H_{1}$ is cyclic. Thus $H$ is a diagonal subgroup of index three in $\mathrm{H}_{1} \times \mathrm{H}_{2}$ as in (ii) of the theorem.

If $H_{2}^{\prime} \neq H_{2}$ and $H_{2}$ is cyclic, then $H_{2} / H_{2}^{\prime}$ is non-trivial and cyclic. Hence $H_{1} / H_{1}^{\prime}$ is non-trivial and cyclic. Hence $H_{1}$ can only be cyclic or dihedral as $T, O$ and $I$ do not have non-trivial cyclic quotients with cyclic kernel. If $H_{1}$ is dihedral, then $H_{1}^{\prime}$ must have index two in $H_{1}$, as $\mathbb{Z}_{2}$ is the only non-trivial cyclic quotient of a dihedral group. We conclude that $H_{1}$ is isomorphic to $D_{n}$, for $n$ odd, and that $H$ is a diagonal subgroup of index two in $H_{1} \times H_{2}$ as in (iii) of the theorem. The coprimeness statement in (iii) again follows from Lemma 4.8 .

In every case, we see that at least one of $H_{1}$ and $H_{2}$ is cyclic, proving the first statement of Theorem 4.9.

Finally, suppose that both $H_{1}$ and $H_{2}$ are cyclic. Then $H$ must be abelian. If $H$ is not cyclic, it must contain a subgroup $E$ isomorphic to $\mathbb{Z}_{p} \times \mathbb{Z}_{p}$ for some prime $p$. As the image of $E$ in $H_{1}$ must be cyclic, the projection map $E \rightarrow H_{1}$ has non-trivial kernel. Thus $E$ contains an element of the form $\left(1, u_{2}\right)$, where $u_{2}$ has order $p$. Similarly, $E$ contains an element of the form $\left(u_{1}, 1\right)$, where $u_{1}$ has order $p$. Hence $H$ contains all elements $\left(u_{1}^{r}, u_{2}^{s}\right)$, and we can choose $r$ and $s$ so that $u_{1}$ and $u_{2}$ are nontrivial rotations through the same angle. This contradicts Lemma 4.8. We deduce that if $H_{1}$ and $H_{2}$ are cyclic, $H$ must also be cyclic which completes the proof of Theorem 4.9. 
The fact that $H_{1}$ or $H_{2}$ is cyclic when $G$ acts freely allows us to prove the following. Recall the surjection $\phi: S^{3} \times S^{3} \rightarrow \mathrm{SO}(4)$ and the inclusion of $S^{1}$, the unit complex numbers in $S^{3}$. Let $\Gamma_{1}$ denote the subgroup $\phi\left(S^{1} \times S^{3}\right)$ in $S O(4)$, and let $\Gamma_{2}$ denote $\phi\left(S^{3} \times S^{1}\right)$ in $\mathrm{SO}(4)$.

THEOREM 4.10. If $G$ is a finite subgroup of $\mathrm{SO}(4)$ acting freely on $S^{3}$, then $G$ is conjugate in $\mathrm{SO}(4)$ to a subgroup of $\Gamma_{1}$ or $\Gamma_{2}$.

REMARK. $\Gamma_{1}$ and $\Gamma_{2}$ are conjugate subgroups of $O(4)$, using the inversion isometry $q \rightarrow q^{-1}$ of $S^{3}$. It follows that any finite group of isometries of $S^{3}$ which acts freely is conjugate in $\mathrm{O}(4)$ to a group which leaves the Hopf fibration invariant.

Proof. We know that $p(G)$ in $\mathrm{SO}(3) \times \mathrm{SO}(3)$ is a subgroup of $H_{1} \times H_{2}$ and that $H_{1}$ or $H_{2}$ is cyclic. Suppose that $H_{1}$ is cyclic. Let $\bar{G}$ denote $\phi^{-1}\left(p^{-1}\left(H_{1} \times H_{2}\right)\right)$, a subgroup of $S^{3} \times S^{3}$. This is also equal to $\psi^{-1}\left(H_{1}\right) \times \psi^{-1}\left(H_{2}\right) \subset \dot{S}^{3} \times S^{3}$, where $\psi: S^{3} \rightarrow$ SO (3). It will suffice to show that $\bar{G}$ is conjugate to a subgroup of $S^{1} \times S^{3}$. For this it suffices to show that $\psi^{-1}\left(H_{1}\right)$ is conjugate to a subgroup of the unit complex numbers $S^{1}$ in $S^{3}$. Let $q$ be an element of $S^{3}$ projecting under $\psi$ to a generator of $H_{1}$. By conjugating in $S^{3}$, we can arrange that $q$ lies in $S^{1}$. Now $\psi^{-1}\left(H_{1}\right)$ is the subgroup of $S^{3}$ consisting of powers of $q$ and of $-q$. Clearly this is a subgroup of $S^{1}$, thus completing the proof of Theorem 4.10 .

We can go further and give a precise description of the possible finite subgroups $G$ of SO (4) which act freely on $S^{3}$. If $\Gamma$ is a finite subgroup of SO (3), we let $\tilde{\Gamma}$ denote the pre-image of $\Gamma$ in $S^{3}$ under $\psi: S^{3} \rightarrow \operatorname{SO}(3)$. Clearly $\tilde{\Gamma}$ is a central extension of $\mathbb{Z}_{2}$ by $\Gamma$. This extension splits only when $\Gamma$ has odd order in which case $\Gamma$ must be cyclic. Recall the map $\phi: S^{3} \times S^{3} \rightarrow \mathrm{SO}(4)$, and that Theorem 4.10 tells us that if $G$ is a finite subgroup of SO(4) acting freely on $S^{3}$, then $G$ is conjugate in $S O(4)$ to a subgroup of $\Gamma_{1}=\phi\left(S^{1} \times S^{3}\right)$ or of $\Gamma_{2}=\phi\left(S^{3} \times S^{1}\right)$. Also recall the map $p: \mathrm{SO}(4) \rightarrow \mathrm{SO}(3) \times \mathrm{SO}(3)$, and the notation $H=p(G)$ and that $H_{1}$ and $H_{2}$ are the projections of $H$ into the two factors.

THEOREM 4.11. Let $G$ be a finite subgroup of $\Gamma_{1}$ acting freely on $S^{3}$. Then one of the following holds:

(a) $G$ is cyclic;

(b) $H_{2}$ is $T, O, I$ or $D_{n}$ and $H_{1}$ is cyclic of order coprime to the order of $H_{2}$. Also $G=\phi\left(\hat{H}_{1} \times \hat{H}_{2}\right)$ which is isomorphic to $H_{1} \times \hat{H}_{2}$;

(c) $H_{2}$ is $T$ and $H_{1}$ is cyclic of order $3 n$, where $n$ is odd. Also $G$ is a diagonal subgroup of index three in $\phi\left(\hat{H}_{1} \times \hat{H}_{2}\right)$;

(d) $H_{2}$ is $D_{n}$, $n$ odd, and $H_{1}$ is cyclic of order $2 m$, where $m$ and $n$ are coprime. Also $G$ is a diagonal subgroup of index two in $\phi\left(\tilde{H}_{1} \times \hat{H}_{2}\right)$.

Proof. If $G$ has even order, then $G$ must contain $-I$, by Lemma 4.7. Thus $G=p^{-1}(H)$. Now Theorem 4.9 shows that if one of $H_{1}$ and $H_{2}$ is non-cyclic, we must have case (b), (c) or (d) above. In case (b), one has $G$ isomorphic to $H_{1} \times \hat{H}_{2}$ precisely because $H_{1}$ and $\widehat{H}_{2}$ have coprime order. If both $H_{1}$ and $H_{2}$ are cyclic, then $H$ is cyclic by Theorem 4.9 again. As $G$ is a central extension of $\mathbb{Z}_{2}$ by $H$, it follows that $G$ is abelian. Now it follows that $G$ is cyclic, for otherwise $G$ contains a subgroup isomorphic to $\mathbb{Z}_{p} \times \mathbb{Z}_{p}$, for some prime $p$. As $H$ is cyclic, this is impossible if $p>2$, 
and it is impossible when $p=2$ by Lemma 4.7. We conclude that if $H_{1}$ and $H_{2}$ are cyclic, then $G$ is also cyclic. Hence Theorem 4.11 holds when $G$ has even order.

If $G$ has odd order, then $G$ is isomorphic to $H$. Hence $H_{1}$ and $H_{2}$ also have odd order and so are cyclic. Now Theorem 4.9 shows that $H$, and hence $G$, is cyclic.

We can summarise the detailed results of the theorem by saying that $G$ is a subgroup of the group $\phi\left(\hat{H}_{1} \times \hat{H}_{2}\right)$ and that $\tilde{H}_{2}$ acts on $S^{3}$ by right multiplication and $\hat{H}_{1}$ acts by left multiplication. We will now describe briefly which of the standard Seifert fibrations of $S^{3}$ are preserved by $G$.

If $\mathrm{H}_{2}$ is neither cyclic nor dihedral, then only the Hopf fibration of $S^{3}$ is preserved by $G$. In this case the quotient 3-manifold $S^{3} / G$ has a unique Seifert bundle structure $\eta$ which is inherited from the Hopf fibration of $S^{3}$. The base orbifold of $\eta$ is $S^{2} / H_{2}$ for, as pointed out earlier, $\hat{H}_{1}$ preserves each fibre of the Hopf fibration and no element of $\tilde{H}_{2}$, except for -1 , does this. The Poincare homology sphere is obtained in this way with $H_{1}$ trivial and $H_{2}=I$.

If $H_{2}$ is cyclic, then we can conjugate $\hat{H}_{2}$ in $S^{3}$ to lie in the unit complex numbers. So we will consider $G$ to be a subgroup of $\phi\left(S^{1} \times S^{1}\right)$. We know that $G$ is cyclic. Now the actions of $\hat{H}_{1}$ and $\hat{H}_{2}$ each preserve all the Seifert fibrations of $S^{3}$ given by the maps $h_{p, q}: S^{3} \rightarrow S^{2}$, where $h_{p, q}\left(z_{1}, z_{2}\right)=z_{1}^{p} / z_{2}^{q}$ and all the Seifert fibrations of $S^{3}$ given by the maps $\bar{h}_{p, q}$ where $\bar{h}_{p, q}\left(z_{1}, z_{2}\right)=\bar{z}_{1}^{p} / z_{2}^{q}$. Hence $G$ acts on $S^{3}$ preserving all these Seifert fibrations. All the induced Seifert bundle structures on $S^{3} / G$ have base orbifold $S^{2}, S^{2}(p)$ or $S^{2}(p, q)$ and so $S^{3} / G$ can be expressed as the union of two fibred solid tori. These quotients are the lens spaces. The lens space $L(p, q)$, where $p$ and $q$ are coprime, is defined to be the quotient of $S^{3}$ by the free action of $\mathbb{Z}_{p}$ given by $\left.\left(z_{1}, z_{2}\right) \rightarrow(\omega)^{q} z_{1}, \omega z_{2}\right)$, where $\left.\omega\right)=e^{2 \pi i / p}$. See [46] for the connection between $p, q$ and the invariants of the Seifert bundle structures on $L(p, q)$.

If $\mathrm{H}_{2}$ is dihedral, then we can conjugate its cyclic normal subgroup of index two so as to lie in the unit complex numbers. The elements of $\hat{H}_{2}$ must now be of the form $\left(w_{1}, 0\right)$ or $\left(0, w_{2}\right)$. In this case, the action of $G$ on $S^{3}$ preserves the Hopf fibration given by $h: S^{3} \rightarrow S^{2}$ where $h\left(z_{1}, z_{2}\right)=z_{1} / z_{2}$ and does not preserve the Seifert fibrations determined by the maps $h_{p, q}$. However, the action of $G$ on $S^{3}$ does preserve the dual Hopf fibration of $S^{3}$ given by $\bar{h}$ where $\bar{h}\left(z_{1}, z_{2}\right)=\bar{z}_{1} / z_{2}$. This corresponds to the fact, pointed out earlier, that left multiplication by a unit quaternion of the form $\left(0, w_{2}\right)$ preserves the Hopf fibration. In this case, $S^{3} / G$ inherits two Seifert fibrations, one, $\eta$, from the Hopf fibration and one, $\bar{\eta}$, from the dual Hopf fibration. The base orbifold of $\eta$ is the orientable orbifold $S^{2} / H_{2}$, which has the form $S^{2}(2,2, n)$, where $H_{2}=D_{n}$. The base orbifold of $\bar{\eta}$ is non-orientable and is of the form $P^{2}(p)$. The orientable double covering of this orbifold, which is of the form $S^{2}(p, p)$ is the quotient $S^{2} / H_{1}$, where $H_{1}$ is $\mathbb{Z}_{p}$. These quotients of $S^{3}$ are the prism manifolds. Each prism manifold is double covered by a lens space.

Apart from prism manifolds and lens spaces, all these quotients of $S^{3}$ have a unique Seifert bundle structure. The prism manifolds admit two Seifert bundle structures and the lens spaces all admit infinitely many Seifert bundle structures. Each of these Seifert bundle structures is inherited from one of the standard Seifert fibrations of $S^{3}$ which have base orbifold $S^{2}, S^{2}(p)$ or $S^{2}(p, q)$. Each of these fibrations of $S^{3}$ also has non-zero Euler number $e$. The naturality of the Euler numbers shows that if $M^{3}$ has a geometric structure modelled on $S^{3}$ and if $\eta$ is one of the induced Seifert bundle structures on $M$ with base orbifold $X$, then $e(\eta) \neq 0$ and $\chi(X)>0$. 
All the quotients of $S^{3}$ admit group actions, which can be described very nicely in terms of the group structure of $S^{3}$. If $G$ is a finite subgroup of SO (4) acting freely on $S^{3}$ we know that $G$ is conjugate in $\mathrm{O}(4)$ to a subgroup of $\Gamma_{1}=\phi\left(S^{1} \times S^{3}\right)$. Hence left multiplication of $S^{3}$ by $S^{1}$, the unit complex numbers, gives a circle action on $S^{3}$ which descends to an action on $S^{3} / G$. In the special case when $H_{1}$ is trivial, then $G$ acts on $S^{3}$ by right multiplication. Hence the action of $S^{3}$ on itself by left multiplication descends to $S^{3} / G$. Thus $S^{3} / G$ admits an action of $S^{3}$ in this case. These manifolds are the only 3-manifolds which admit an effective action of $S^{3}$.

The geometry of $S^{2} \times \mathbb{R}$. This is probably the least interesting but also the simplest of the eight geometries. There are only seven 3-manifolds without boundary, including $S^{2} \times \mathbb{R}$ itself, with geometric structure modelled on $S^{2} \times \mathbb{R}$. The noncompact ones are $S^{2} \times \mathbb{R}$ and the two line bundles over $P^{2}$. The compact ones are the two $S^{2}$-bundles over $S^{1}$, and $P^{2} \times S^{1}$ and $P^{3} \# P^{3}$ where $P^{n}$ denotes real projective space of dimension $n$ and \# denotes connected sum. Note that $P^{3} \# P^{3}$ is the only closed 3-manifold which possesses a geometric structure and is a non-trivial connected sum.

We start by describing groups of isometries of $S^{2} \times \mathbb{R}$ with the above quotients. The isometry group of $S^{2} \times \mathbb{R}$ can be identified with $\operatorname{Isom}\left(S^{2}\right) \times \operatorname{Isom}(\mathbb{R})$. If we take $G$ to be the group of order two generated by the element $(\alpha, \beta) \in \operatorname{Isom}\left(S^{2}\right) \times \operatorname{Isom}(\mathbb{R})$, where $\alpha$ is the antipodal map of $S^{2}$ and $\beta$ is the identity, then $\left(S^{2} \times \mathbb{R}\right) / G$ is $P^{2} \times \mathbb{R}$. If we replace $\beta$ by a reflexion, then $\left(S^{2} \times \mathbb{R}\right) / G$ becomes the non-trivial line bundle over $P^{2}$. If we take $G$ to be the infinite cyclic group generated by $(\alpha, \beta)$ where $\alpha$ is the identity and $\beta$ is a translation, then $\left(S^{2} \times \mathbb{R}\right) / G$ is $S^{2} \times S^{1}$. This quotient is Seifert fibred by circles which are the images of the lines $\{x\} \times \mathbb{R}$ in $S^{2} \times \mathbb{R}$. It is the trivial circle bundle over $S^{2}$. If we replace $\alpha$ by the antipodal map of $S^{2}$, then $\left(S^{2} \times \mathbb{R}\right) / G$ becomes the non-trivial $S^{2}$-bundle over $S^{1}$ which we denote $S^{2} \tilde{x} S^{1}$. Again this is naturally Seifert fibred, this time as a circle bundle over $P^{2}$. If we let $G$ be generated by $\left(\alpha_{1}, \beta_{1}\right)$ and $\left(\alpha_{2}, \beta_{2}\right)$ where $\alpha_{1}$ is the identity, $\beta_{1}$ is a translation, $\alpha_{2}$ is the antipodal map and $\beta_{2}$ is the identity, then $\left(S^{2} \times \mathbb{R}\right) / G$ is $P^{2} \times S^{1}$, which inherits a natural Seifert fibration as trivial circle bundle over $P^{2}$. Finally, if we let $G$ be generated by $\left(\alpha_{1}, \beta_{1}\right)$ and $\left(\alpha_{2}, \beta_{2}\right)$ where $\alpha_{1}$ and $\alpha_{2}$ are each the antipodal map and $\beta_{1}$ and $\beta_{2}$ are distinct reflexions, then $\left(S^{2} \times \mathbb{R}\right) / G$ is the union of two twisted $I$-bundles over $P^{2}$ each with orientable total space and hence is $P^{3} \# P^{3}$. Again there is a natural Seifert fibration as a circle bundle over $P^{2}$.

As in the case of groups acting freely on $E^{3}$, it is clear that each of the above seven manifolds has the structure of a bundle over a 1-dimensional orbifold. For $S^{2} \times \mathbb{R}$ is foliated by the 2-spheres $S^{2} \times\{\mathrm{pt}\}$ and this descends to a foliation of all the quotient manifolds. The base orbifold is $\mathbb{R}$ for $S^{2} \times \mathbb{R}$ and $P^{2} \times \mathbb{R}$, and is a half open interval with one reflector point in the case of the non-trivial line bundle over $P^{2}$. The base is a circle for $S^{2} \times S^{1}, S^{2} \tilde{\times} S^{1}$ and $P^{2} \times S^{1}$ and is a closed interval with two reflector points for $P^{3} \# P^{3}$.

This point of view yields the simplest proof that the above seven manifolds are the only ones which admit a geometric structure modelled on $S^{2} \times \mathbb{R}$. The natural foliation by 2 -spheres of $S^{2} \times \mathbb{R}$ is preserved by all isometries of $S^{2} \times \mathbb{R}$. Hence any manifold $M$ which is the quotient of $S^{2} \times \mathbb{R}$ by a discrete group $G$ of isometries acting freely inherits a foliation by surfaces each of which is a sphere or projective plane. Clearly $M$ is a bundle over a 1 -dimensional orbifold $X$ which is the quotient of $\mathbb{R}$ by 
some group action. The only possibilities for $X$ are those described in the previous paragraph and it follows that $M$ is one of the seven manifolds described previously.

In fact, one can show that these seven 3-manifolds are the only manifolds covered by $S^{2} \times \mathbb{R}$ without any hypothesis on metrics. Here is a sketch of the proof. Let $M$ be a 3-manifold covered by $S^{2} \times \mathbb{R}$. If $\pi_{1}(M)$ is finite then Epstein [9] showed that either $M$ must be the connected sum of a closed orientable manifold with a simply connected manifold or $\pi_{1}(M)$ is $\mathbb{Z}_{2}$ and is carried by a two-sided projective plane in $M$. In the first case, $\pi_{1}(M)$ must be trivial or $\mathbb{Z}_{2}$, as otherwise the universal cover of $M$ would have more than two ends. It follows that $M$ is $S^{2} \times \mathbb{R}$ or the non-trivial line bundle over $P^{2}$. In the second case, it follows that $M$ is $P^{2} \times \mathbb{R}$. Now suppose that $\pi_{1}(M)$ is infinite. Then, using the fact that $S^{2} \times \mathbb{B}$ has two ends, standard methods show that $M$ is compact. Tollefson [71] has shown that the only compact 3-manifolds covered by $S^{2} \times \mathbb{R}$ are the four already described.

We have already seen that the four compact quotients of $S^{2} \times \mathbb{R}$ are Seifert fibre spaces, but as for $E^{3}$, there are two points to watch. Firstly, if $G$ is an arbitrary discrete group of isometries of $S^{2} \times \mathbb{R}$, the quotient need not possess a natural Seifert fibration. For example, let $G$ be generated by a screw motion, i.e. an element $(\alpha, \beta)$ of $\operatorname{Isom}\left(S^{2}\right) \times \operatorname{Isom}(\mathbb{R})$ where $\beta$ is a translation and $\alpha$ is a rotation, and suppose that $\alpha$ has infinite order. Then $\left(S^{2} \times \mathbb{R}\right) / G$ is still $S^{2} \times S^{1}$ but it inherits a foliation by lines not circles. Secondly, two of these four manifolds admit infinitely many Seifert bundle structures. For example, one can obtain $S^{2} \times S^{1}$ as the quotient of $S^{2} \times \mathbb{R}$ by the cyclic group generated by a screw motion whose rotation part can have any specified order. Thus $S^{2} \times S^{1}$ can be regarded as a Seifert fibre space over $S^{2}(n, n)$ for any $n$.

If $M$ is a closed 3-manifold with a geometric structure modelled on $S^{2} \times \mathbb{R}$ and if the natural foliation of $S^{2} \times \mathbb{R}$ by lines descends to a foliation of $M$ by circles, then we have a Seifert bundle structure $\eta$ on $M$. Clearly, the base orbifold $X$ of this bundle is of spherical type, i.e. it is orbifold covered by $S^{2}$, and so $\chi(X)>0$. It is also easy to see that the Euler number $e(\eta)$ is zero. For the hypothesis that the natural foliation on $S^{2} \times \mathbb{R}$ descends to a foliation of $M$ by circles implies that $\pi_{1}(M)$ contains an element $g$ which acts on $S^{2} \times \mathbb{R}$ by a pure translation i.e. it acts trivially on the $S^{2}$-factor and by a translation on the $\mathbb{R}$-factor. Now the quotient of $S^{2} \times \mathbb{P}$ by the cyclic group generated by $g$ is clearly $S^{2} \times S^{1}$ and this gives a finite covering $\hat{M}$ of $M$ whose induced Seifert bundle structure $\tilde{\eta}$ plainly has $e(\tilde{\eta})=0$. The fact that $e(\eta)$ is zero follows from the naturality of the Euler number under coverings.

In fact, the Seifert bundles which arise in this way from the geometry of $S^{2} \times \mathbb{R}$ are precisely those with spherical base orbifold $X$ and with zero Euler number. As in the case of $E^{3}$, one can classify the possible base orbifolds and then classify the Seifert bundles over these orbifolds with zero Euler number. A new point arises here. There are some spherical orbifolds $X$ such that any Seifert bundle over $X$ must have nonzero Euler number. We will now briefly discuss this.

The finite subgroups of $S O(3)$ are trivial, cyclic, dihedral or $\Delta(2,3,3)$, $\Delta(2,3,4), \Delta(2,3,5)$, and the corresponding spherical orbifolds are $S^{2}, S^{2}(n, n)$, $S^{2}(2,2, n), \quad S^{2}(2,3,3), \quad S^{2}(2,3,4)$ and $S^{2}(2,3,5)$. One can now obtain the list of closed orbifolds of spherical type and without corner reflectors which is shown in Table 4.12.

Now consider a Seifert bundle $\eta$ with base $S^{2}(2,2, n), n \geqslant 2$. The circle bundle over $S^{2}$ minus three discs centered at the cone points must be trivial as it is trivial on all three boundary circles. Hence $\eta$ has orientable total space and we have the 
TABLE 4.12

\begin{tabular}{lcl}
\hline $\begin{array}{c}\text { Closed orbifolds of spherical type } \\
\text { and without corner reflectors }\end{array}$ & $\begin{array}{c}\text { Number of Seifert } \\
\text { bundles with } e=0\end{array}$ & \multicolumn{1}{c}{ Total spaces } \\
\hline$S^{2}$ & 1 & $S^{2} \times S^{1}$ \\
$S^{2}(n, n), \quad n \geqslant 2$ & $\infty$ & $S^{2} \times S^{1}$ \\
$S^{2}(2,2, n), \quad n \geqslant 2$ & 0 & \\
$S^{2}(2,3,3)$ & 0 & \\
$S^{2}(2,3,4)$ & 0 & \\
$S^{2}(2,3,5)$ & 0 & $P^{2} \times S^{1}, S^{2} \times S^{1}, P^{3} \# P^{3}$ \\
$P^{2}$ & 3 & $P^{2} \times S^{1}$ \\
$P^{2}(n), \quad n \geqslant 2$ & $\infty$ & $P^{2} \times S^{1}$ \\
$D^{2}$ & 1 & $P^{2} \times S^{1}$ \\
$D^{2}(n), \quad n \geqslant 2$ & $\infty$ & \\
\hline
\end{tabular}

equation $e=b+\frac{1}{2}+\frac{1}{2}+m / n$ for some $m$ coprime to $n$. As $b$ is an integer, it is clear that $e$ cannot be zero. Similarly, there are no Seifert bundles with $e=0$ over $S^{2}(2,3,3), \quad S^{2}(2,3,4)$ or $S^{2}(2,3,5)$. Also there is no Seifert bundle over $P^{2}(n)$ with orientable total space and $e=0$.

Hence the only Seifert bundles we need consider are those with base $S^{2}$, $S^{2}(n, n), P^{2}, P^{2}(n)$ or $D^{2}(n)$. There is only one circle bundle over $S^{2}$ with $e=0$ and this has total space $S^{2} \times S^{1}$. There are many Seifert bundles over $S^{2}(n, n)$ with $e=0$. Any such bundle has orientable total space and Seifert invariants $(n, m),(n, n-m)$ where $m$ and $n$ are coprime. All these bundles have total space $S^{2} \times S^{1}$ also. There is one circle bundle over $P^{2}$ with orientable total space and $e=0$. The total space of this bundle is $P^{3} \# P^{3}$. There are two circle bundles over $P^{2}$ with non-orientable total space which are distinguished by the invariant $b$. The total spaces are $P^{2} \times S^{1}$ and $S^{2} \bar{x} S^{1}$. There is no Seifert bundle over $P^{2}(n)$ with orientable total space and $e=0$. There are many Seifert bundles over $P^{2}(n)$ with non-orientable total space. Each such bundle is determined by its Seifert invariants $(n, m)$ and the invariant $b$ in $\mathbb{Z}_{2}$ (except that $b$ must be zero if $n=2$ ). All these bundles have total space $P^{2} \times S^{1}$. Finally, there are many Seifert bundles over $D^{2}(n)$, and these also have total space $P^{2} \times S^{1}$.

A point which is helpful when deciding which manifold is the total space of one of these Seifert bundles is that the fundamental group of the base orbifold $X$ is a quotient of the fundamental group of the total space $M$. Hence if $\pi_{1}(X)$ is not cyclic, $\pi_{1}(M)$ must be non-cyclic.

We conclude from the above that $S^{2} \times S^{1}$ and $P^{2} \times S^{1}$ admit infinitely many distinct Seifert bundle structures and that $S^{2} \tilde{\times} S^{1}$ and $P^{3} \# P^{3}$ each admit only one such structure.

It is not hard to see that each of these Seifert bundle structures can be obtained naturally by taking a suitable group of isometries of $S^{2} \times \mathbb{R}$.

The geometry of $H^{2} \times \mathbb{R}$. Unlike the previous product $S^{2} \times \mathbb{R}$, there are clearly infinitely many 3-manifolds with geometric structure modelled on $H^{2} \times \mathbb{R}$. For example, the product of any hyperbolic surface with $\mathbb{R}$ or $S^{1}$ has such a structure. However, there are still many similarities.

The isometry group of $H^{2} \times \mathbb{R}$ is naturally isomorphic to $\operatorname{Isom}\left(H^{2}\right) \times \operatorname{Isom}(\mathbb{R})$. As the foliation of $H^{2} \times \mathbb{R}$ by the lines $\{x\} \times \mathbb{R}$ is left invariant by this group, it is clear that this foliation descends to a foliation by lines or circles of any 3-manifold with 
geometric structure modelled on $H^{2} \times \mathbb{R}$. We will show that, in most cases, this induced foliation is by circles and so is a Seifert fibration. Suppose that $G$ is a discrete group of isometries of $H^{2} \times \mathbb{R}$ acting freely with quotient $M$ so that the induced foliation on $M$ is a Seifert fibration. There is a natural projection $G \rightarrow \operatorname{Isom}\left(H^{2}\right)$ with image $\Gamma$ and the base orbifold $X$ of $M$ is clearly $H^{2} / \Gamma$. In particular, if $X$ is compact, then $\chi(X)<0$. One can also show that the Seifert bundle structure $\eta$ induced on $M$ has Euler number $e(\eta)$ equal to zero. In particular, as discussed in $\S 3$, if $M$ is closed then $M$ has a finite covering $\bar{M}$ whose induced Seifert bundle structure $\tilde{\eta}$ is a trivial circle bundle, that is, $\bar{M}$ is isomorphic to $S^{1} \times F$ where $F$ is the base surface of $\tilde{\eta}$.

As for $E^{3}$ and $S^{2} \times \mathbb{R}$, the invariant product structure on $H^{2} \times \mathbb{R}$ also yields a natural foliation of $M$ by surfaces. If $M$ is compact, the fact that $M$ is finitely covered by $S^{1} \times F$ shows that all the leaves of this foliation are compact, so that $M$ also has the structure of a bundle over a 1-dimensional orbifold. If the base is a circle, so that $M$ is a bundle in the usual sense, then the glueing map of this bundle is periodic. This can be proved, exactly as for $E^{3}$, by using the natural action of $\mathbb{R}$ on $H^{2} \times \mathbb{R}$ translating the second factor.

Now I propose to prove some of the above statements. The main result is the following.

THEOREM 4.13. Let $G$ be a discrete group of isometries of $H^{2} \times \mathbb{R}$ which acts freely and has quotient $M$. Then one of the following three conditions holds:

(i) the natural foliation of $H^{2} \times \mathbb{R}$ by lines descends to a Seifert bundle structure on $M$;

(ii) the natural foliation of $H^{2} \times \mathbb{R}$ by lines gives $M$ the structure of a line bundle over some hyperbolic surface;

(iii) the natural foliation of $H^{2} \times \mathbb{R}$ by lines descends to a foliation of $M$ by lines in which each line has non-closed image in $M$. In this case, $G$ must be isomorphic to one of $\mathbb{Z}, \mathbb{Z} \times \mathbb{Z}$ or the Klein bottle group.

Remarks. If case (ii) holds, then $G$ is isomorphic to the fundamental group of some hyperbolic surface. This includes all countable free groups and the trivial group. Clearly case (ii) occurs, for if $X$ is $H^{2} / G$, then the product of this action of $G$ on $H^{2}$ with the identity action on $\mathbb{R}$ gives $M=X \times \mathbb{R}$. The foliation on $M$ in case (iii) will become apparent during the proof.

Proof of Theorem 4.13. We identify the isometry group of $H^{2} \times \mathbb{R}$ with Isom $\left(H^{2}\right) \times \operatorname{Isom}(\mathbb{R})$ in the obvious way and regard the factors as subgroups in the usual way. As $G$ is discrete, $K=G \cap \operatorname{Isom}(\mathbb{R})$ is discrete and so must be $1, \mathbb{Z}_{2}, \mathbb{Z}$ or $D(\infty)$. As $G$ acts freely on $H^{2} \times \mathbb{R}$, it must be torsion free so that $K$ is 1 or $\mathbb{Z}$. In either case, $K$ is normal in $G$ and is the kernel of the projection map $G \rightarrow \operatorname{Isom}\left(H^{2}\right)$. Let $\Gamma$ denote the image of this projection. Then we have the exact sequence

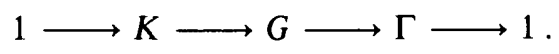

In the case when $K$ is infinite cyclic, each line $\{x\} \times \mathbb{R}$ covers a circle in $\left(H^{2} \times \mathbb{R}\right) / K$, so we clearly have case (i) of the theorem. 
Now we turn to the case when $K$ is trivial. Thus the map $G \rightarrow \Gamma$ is an isomorphism. If $\Gamma$ is a discrete group of isometries of $H^{2}$, it must act freely as it is torsion free. Now it is clear that $M$ is a line bundle over the hyperbolic surface $H^{2} / \Gamma$, so that we have case (ii) of the theorem.

Finally, we consider the most tricky case, when $K$ is trivial and $\Gamma$ is an indiscrete group of isometries of $H^{2}$. This means that we are in case (iii) of the theorem and it remains to show that $G$ is one of the three specified groups. We will show that $G$ has a subgroup of finite index isomorphic to $\mathbb{Z}$ or $\mathbb{Z} \times \mathbb{Z}$. As $G$ is torsion free, this will show that $G$ is isomorphic to one of $\mathbb{Z}, \mathbb{Z} \times \mathbb{Z}$ or the Klein bottle group, as required.

By replacing $G$ by a subgroup of index two, if necessary, we can suppose that $\Gamma$ acts orientation preservingly on $H^{2}$, so that $\Gamma$ is a subgroup of $\operatorname{PSL}(2, \mathbb{R})$. We will now consider the projection map $G \rightarrow \operatorname{Isom}(\mathbb{R})$. Let $L$ denote the kernel of this map. Let $\Lambda$ denote the image of $L$ under the isomorphism $G \rightarrow \Gamma$. As $G$ acts discretely on $H^{2} \times \mathbb{R}$ and $L$ preserves $H^{2} \times\{y\}$, for each $y$ in $\mathbb{R}$, it follows that $\Lambda$ must be a discrete group of isometries of $H^{2}$. Of course, $\Lambda$ is also a normal subgroup of $\Gamma$.

Suppose that $\Lambda$ is non-trivial. Conjugation of $\Lambda$ by each element of $\Gamma$ induces an automorphism of $\Lambda$. As $\Lambda$ is discrete an element of $\Gamma$ suitably close to the identity must commute with $\Lambda$. As $\Gamma$ is indiscrete, there must be a non-trivial element $\gamma$ of $\Gamma$ which centralizes $\Lambda$. Now the centralizer of any non-trivial element in PSL $(2, \mathbb{R})$ is always abelian by Lemma 2.10 , so it follows that $\Lambda$ is abelian. As $\Lambda$ is discrete and torsion free, $\Lambda$ must be infinite cyclic. Hence $\Gamma$ has a subgroup $\Gamma_{1}$ of index at most two which centralizes $\Lambda$. Now Lemma 2.10 shows that $\Gamma_{1}$ is abelian. If $\Gamma_{1}$ consists of hyperbolic isometries there is a unique geodesic $l$ in $H^{2}$ left invariant by $\Gamma_{1}$. If $G_{1}$ denotes the subgroup of $G$ corresponding to $\Gamma_{1}$, we see that $G_{1}$ leaves invariant the plane $l \times \mathbb{R}$ contained in $H^{2} \times \mathbb{R}$. As this plane is isometric to the Euclidean plane and $G_{1}$ must act discretely on it, it follows that $G_{1}$ is $\mathbb{Z}$ or $\mathbb{Z} \times \mathbb{Z}$. If $\Gamma_{1}$ consists of parabolic isometries, then $\Gamma_{1}$ leaves invariant each horocycle of $H^{2}$ centered at its fixed point. (Recall that in the upper half-plane model of $H^{2}$, if we take $\Gamma_{1}$ to fix $\infty$, then $\Gamma_{1}$ consists of horizontal translations and so leaves invariant each line $y=$ constant. These are the horocycles.) We can now apply the same argument taking $l$ to be any one of these horocycles. This completes the proof of case (iii) of the theorem when $\Lambda$ is non-trivial.

Now we consider the case when $\Lambda$ is trivial. Recall that $\Lambda$ is the kernel of the projection map $G \rightarrow \operatorname{Isom}(\mathbb{R})$. The orientation preserving subgroup of $\operatorname{Isom}(\mathbb{R})$ is isomorphic to $\mathbb{R}$ and so is abelian. Hence $G$ has a subgroup $G_{1}$ of index at most two which is abelian. All the arguments of the previous paragraph now apply to show that $G_{1}$ is isomorphic to $\mathbb{Z}$ or $\mathbb{Z} \times \mathbb{Z}$, which completes the proof of Theorem 4.13 .

This theorem shows that if $M$ is a closed 3-manifold which admits a geometric structure modelled on $H^{2} \times \mathbb{R}$ then $M$ has a natural Seifert fibration. For the manifolds in cases (ii) and (iii) of the theorem cannot be closed. As I pointed out at the start of this section, the base orbifold $X$ of $M$ has a hyperbolic structure and so $\chi(X)<0$. It remains to show that the Seifert bundle structure $\eta$ on $M$ has Euler number $e(\eta)$ equal to zero. As discussed in $\S 3, M$ has a finite covering space $\bar{M}$ which is orientable and is a circle bundle $\tilde{\eta}$ over a closed orientable surface $\bar{X}$. The naturality property of the Euler number of a Seifert bundle means that we need only show that $e(\tilde{\eta})$ is zero. Let $\bar{a}_{1}, \bar{b}_{1}, \ldots, \bar{a}_{g}, \bar{b}_{g}$ be a standard set of generators of $\pi_{1}(\bar{X})$, so they satisfy the single relation $\prod_{i=1}^{g}\left[\bar{a}_{i}, \bar{b}_{i}\right]=1$. Let $a_{i}, b_{i}$ be elements of $\tilde{G}$ which 
project to $\bar{a}_{i}$ and $\bar{b}_{i}$ in $\pi_{1}(\tilde{X})$. Then, as discussed in $\S 3, \prod_{i=1}^{g}\left[a_{i}, b_{i}\right]=k^{e}$, where $k$ is a generator of $K$, the kernel of the map $\tilde{G} \rightarrow \pi_{1}(\tilde{X})$, and $e$ is $e(\tilde{\eta})$. Now projection into Isom $(\mathbb{R})$ shows $k^{e}=1$, because each $a_{i}$ and $b_{i}$ projects to a translation of $\mathbb{R}$, by our choice of $\bar{M}$. Hence $e(\bar{\eta})$ is zero, as required. In particular, $\bar{M}$ is homeomorphic to $S^{1} \times \bar{X}$.

The geometry of $\widetilde{\mathrm{SL}_{2} \mathbb{R}}$. The 3-dimensional Lie group of all $2 \times 2$ real matrices with determinant 1 is denoted $\mathrm{SL}_{2} \mathbb{R}$, and $\widetilde{\mathrm{SL}_{2} \mathbb{R}}$ denotes its universal covering. For brevity, I shall omit the $\mathbb{R}$ in the rest of this section and simply write $\mathrm{SL}_{2}$ or $\widetilde{\mathrm{SL}}_{2}$. Of course $\widetilde{\mathrm{SL}}_{2}$ is itself a Lie group and so admits a metric invariant under left multiplication (or right multiplication, but not both). However, for our purposes, there is a more useful way to describe the metric in which we are interested. I will start with a very brief description and then fill in the details.

If $M^{n}$ is a Riemannian manifold, there is a natural Riemannian metric on the tangent bundle $T M$ of $M$, which is $2 n$-dimensional. If $f: M \rightarrow M$ is an isometry, then $d f: T M \rightarrow T M$ is also an isometry. We will use this when $M$ is the hyperbolic plane $H^{2}$. This metric on $T H^{2}$ induces a metric on the unit tangent bundle $U H^{2}$ of $H^{2}$ as this is a submanifold of $T H^{2}$. Now there is a natural identification of $U H^{2}$ with $\mathrm{PSL}_{2} \mathbb{R}$, the orientation preserving isometry group of $H^{2}$, so we have a metric on $\mathrm{PSL}_{2} \mathbb{R}$. Finally $\mathrm{PSL}_{2} \mathbb{R}$ is double covered by $\mathrm{SL}_{2} \mathbb{R}$ and so its universal covering is $\widetilde{\mathrm{SL}}_{2}$. The induced metric on $\widetilde{\mathrm{SL}}_{2}$ is the one in which we are interested. As $U H^{2}$ is a circle bundle over $H^{2}$, we see that $\widetilde{\mathrm{SL}}_{2}$ is naturally a line bundle over $\boldsymbol{H}^{2}$. Topologically, such a bundle must be trivial, but, in this case, it is non-trivial in a metric sense. To be precise, $\widetilde{S L}_{2}$ is not isometric to $H^{2} \times \mathbb{R}$. This will be proved in $\$ 5$.

The isometry group of $\widetilde{\mathrm{SL}_{2}}$ preserves this bundle structure and is 4-dimensional as for $H^{2} \times \mathbb{R}$. However, Isom $\widetilde{\mathrm{SL}}_{2}$ has only two components, both orientation preserving which differs from the case of $H^{2} \times \mathbb{R}$. In particular, $\widetilde{\mathrm{SL}}_{2}$ admits no orientation reversing isometry. We will see later in this section, that if $M$ is a closed 3-manifold which admits a hyperbolic structure modelled on $\widetilde{\mathrm{SL}}_{2}$ then $M$ naturally inherits the structure of a Seifert bundle $\eta$ over an orbifold $X$, where $\chi(X)<0$ and $e(\eta) \neq 0$.

Now we must fill in some of the details of the preceding discussion. We will not describe the metric on the tangent bundle $T M$ of any Riemannian manifold $M$, but will be content to discuss the case when $M$ is the hyperbolic plane. First we introduce the concept of parallel translation of tangent vectors.

For the Euclidean plane $E^{2}$, it clearly makes sense to talk of a tangent vector at some point $x$ being parallel to a tangent vector at some other point $y$. But for other Riemannian 2-manifolds this cannot be done. What one can do is to consider a path $l$ on an oriented surface $M$ joining two points $x$ and $y$ and use $l$ to define the concept of parallel. This is best done when $l$ is a geodesic path. If $v$ is a vector tangent to $l$ at $x$, then the vector tangent to $l$ at $y$ with the same length as $v$ is called the parallel translate of $v$ by $l$. As the fibres at $x$ and $y$ are only 2-dimensional, one can clearly define the parallel translate by $l$ of any tangent vector at $x$ and one obtains an isometry of $T_{x} M$ with $T_{y} M$. If $l$ is not a geodesic, the definition is more complicated and we shall not discuss it. However, if $l$ is piecewise geodesic, parallel translation is easy to define. If $l$ is a loop, then parallel translation along $l$ induces a rotation of $T_{x} M$ called the holonomy of $l$ and the angle of rotation is called the holonomy angle. 
Clearly, the holonomy of any piecewise geodesic loop in $E^{2}$ is trivial, but for other surfaces this is not the case. We will give an example in the hyperbolic plane $H^{2}$. Let $l$ denote the loop consisting of the edges of a geodesic triangle $\Delta$ and let $x$ be a vertex of $\triangle$. It is easy to see, Fig. 4.14, that the holonomy angle of $l$ is $\pi-(\alpha+\beta+\gamma)$, where $\alpha, \beta$ and $\gamma$ are the angles of $\triangle$. By choosing $\triangle$ to be small, we can obtain arbitrarily small non-trivial holonomies.

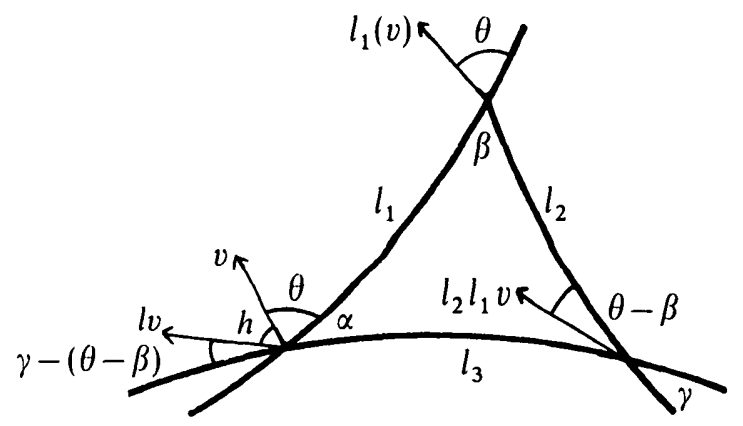

The loop $l=l_{3} l_{2} l_{1}$ has holonomy angle $h=\pi-(\alpha+\beta+\gamma)$

FIG. 4.14

Let $x$ be a point of $H^{2}$ and let $v$ be a point of $T H^{2}$ above $x$, that is, $v$ is a tangent vector at $x$. If $l$ is a geodesic of $H^{2}$ through $x$, there is a unique lift $\tau$ of $l$ to $T H^{2}$ such that $l$ passes through $v$ and for each point $y$ on $l, \pi(y)$ is the parallel translate by $l$ of $v$. We will say that a fibre of $T H^{2}$ is vertical and that a path $\widetilde{l}$ in $T H^{2}$ with the above properties is horizontal. The union of all such paths through $v$, one for each geodesic of $H^{2}$ through $x$, clearly forms a surface in $T H^{2}$. The tangent plane to this surface at $v$ will be called horizontal. We can define similarly the horizontal tangent plane at any other point of $T H^{2}$, giving a plane field on $T H^{2}$. It is natural to call a surface in $T H^{2}$ horizontal if its tangent plane at every point is horizontal. However, there are no horizontal surfaces in $T H^{2}$. Equivalently, the horizontal plane field defined above is not integrable. For suppose that $S$ is a horizontal surface in $T H^{2}$ and let $v$ be a point of $S$ above $x$ in $H^{2}$. The projection map $S \rightarrow H^{2}$ is a covering map. Hence, as $H^{2}$ is simply connected, $S$ meets each fibre of $T H^{2}$ exactly once. On the other hand, if $l$ is a loop based at $x$ with non-trivial holonomy, then the horizontal lift $I$ of $l$ which passes through $v$ meets the fibre above $x$ in two distinct points. As $\tau$ must lie in $S$, this is impossible. Note that as $H^{2}$ contains arbitrarily small loops with non-trivial holonomy, the horizontal plane field on $T^{2} H^{2}$ is not even locally integrable.

We will use this horizontal plane field on $T H^{2}$ to define the required metric on $T H^{2}$. Let $w$ be a point of $T H^{2}$ above $x$ in $H^{2}$. The tangent space $T_{w}\left(T H^{2}\right)$ to $T H^{2}$ at $w$ is the direct sum of two 2-dimensional subspaces $V$ and $H$, where $V$ consists of the tangents to $T_{x}$, the fibre of $T H^{2}$ above $x$, and $H$ is the horizontal plane at $w$. The inner product on $T_{w}\left(T H^{2}\right)$ which is needed to define the Riemannian metric on $T H^{2}$ is to be chosen so that $V$ and $H$ are orthogonal. Hence the terminology of horizontal and vertical. As $V$ is a tangent plane to $T_{x}$, which is a linear space with an inner product of its own, there is a natural inner product on $V$, which is essentially the same as that on $T_{x}$. Thus the induced Riemannian metric on $T_{x}$ will make $T_{x}$ isometric to $E^{2}$. The inner product on $H$ is chosen to be essentially the same as that on $T_{x}$ also. Precisely, let $p: T H^{2} \rightarrow H^{2}$ be the natural projection. Then $d p_{w}: T_{w}\left(T H^{2}\right) \rightarrow T_{x}$ sends $H$ to $T_{x}$ by a linear isomorphism and we ensure that this map is actually an isometry. 
If $f: H^{2} \rightarrow H^{2}$ is an isometry, then $d f: T H^{2} \rightarrow T H^{2}$ is also an isometry, when $T H^{2}$ is given the above metric. For clearly $d(d f)_{w}: T_{w}\left(T H^{2}\right) \rightarrow T_{d f(w)}\left(T H^{2}\right)$ sends the vertical and horizontal subspaces of $T_{w}\left(T H^{2}\right)$ to the vertical and horizontal subspaces of $T_{d f(w)}\left(T H^{2}\right)$ and preserves the inner product on each subspace. Let $U H^{2}$ be the submanifold of $T H^{2}$ consisting of tangent vectors of unit length. This is the unit tangent bundle of $H^{2}$. It is a bundle over $H^{2}$ with fibre $S^{1}$. Clearly $d f$ also gives an isometry of $U H^{2}$ to itself. Recall that $\mathrm{PSL}_{2} \mathbb{R}$ is the group of orientation preserving isometries of $H^{2}$. We know from $\S 1$ that $\mathrm{PSL}_{2} \mathbb{R}$ acts transitively on $U H^{2}$ and that the stabiliser of a point of $U H^{2}$ is trivial. It follows that we can identify $U H^{2}$ with $\mathrm{PSL}_{2} \mathbb{R}$. Now we have a metric on $\mathrm{PSL}_{2} \mathbb{R}$ and this in turn gives a metric on $\widetilde{S L}_{2}$, the universal covering of $U H^{2}$. We have just observed that $\mathrm{PSL}_{2} \mathbb{R}$ acts on $U H^{2}$ by isometries, when $U H^{2}$ has the metric we have described. Thus the induced metric on $\mathrm{PSL}_{2} \mathbb{R}$ is invariant under left multiplication. $\mathrm{PSL}_{2} \mathbb{R}$ acts on $H^{2}$ on the left in this article.) It follows that our metric on $\widetilde{\mathrm{SL}}_{2}$ is also invariant under left multiplication.

As $U H^{2}$ is a circle bundle over $H^{2}$, we see that $\widetilde{\mathrm{SL}}_{2}$ has the structure of a line bundle over $H^{2}$. Again we call the fibres of this bundle vertical. The horizontal plane field on $U H^{2}$ gives a plane field on $\widetilde{\mathrm{SL}}{ }_{2}$ which we again call horizontal. As the projection map $\widetilde{\mathrm{SL}}_{2} \rightarrow U H^{2}$ is a local isometry, this plane field is non-integrable. This shows directly that $\widetilde{\mathrm{SL}}_{2}$ is not isometric to $H^{2} \times \mathbb{R}$ by any isomorphism preserving fibres.

As $\pi_{1}\left(U H^{2}\right)$ is infinite cyclic, we have the exact sequence

$$
0 \longrightarrow \mathbb{Z} \longrightarrow \widetilde{\mathrm{SL}}_{2} \longrightarrow \mathrm{PSL}_{2} \mathbb{P} \longrightarrow 1
$$

where the $\mathbb{Z}$-subgroup of $\widetilde{\mathrm{SL}}_{2}$ is central. As $\mathrm{PSL}_{2} \mathbb{R}$ is centreless, the $\mathbb{Z}$-subgroup is exactly the centre of $\widetilde{S L}_{2}$. This extension does not split and the geometry of $H^{2}$ gives a very clear picture of the reason. Let $S$ denote the group of rotations of $H^{2}$ fixing some point $x$. Thus the circle $S$ acts by isometries on $U H^{2}$ also. Clearly, it acts on the circle of unit tangents at $x$ by rotating this circle. Now regard $S$ as a path in $\mathrm{PSL}_{2} \mathbb{R}$ starting at the identity, and lift this (uniquely) to a path in $\widetilde{\mathrm{SL}}_{2}$ starting at the identity. Each isometry of $\widetilde{\mathrm{SL}}_{2}$ preserves the line-bundle structure and the final isometry covers the identity map of $H^{2}$. The line above $x$ is left invariant by each isometry and is translated steadily in one direction. Hence the final isometry of $\widetilde{\mathrm{SL}}_{2}$ is not the identity. This shows that the pre-image in $\widetilde{\mathrm{SL}}_{2}$ of $S$ is a subgroup isomorphic to $\mathbb{R}$.

Now we can complete our discussion of the isometry group of $\widetilde{\mathrm{SL}}_{2}$. We already know a 3-dimensional isometry group of $\widetilde{\mathrm{SL}}_{2}$-namely $\widetilde{\mathrm{SL}}_{2}$ itself. The centre of $\widetilde{\mathrm{SL}}_{2}$ (which is infinite cyclic) acts on $\widetilde{\mathrm{SL}}_{2}$ preserving the line bundle structure over $H^{2}$ and covering the identity map of $H^{2}$. Each central element simply translates the fibres a fixed distance. This action of $\mathbb{Z}$ on $\widetilde{\mathrm{SL}}{ }_{2}$ extends to an action of $\mathbb{P}$ which again simply translates the fibres of $\widetilde{\mathrm{SL}}_{2}$. This action of $\mathbb{R}$ arises from an action of $S^{1}$ on $T H^{2}$ which preserves the bundle structure, covers the identity map of $H^{2}$ and rotates each fibre through the same angle. The definition of the metric on $T H^{2}$ shows that this action of $S^{1}$ is by isometries.

Clearly this action of $\mathbb{R}$ on $\widetilde{\mathrm{SL}}_{2}$ commutes with the action of $\widetilde{\mathrm{SL}}_{2}$ and intersects $\widetilde{\mathrm{SL}}_{2}$ precisely in the centre of $\widetilde{\mathrm{SL}}_{2}$. Hence the group $\Gamma$ of isometries of $\widetilde{\mathrm{SL}}_{2}$ generated by $\mathbb{R}$ and $\widetilde{S L}_{2}$ is a 4-dimensional Lie group and we have the exact sequence

$$
0 \longrightarrow \mathbb{R} \longrightarrow \Gamma \longrightarrow \mathrm{PSL}_{2} \mathbb{R} \longrightarrow 1 \text {. }
$$


Further $\Gamma$ acts on $\widetilde{\mathrm{SL}}_{2}$ preserving the bundle structure and the projection to $\mathrm{PSL}_{2} \mathbb{R}$ gives the induced action on the base space $H^{2}$ of this bundle. I now claim that $\Gamma$ is the identity component of Isom $\widetilde{S L}_{2}$. Results in $\$ 5$ show that the identity component of the stabiliser of a point of $\widetilde{\mathrm{SL}}_{2}$ under the action of Isom $\widetilde{\mathrm{SL}}_{2}$ can only be $\mathrm{SO}(3), \mathrm{SO}(2)$ or trivial. Thus Isom $\widetilde{\mathrm{SL}}_{2}$ must have dimension 3,4 or 6 . As $\Gamma$ is a 4-dimensional subgroup of Isom $\widetilde{S L}_{2}$, either it is the identity component of Isom $\widetilde{\mathrm{SL}}{ }_{2}$ or Isom $\widetilde{\mathrm{SL}}_{2}$ is 6-dimensional. In this last case $\widetilde{\mathrm{SL}}_{2}$ would be of constant curvature and so isometric to $S^{3}, E^{3}$ or $H^{3}$. We will show that this is impossible in $\S 5$.

Having described the identity component $\Gamma$ of Isom $\widetilde{S L}_{2}$, it remains to show that Isom $\widetilde{\mathrm{SL}}_{2}$ has only two components as stated earlier. Clearly, there are at least two components one of which is $\Gamma$ and the other, say $\Gamma^{\prime}$, contains isometries induced from orientation reversing isometries of $H^{2}$. Note that these isometries reverse the orientations of the fibres of $\widetilde{\mathrm{SL}}_{2}$ also and so are orientation preserving isometries of $\widetilde{\mathrm{SL}}_{2}$.

As $\Gamma$ acts transitively on $\widetilde{\mathrm{SL}}_{2}$, it suffices to show that the stabiliser of a point $w$ of $\widetilde{\mathrm{SL}}_{2}$ has only two components. Now the identity component of the stabiliser of $w$ is isomorphic to $S^{1}$ and leaves fixed the fibre of $\widetilde{\mathrm{SL}}_{2}$ through $w$. As this is a normal subgroup of the full stabiliser of $w$, it follows that any isometry $\alpha$ of $\widetilde{S L}_{2}$ fixing $w$ must leave invariant the fibre through $w$. We can compose $\alpha$ with an isometry in $\Gamma$ or $\Gamma^{\prime}$ to obtain an isometry $\beta$ which fixes $w$, leaves invariant the fibre through $w$ and whose differential at $w$ is the identity on the horizontal tangent plane at $w$. Let $x$ be the point of $H^{2}$ beneath $w$ and let $l$ be a piecewise geodesic loop in $H^{2}$ based at $x$ with non-trivial holonomy. Let $T$ be the horizontal lift of $l$ to $\widetilde{\mathrm{SL}}_{2}$ starting at $w$, and let $w_{1}$ denote the other end of $\tilde{l}$. Now $\beta(\bar{l})$ is a horizontal path in $\widetilde{\mathrm{SL}}_{2}$ which starts at $w$ and is tangent to $T$ there. Hence $\beta(\bar{l})$ equals $T$ and in particular $\beta$ must fix $w_{1}$. As $w$ and $w_{1}$ are distinct, it follows that $\beta$ fixes each point of the fibre of $\widetilde{\mathrm{SL}}_{2}$ through $w$ and hence $\beta$ is the identity. Hence the isometry $\alpha$ lies in $\Gamma$ or $\Gamma^{\prime}$, and it follows that Isom $\widetilde{\mathrm{SL}}_{2}$ has only two components as claimed. We have used the fact that if $\beta$ is an isometry of a complete Riemannian manifold $M$ which fixes a point $w$ and has differential at $w$ which is the identity, then $\beta$ must be the identity. This is because each geodesic through $w$ must be left fixed by $\beta$ and any point of $M$ lies on one of these geodesics.

We will show next that every closed 3-manifold with a geometric structure modelled on $\widetilde{\mathrm{SL}}_{2}$ is a Seifert fibre space, where the fibres of the Seifert fibration are the images of the fibres of $\widetilde{\mathrm{SL}}_{2}$. These fibres form a foliation of $\widetilde{\mathrm{SL}}_{2}$, and we have very much the same result as for $H^{2} \times \mathbb{R}$. Note that any manifold modelled on $\widetilde{\mathrm{SL}}_{2}$ inherits a foliation by lines or circles as Isom $\widetilde{\mathrm{SL}}_{2}$ leaves this foliation invariant.

THEOREM 4.15. Let $G$ be a discrete group of isometries of $\widetilde{\mathrm{SL}}_{2}$ acting freely. and with quotient $M$. The foliation of $\widetilde{\mathrm{SL}}_{2}$ by vertical lines descends to a foliation of $M$ and one of the following cases occurs.

(i) The foliation gives $M$ the structure of a line bundle over a non-closed surface.

(ii) The foliation of $M$ is a Seifert fibration.

(iii) The foliation of $M$ is by lines whose image in $M$ is not closed. In this case, $G$ must be isomorphic to $\mathbb{Z}, \mathbb{Z} \times \mathbb{Z}$ or the Klein bottle group.

Remark. Unlike the situation for the product geometries $E^{3}, S^{2} \times \mathbb{R}$ and $H^{2} \times \mathbb{R}$, the manifold $M$ does not also have the structure of a surface bundle over a 1-dimensional orbifold. 
Proof. The proof starts on exactly the same lines as the proof of Theorem 4.13. We have the exact sequence

$$
0 \longrightarrow \mathbb{R} \longrightarrow \operatorname{Isom}\left(\widetilde{\mathrm{SL}}_{2}\right) \stackrel{p}{\longrightarrow} \text { Isom } H^{2} \longrightarrow 1 \text {. }
$$

Hence if $K$ denotes $G \cap \mathbb{R}$ and $\Gamma$ denotes $p(G)$, we have the exact sequence<smiles>O[Y]C[I-][AlH]</smiles>

As $G$ is discrete, $K$ is infinite cyclic or trivial. If $K$ is infinite cyclic, we have case (ii) of the theorem. If $K$ is trivial and $\Gamma$ is discrete, we have case (i) of the theorem. Note that the base of the line bundle cannot be a closed surface. In fact, Isom $\widetilde{S L}_{2}$ does not contain the fundamental group of any closed hyperbolic surface. For if $G$ (and $\Gamma$ ) were isomorphic to $\pi_{1}(F)$ where $F$ is a closed hyperbolic surface, then $G$ is a subgroup of $p^{-1}(\Gamma)$, where

$$
0 \longrightarrow \mathbb{B} \longrightarrow p^{-1}(\Gamma) \longrightarrow \Gamma \longrightarrow 1
$$

Now $p^{-1}(\Gamma)$ contains $p^{-1}(\Gamma) \cap \widetilde{S L}_{2}$ which we denote by $G_{1}$ and we have

$$
0 \longrightarrow \mathbb{Z} \longrightarrow G_{1} \longrightarrow \Gamma \longrightarrow 1
$$

But our earlier discussion shows that $G_{1}$ is the fundamental group of the unit tangent bundle of $F$ and in particular the extension defining $G_{1}$ does not split. It follows that the extension describing $p^{-1}(\Gamma)$ also does not split, which contradicts our assumption.

We are left with the case when $K$ (which is $G \cap \mathbb{R}$ ) is trivial and $\Gamma=p(G)$ is indiscrete. Clearly, this yields a foliation of $M$ as in case (iii) and it remains to show that $G$ must be one of the three stated groups. It suffices, as before, to show that $G$ has an abelian subgroup of finite index. In the proof of Theorem 4.13, a crucial part in the argument was played by the projection $\operatorname{Isom}\left(H^{2} \times \mathbb{R}\right) \rightarrow \operatorname{Isom}(\mathbb{R})$. There is no projection Isom $\widetilde{\mathrm{SL}}_{2} \rightarrow$ Isom $\mathbb{R}$ and so a more sophisticated argument is needed. The argument needed is given in Thurston [66].

If $M$ is a closed 3-manifold with a geometric structure modelled on $\widetilde{\mathrm{SL}}_{2}$, then Theorem 4.15 shows that $M$ must inherit a Seifert fibration $\eta$ from the foliation of $\widetilde{\mathrm{SL}}_{2}$ by lines. Further, the base orbifold $X$ of this fibration is a quotient of $H^{2}$ by some isometry group and so $\chi(X)<0$. Finally we must have $e(\eta) \neq 0$. For if $e(\eta)$ were zero, $M$ would be finitely covered by $F \times S^{1}$ for some closed hyperbolic surface $F$. But we showed in the proof of Theorem 4.15 that $\pi_{1}(M)$ cannot contain $\pi_{1}(F)$ as a subgroup, because Isom $\widetilde{\mathrm{SL}}_{2}$ cannot contain such a subgroup.

To end this section, I want to discuss the unit tangent bundle of a closed orientable hyperbolic orbifold $X$. Recall the exact sequence

$$
0 \longrightarrow \mathbb{Z} \longrightarrow \widetilde{\mathrm{SL}}_{2} \stackrel{p}{\longrightarrow} \text { Isom } H^{2} \longrightarrow 1
$$

Choose an embedding of $\pi_{1}(X)$ in Isom $H^{2}$ and let $\Gamma=p^{-1}\left(\pi_{1}(X)\right)$. As $\Gamma$ acts freely on $\widetilde{\mathrm{SL}}_{2}$ as a group of isometries, we see that $\Gamma$ is the fundamental group of a Seifert 
fibre space $M$ which is the total space of a Seifert bundle $\eta$ over $X$. This is the unit tangent bundle of $X$. Let $k$ denote a generator of the centre of $\widetilde{\mathrm{SL}_{2}}$, and let $\bar{x}_{i}$ be a rotation through $2 \pi / \alpha_{i}$ in $\pi_{1}(X)$. Then there is $x_{i}$ in $\Gamma$ such that $x_{i}^{\alpha_{i}} \cdot k=1$, and $p\left(x_{i}\right)=\bar{x}_{i}$. This follows from our earlier discussion of the above extension and why it fails to split. Thus the Seifert invariants of $\eta$ are all $\left(\alpha_{i}, 1\right)$. It remains to calculate $e(\eta)$. If $X$ were actually a closed surface, we know that the total space of $\eta$ would be the unit tangent bundle of $X$. Thus we would have $e(\eta)=\chi(X)$. Now the naturality properties of $e(\eta)$ and $\chi(X)$ under finite coverings show that we must have $e(\eta)=\chi(X)$ for any closed orientable orbifold $X$. The same analysis applies if $X$ is non-orientable, so long as $X$ has no reflector curves.

The geometry of Nil. Nil is the 3-dimensional Lie group which consists of all $3 \times 3$ real upper triangular matrices of the form $\left(\begin{array}{lll}1 & x & z \\ 0 & 1 & y \\ 0 & 0 & 1\end{array}\right)$ under multiplication. It is also called the Heisenberg group. This group is nilpotent. In fact, one has the exact sequence

$$
0 \longrightarrow \mathbb{R} \longrightarrow \mathrm{Nil} \stackrel{p}{\longrightarrow} \mathbb{R}^{2} \longrightarrow 0,
$$

where the central $\mathbb{R}$ consists of those elements of Nil with $x=y=0$. Thus Nil naturally possesses the structure of a line bundle over $\mathbb{R}^{2}$. Note that this extension

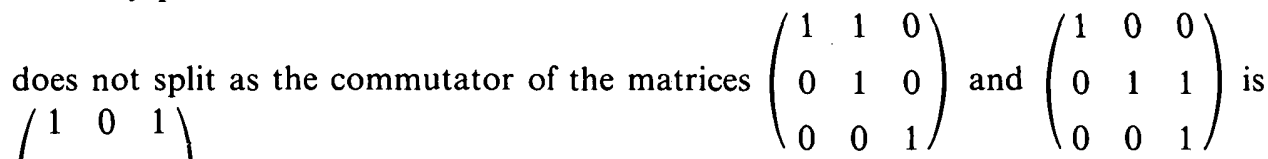
$\left(\begin{array}{lll}1 & 0 & 1 \\ 0 & 1 & 0 \\ 0 & 0 & 1\end{array}\right)$. As $\mathrm{Nil}$ is a Lie group, it admits a metric invariant under left multiplication, which I will describe shortly. With this metric, Nil is a line bundle over the Euclidean plane $E^{2}$, in the same way that $\widetilde{S L}_{2}$ is a line bundle over $H^{2}$. The fibres of Nil are called vertical, and the orthogonal plane field is called horizontal. If $u$ is a point of Nil and $p(u)$ is $x$ in the base space $E^{2}$ of Nil, and if $H_{u}$ denotes the horizontal tangent plane at $u$, then $d p_{u} \mid: H_{u} \rightarrow T_{x}$ preserves the inner product. However, Nil cannot be thought of as the universal covering of the unit tangent bundle of $E^{2}$. In fact, as all loops on $E^{2}$ have trivial holonomy, the unit tangent bundle of $E^{2}$ is isometric to $E^{2} \times S^{1}$ and so its universal covering is isometric to $E^{3}$. We will show in $\$ 5$, that $\mathrm{Nil}$ is not isometric to $E^{3}$. One can also show directly that the horizontal plane field on Nil is not integrable, by considering horizontal paths in Nil.

The isometry group of Nil can be described as follows and has a somewhat similar structure to Isom $\left(\widetilde{S L}_{2}\right)$. All isometries of Nil preserve orientation. Also all isometries of Nil preserve the bundle structure and hence induce an isometry of the base space $E^{2}$. There is an exact sequence

$$
0 \longrightarrow \mathbb{R} \longrightarrow \operatorname{Isom}(\mathrm{Nil}) \longrightarrow \operatorname{Isom}\left(E^{2}\right) \longrightarrow 1 \text {. }
$$

In particular, Isom (Nil) has exactly two components.

A nice example of a manifold with a geometric structure modelled on Nil is obtained by taking the quotient of Nil by the subgroup $\Gamma$ of $N$ consisting of all matrices in $N$ with integer entries. This manifold is a circle bundle $\eta$ over the torus with orientable total space and $e(\eta)=1$. 
Now I will give a few more details. First we need to describe the metric on Nil. In this case, the simplest and clearest way to do this seems to be to actually give a formula for the metric. We identify Nil with $\mathbb{R}^{3}$ so that $(x, y, z)$ in $\mathbb{R}^{3}$ corresponds to the matrix $\left(\begin{array}{lll}1 & x & z \\ 0 & 1 & y \\ 0 & 0 & 1\end{array}\right)$. Thus $\mathbb{R}^{3}$ now has the multiplication

$$
(x, y, z) \cdot\left(x^{\prime}, y^{\prime}, z^{\prime}\right)=\left(x+x^{\prime}, y+y^{\prime}, z+z^{\prime}+x y^{\prime}\right) .
$$

A Riemannian metric on $\mathbb{R}^{3}$ can be given by a formula for $d s^{2}$. It is easy to write down such a formula which is invariant under left multiplication for Nil. One chooses $d s^{2}$ arbitrarily at some point, and $d s^{2}$ is then determined at all other points by the group action. I shall choose $d s^{2}=d x^{2}+d y^{2}+d z^{2}$ at the origin. The corresponding invariant metric on $\mathbb{R}^{3}$ is given by

$$
d s^{2}=d x^{2}+d y^{2}+(d z-x d y)^{2} .
$$

So far we have only exhibited a 3-dimensional isometry group of Nil-namely Nil itself. Now there is an action of $S^{1}$ on Nil which is a group of automorphisms of Nil and this action preserves the above metric. The action preserves the bundle structure of Nil and induces a rotation on $E^{2}$ fixing the origin. If we identify $S^{1}$ with the interval $[0,2 \pi]$ with the ends identified and if we write $c=\cos \theta, s=\sin \theta$, then the point $\theta$ of $S^{1}$ acts on Nil by

$$
(\mathbf{x}, z) \longrightarrow\left(\rho_{\theta}(\mathbf{x}), z+\frac{1}{2} s\left(c y^{2}-c x^{2}-2 s x y\right)\right),
$$

where $\mathbf{x}$ denotes the vector $(x, y)$ and $p_{\theta}(\mathbf{x})$ denotes the effect of a rotation through $\theta$ on $\mathbf{x}$.

This somewhat mysterious looking formula can be rendered more reasonable by changing our identification of Nil with $\mathbb{R}^{3}$. This makes the induced multiplication on $\mathbb{R}^{3}$ look more mysterious while simplifying the action of $S^{1}$ on $\mathbb{R}^{3}$. This can be done in various ways. Here is one. We can identify Nil with the subset $G$ of $\mathbb{C}^{2}$ defined as $\left\{(u, v) \in \mathbb{C}^{2}: \operatorname{Im} v=|u|^{2}\right\}$ and with multiplication on $G$ defined by

$$
(u, v) \cdot\left(u^{\prime}, v^{\prime}\right)=\left(u+u^{\prime}, v+v^{\prime}+2 i u \bar{u}^{\prime}\right) .
$$

An isomorphism from Nil to $G$ can be given by the formula

$$
(x, y, z) \longrightarrow\left(\frac{1}{2}(x+i y), z-\frac{1}{2} x y+\frac{1}{4} i\left(x^{2}+y^{2}\right)\right) \text {. }
$$

The $S^{1}$-action on $G$ corresponding to the action on Nil is described simply by the formula $(u, v) \rightarrow\left(e^{i \theta} u, v\right)$.

The isometry group of Nil generated by Nil and this circle action is 4-dimensional. It follows, as when we discussed $\widetilde{\mathrm{SL}}_{2}$, that this group is the identity components of Isom Nil, we argue as for $\widetilde{S L}_{2}$. We need to find a loop $l$ in $E^{2}$ with a $(x, y, z) \rightarrow(x,-y,-z)$ is an isometry of Nil. In order to show that there are no more components of Isom Nil, we argue as for $\widetilde{S L}_{2}$. We need to find a loop $l$ in $E^{2}$ with a horizontal lift into Nil which is not a loop, i.e. the endpoints are distinct. Recall that the metric on $\mathbb{R}^{3}$ is given by $d s^{2}=d x^{2}+d y^{2}+(d z-x d y)^{2}$. It follows that the 
horizontal tangent plane at $(x, y, z)$ contains the vectors $(1,0,0)$ and $(0,1, x)$. Now it is clear that if $l$ is the boundary of the square in $E^{2}$ with vertices $(0,0),(0,1),(1,0)$ and $(1,1)$, then a horizontal lift of $l$ into Nil has distinct endpoints.

To end this section, we state without proof the result which describes 3-manifolds with a geometric structure modelled on Nil. The statement and proof are similar to those of Theorem 4.15 .

THEOREM 4.16. Let $G$ be a discrete group of isometries of Nil acting freely and with quotient $M$. The foliation of Nil by vertical lines descends to a foliation of $M$ and one of the following occurs.

(i) The foliation gives $M$ the structure of a line bundle over a non-closed surface.

(ii) The foliation of $M$ is a Seifert fibration.

(iii) The foliation of $M$ is by lines whose image in $M$ is not closed. In this case, $G$ must be isomorphic to $\mathbb{Z}, \mathbb{Z} \times \mathbb{Z}$ or the Klein bottle group.

If $M$ is a closed 3-manifold with a geometric structure modelled on Nil, then Theorem 4.16 shows that $M$ must inherit the structure of a Seifert bundle $\eta$ from the foliation of Nil by vertical lines. Further, the base orbifold $X$ of $\eta$ is a quotient of $E^{2}$ by some isometry group and so $\chi(X)=0$. Finally, we must have $e(\eta) \neq 0$. For if $e(\eta)$ were zero, then $M$ would be finitely covered by the 3-torus, and the 3-torus would also inherit a natural Seifert fibration from Nil. Thus we would have a discrete group $G$ of isometries of Nil, with $G$ free abelian of rank 3, and the projection Isom (Nil) $\rightarrow \operatorname{Isom}\left(E^{2}\right)$ restricted to $G$ has kernel $\mathbb{Z}$ with image $\mathbb{Z} \times \mathbb{Z}$. As $G$ is abelian, this extension should split. However, it cannot split, as the commutator of any two elements of Nil which project to independent translations of $E^{2}$ is always non-trivial.

If $M$ is a closed 3-manifold modelled on Nil, it has a natural Seifert fibration. The orthogonal plane field to this foliation of $M$ by circles is not integrable, so that $M$ cannot be given the structure of a surface bundle over a 1-dimensional orbifold with the surface fibres orthogonal to the fibres of the Seifert bundle. The same was true for manifolds modelled on $\widetilde{S L}_{2}$. However, some closed manifolds modelled on Nil can be given the structure of a surface bundle over a 1-dimensional orbifold. The surface fibres are each a union of fibres of the Seifert bundle. This structure can be obtained as follows.

Let $M$ be a closed 3-manifold modelled on Nil. Recall that Nil is a line bundle over $E^{2}$ and that $\pi_{1}(M)$ acts on Nil preserving this structure. Thus $M$ inherits the structure of a Seifert bundle $\eta$ over an orbifold $X$, which is the quotient of $E^{2}$ by some group of isometries. Let $l$ be a geodesic of $E^{2}$. Then $E^{2}$ has a foliation by lines parallel to $l$, and Nil has a corresponding foliation by planes each of which lies above one of these lines in $E^{2}$. Suppose that some element of $\pi_{1}(X)$ translates along $l$, and that the foliation of $E^{2}$ by lines parallel to $l$ is preserved by $\pi_{1}(X)$. Then the corresponding foliation of Nil by planes is preserved by $\pi_{1}(M)$, and each leaf has compact image in $M$. Hence $M$ has the structure of a surface bundle over a 1-dimensional orbifold where the fibres are tori or Klein bottles. As $M$ is orientable, a Klein bottle cannot occur as a regular fibre.

We will consider the case where the base orbifold is a circle. Thus $M$ is a bundle over $S^{1}$ with fibre the torus. Also $\pi_{1}(X)$ must be isomorphic to $\mathbb{Z} \times \mathbb{Z}$. The glueing map of this bundle can be understood in a similar way to that we used for the 
product geometries. One has a flow on Nil whose orbits are the horizontal curves orthogonal to the planes of our foliation of Nil, and this flow descends to a flow on $M$ which preserves the structure of a torus bundle over $S^{1}$. If $t$ is the smallest positive number such that each torus fibre is invariant, then the induced diffeomorphism $\phi_{t}$ of a fibre is the glueing map. As the flow on Nil preserves vertical lines, $\phi_{t}$ must preserve the vertical circles of the fibre torus. Hence the matrix in $\mathrm{SL}_{2}(\mathbb{Z})$ which describes the action of $\phi_{t}$ on the first homology group of the fibre must be of the form $\left(\begin{array}{ll}1 & n \\ 0 & 1\end{array}\right)$, when we take the first basis element to be represented by a vertical circle. One cannot have $n=0$, and the clearest way to see this is to look at the structure of $\pi_{1}(M)$ and identify $n$ with the Euler number of the Seifert bundle $\eta$.

We know that $M$ has the structure of a circle bundle over the torus with orientable total space and non-zero Euler number $e$. Thus $\pi_{1}(M)$ can be presented

$$
\left\{k, a, b:[k, a]=1,[k, b]=1,[a, b]=k^{e}\right\} .
$$

When we express $M$ as a torus bundle over $S^{1}$ as above, the fundamental group of the fibre torus is the subgroup of $\pi_{1}(M)$ generated by $k$ and $a$. The action of the glueing map is described by calculating how $b$ acts by conjugation on this subgroup of $\pi_{1}(M)$. As $b$ commutes with $k$ and $a^{-1} b^{-1} a b=k^{e}$, we see that $b^{-1} a b=a k^{e}$, so that the required matrix is $\left(\begin{array}{ll}1 & e \\ 0 & 1\end{array}\right)$.

The geometry of Sol. This geometry has the least symmetry of all the eight geometries as the identity component of the stabiliser of a point is trivial. The Lie group Sol is most easily defined as a split extension of $\mathbb{R}^{2}$ by $\mathbb{R}$. Thus one has the exact sequence

$$
0 \longrightarrow \mathbb{R}^{2} \longrightarrow \text { Sol } \longrightarrow \mathbb{R} \longrightarrow 0,
$$

and $t$ in $\mathbb{R}$ acts on $\mathbb{R}^{2}$ by the map sending $(x, y)$ to $\left(e^{t} x, e^{-t} y\right)$. For a given non-zero $t$, this is a linear isomorphism of $\mathbb{R}^{2}$ with determinant one and distinct real eigenvalues. Such a linear map is often called a hyperbolic isomorphism of $\mathbb{R}^{2}$. It should not be surprising that Sol is the correct geometry for those torus bundles over $S^{1}$ with hyperbolic glueing map. A homeomorphism of the torus $T$ determines an automorphism of $H_{1}(T) \cong \mathbb{Z} \oplus \mathbb{Z}$ and this is of hyperbolic type, if no eigenvalue has absolute value 1 . In this dimension, this condition implies, in particular, that the eigenvalues are real.

If we identify Sol with $\mathbb{R}^{3}$ so that the $x y$-plane corresponds to the normal subgroup $\mathbb{R}^{2}$, we can easily write down the multiplication of Sol, and an invariant metric. The multiplication is given by

$$
(x, y, z)\left(x^{\prime}, y^{\prime}, z^{\prime}\right)=\left(x+e^{-z} x^{\prime}, y+e^{z} y^{\prime}, z+z^{\prime}\right) .
$$

Clearly $(0,0,0)$ is the identity and the $x y$-plane is a normal subgroup isomorphic to $\mathbb{R}^{2}$, with the correct conjugation action of Sol on it. A left-invariant metric on $\mathbb{R}^{3}$ is given by the formula $d s^{2}=e^{2 z} d x^{2}+e^{-2 z} d y^{2}+d z^{2}$.

We will show in $\$ 5$ that the full isometry group of Sol has eight components and that the identity component is Sol acting on itself by left multiplication. The 
stabiliser of the origin is isomorphic to $D(4)$, the dihedral group of order eight, and consists of the linear maps of $\mathbb{R}^{3}$ given by $(x, y, z) \rightarrow( \pm x, \pm y, z)$ and $(x, y, z) \rightarrow( \pm y, \pm x,-z)$. This group will be denoted by $\Sigma$. One can see at once that these eight maps really are isometries of Sol, and are actually isomorphisms of Sol.

The surfaces $z=$ constant form a 2-dimensional foliation of Sol which is preserved by all the isometries of Sol. The induced metric on the plane $z=$ constant of $\mathbb{R}^{3}$ makes this plane isometric to $E^{2}$. Thus any 3-manifold $M$ with a geometric structure modelled on Sol inherits a natural 2-dimensional foliation and the leaves must be planes, annuli, Moebius bands, tori or Klein bottles. It is a surprising fact that this foliation always has leaves which are closed subsets of $M$.

THEOREM 4.17. Let $G$ be a discrete subgroup of Isom (Sol) which acts freely on Sol with quotient $M$. Then the natural 2-dimensional foliation of $M$ gives $M$ the structure of a bundle over a 1-dimensional orbifold.

Proof. Let $G_{0}$ denote the intersection of $G$ with the identity component of Isom (Sol). Thus $G_{0}$ has index at most eight in $G$. The exact sequence

$$
0 \longrightarrow \mathbb{R}^{2} \longrightarrow \text { Sol } \stackrel{p}{\longrightarrow} \mathbb{R} \longrightarrow 0
$$

yields the exact sequence

$$
0 \longrightarrow G_{0} \cap \mathbb{R}^{2} \longrightarrow G_{0} \longrightarrow p\left(G_{0}\right) \longrightarrow 0 \text {. }
$$

As $G$ is a discrete subgroup of Isom (Sol), $G_{0} \cap \mathbb{R}^{2}$ must be a discrete subgroup of $\mathbb{R}^{2}$. Hence $G_{0} \cap \mathbb{R}^{2}$ must be $1, \mathbb{Z}$ or $\mathbb{Z} \times \mathbb{Z}$.

If $G_{0} \cap \mathbb{R}^{2}$ is isomorphic to $\mathbb{Z} \times \mathbb{Z}$, the natural foliation of $M$ has compact leaves, and the result of Theorem 4.17 is clear.

Next we consider the case when $G_{0} \cap \mathbb{R}^{2}$ is isomorphic to $\mathbb{Z}$. In this case $G_{0}$ must equal $G_{0} \cap \mathbb{R}^{2}$, for the structure of Sol shows that no cyclic subgroup $C$ of $\mathbb{R}^{2}$ can have $g^{-1} C g=C$, where $g$ is in Sol, unless $g$ lies in $\mathbb{R}^{2}$. This is because the matrix $\left(\begin{array}{ll}e^{t} & 0 \\ 0 & e^{-t}\end{array}\right)$ has no eigenvalue equal to 1 or -1 . Hence Sol/ $G_{0}$ has the structure of a bundle over a 1-dimensional orbifold - the fibre is $S^{1} \times \mathbb{R}$ and the base is $\mathbb{R}$. As Sol/G is the quotient of Sol $/ G_{0}$ by the action of a finite group which must preserve the bundle structure, it follows that $\mathrm{Sol} / G$ is also a bundle over a 1-dimensional orbifold. Further, the base must be $\mathbb{R}$ or a half open interval with one reflexion point and each fibre must be an open annulus or Moebius band.

Finally, we consider the case when $G_{0} \cap \mathbb{R}^{2}$ is trivial. In this case, the projection $p:$ Sol $\rightarrow \mathbb{R}$ induces an isomorphism of $G_{0}$ with a subgroup of $\mathbb{R}$. Thus $G_{0}$ is a discrete abelian subgroup of Sol, with $G_{0} \cap \mathbb{R}^{2}$ trivial. We will see below that this forces $G_{0}$ to be cyclic. If we assume this, then Sol $/ G_{0}$ clearly has the structure of a bundle with fibre $\mathbb{R}^{2}$ over $\mathbb{R}$ or $S^{1}$. As Sol/G is the quotient of Sol/ $G_{0}$ by the action of a finite group which preserves the bundle structure, it follows that $\mathrm{Sol} / G$ has the structure of a bundle over a 1-dimensional orbifold. The fibres must all be $\mathbb{R}^{2}$ as this is the only surface finitely covered by $\mathbb{R}^{2}$ and so the base cannot have any reflexion points. Hence Sol/G is a bundle with fibre $\mathbb{R}^{2}$ and base which must be $\mathbb{R}$ or $S^{1}$. 
It remains to show that if $G_{0}$ is a discrete abelian subgroup of Sol and $G_{0} \cap \mathbb{R}^{2}$ is trivial, then $G_{0}$ is cyclic. Let $(x, y, z)$ be a non-trivial element of $G_{0}$. In particular $z$ is non-zero. Then $G_{0}$ is a subgroup of $C(x, y, z)$, the centraliser in Sol of $(x, y, z)$. Now a direct calculation, which we perform below, shows that $C(x, y, z)$ is isomorphic to $\mathbb{R}$. As $G_{0}$ is a discrete subgroup, it follows that $G_{0}$ is cyclic as required.

Now we demonstrate the claim that $C(x, y, z)$ is isomorphic to $\mathbb{R}$. The formula for multiplication of Sol given earlier shows that $\left(x^{\prime}, y^{\prime}, z^{\prime}\right)$ commutes with $(x, y, z)$ if and only if

and

$$
x+e^{-z} x^{\prime}=x^{\prime}+e^{-z^{\prime} x}
$$

$$
y+e^{-z} y^{\prime}=y^{\prime}+e^{-z^{\prime} y} .
$$

As $z$ is non-zero, $e^{-z}$ is not equal to 1 and so, given any value of $z^{\prime}$, there is a unique value for $x^{\prime}$ and for $y^{\prime}$ which satisfies these equations. Hence the homomorphism $p:$ Sol $\rightarrow \mathbb{R}$, which simply projects $(x, y, z)$ onto its $z$-coordinate restricts to a homomorphism $C(x, y, z) \rightarrow \mathbb{R}$ which is an isomorphism. This completes the proof of Theorem 4.17.

If $G$ is a discrete subgroup of Sol with $G \cap \mathbb{R}^{2} \cong \mathbb{Z} \times \mathbb{Z}$ and $p(G) \cong \mathbb{Z}$, generated by $t$, then Sol/G is naturally a torus bundle over $S^{1}$. Further, the glueing map of the bundle is a homeomorphism $T \rightarrow T$ which is covered by the linear map $\mathbb{B}^{2} \rightarrow \mathbb{R}^{2}$ with matrix $\left(\begin{array}{ll}t^{t} & 0 \\ 0 & e^{-t}\end{array}\right)$ with respect to some basis. Hence the glueing map is hyperbolic. Not every value of $t$ can arise in this way. For if we take a basis of $G \cap \mathbb{R}^{2}$ as basis of $\mathbb{R}^{2}$, the linear map $\mathbb{R}^{2} \rightarrow \mathbb{R}^{2}$ will be represented by an integral matrix. In particular, the eigenvalues, which are $e^{t}$ and $e^{-t}$, will be algebraic integers.

Conversely, suppose one is given a torus bundle over $S^{1}$ with orientation preserving hyperbolic glueing map and total space $M$. One can choose this glueing map so as to lift to a linear automorphism $\wedge$ of $\mathbb{R}^{2}$ and it will leave some lattice invariant precisely because it is a lift of a diffeomorphism of the torus. Suppose that the trace of $\wedge$ is positive. Then the two eigenvalues of $\wedge$ are also positive. As their product is 1 , we can write them as $e^{t}$ and $e^{-t}$, and there is a basis of $\mathbb{R}^{2}$ such that the matrix of $\wedge$ becomes $\left(\begin{array}{ll}e^{t} & 0 \\ 0 & e^{-t}\end{array}\right)$. Now it is clear that $M$ admits a geometric structure modelled on Sol and that $\pi_{1}(M)$ acts on Sol as a subgroup of Sol.

If one wants to put a Sol-structure on the total space of a torus bundle over $S^{1}$ whose glueing map is hyperbolic but may not be orientation preserving or may not have positive trace, then one will need to use a subgroup of Isom (Sol) which is not a subgroup of Sol. For example, let $t$ denote the element $(0,0, t)$ of Sol and let $g$ denote the isometry of Sol given by $g(x, y, z)=(-x,-y, z)$. Then conjugating the normal $\mathbb{R}^{2}$ in Sol by $g t$ gives a linear automorphism of $\mathbb{R}^{2}$ whose matrix is the product of $\left(\begin{array}{ll}e^{t} & 0 \\ 0 & e^{-t}\end{array}\right)$ and $\left(\begin{array}{rr}-1 & 0 \\ 0 & -1\end{array}\right)$. This product is $\left(\begin{array}{cc}-e^{t} & 0 \\ 0 & -e^{-t}\end{array}\right)$ and so we have an orientation preserving linear automorphism of $\mathbb{R}^{2}$ with negative trace. If we let $h$ denote the isometry of Sol given by $h(x, y, z)=(x,-y, z)$, then conjugation of $\mathbb{R}^{2}$ by $h t$ gives a linear automorphism of $\mathbb{R}^{2}$ with matrix $\left(\begin{array}{cc}e^{t} & 0 \\ 0 & -e^{-t}\end{array}\right)$, which is orientation reversing. One can now show easily that any torus bundle over $S^{1}$ with hyperbolic glueing map admits a Sol-structure. 


\section{\$5. The classification of the 3-dimensional geometries}

In this section I will sketch Thurston's proof that there are only eight 3-dimensional geometries in which we should be interested. Then I will show, as promised in $\$ 4$, that if a closed 3-manifold admits a geometric structure then the geometry involved is unique. First, we need to spend a little time discussing exactly what is to be classified and what is meant by the term 'geometry'.

In our context, there seem to be three distinct, but related, approaches to geometry which one can take. Often these are combined in various ways. The first approach is the classical one exemplified by Euclid in which one discusses only points, lines, incidence relations, angles and length. This approach can be used to study non-Euclidean geometries also. A second approach which emerged in the nineteenth century is that of differential geometry. Here the geometry of the Euclidean plane $E^{2}$ is recovered from the standard Riemannian metric on $E^{2}$ given by the formula $d s^{2}=d x^{2}+d y^{2}$, as discussed in $\S 1$. A third approach, formulated by Klein, is to say simply that if $X$ is a set and $G$ is a group acting on $X$, then the geometry of the pair $(X, G)$ is the study of those properties of $X$ left invariant by $G$. This approach is sometimes equivalent to the previous approaches.

For example, consider the plane $\mathbb{R}^{2}$, with its standard Riemannian metric and isometry group $I$. Then the pair $(X, G)$ which Klein would consider is the pair $\left(\mathbb{R}^{2}, I\right)$. To say that Klein's approach is equivalent to the differential geometric approach, means simply that given the pair $\left(\mathbb{R}^{2}, I\right)$ one can recover the standard Riemannian metric on $\mathbb{R}^{2}$. Now $I$ acts on $\mathbb{R}^{2}$ so that the stabiliser of any point is isomorphic to $\mathrm{O}(2)$. In particular, the stabiliser of any point is compact. It is a standard fact that this means that we can find a metric on $\mathbb{R}^{2}$ which is $I$-invariant. To find such a metric, choose a point $x$ in $\mathbb{R}^{2}$. As $I_{x}$, the stabiliser of $x$, is compact there is a quadratic form $q$ on $T_{x}$, the tangent plane at $x$, which is $I_{x}$-invariant. One can find $q$ by starting with a random quadratic form on $T_{x}$ and averaging it under the action of $I_{x}$. As $I$ acts transitively on $\mathbb{R}^{2}$, the action of $I$ on $\mathbb{R}^{2}$ determines uniquely the quadratic form to be assigned to $T_{y}$ for all $y$ in $\mathbb{R}^{2}$, in order to obtain a $I$-invariant Riemannian metric on $\mathbb{R}^{2}$. This metric is determined by the quadratic form $q$ on $T_{x}$ and, as $I_{x}$ is $\mathrm{O}(2), q$ must be a scalar multiple of the quadratic form $d x^{2}+d y^{2}$. Thus the $I$-invariant metric we obtain must be a scalar multiple of the standard Euclidean metric. Hence we have recovered the standard Euclidean plane $E^{2}$ from the pair $\left(\mathbb{R}^{2}, I\right)$.

The same arguments apply in general to show that if $X$ is a manifold and $G$ is a group which acts transitively on $X$ with compact point stabilisers, then there is a $G$-invariant metric on $X$ which is determined by the quadratic form on the tangent space of a single point of $X$. As $G$ acts transitively on $X$, this metric is automatically complete. If $X$ is $n$-dimensional and the stabiliser of a point of $X$ is $\mathrm{O}(n)$, then one will obtain a $G$-invariant metric on $X$ which is unique up to a scalar multiple as in the case of the pair $\left(\mathbb{R}^{2}, I\right)$ discussed above. However, in general, there are many $G$-invariant metrics on a manifold $X$ and they may give $X$ very different properties. An interesting and relevant example is to start with a Lie group $G$ and consider the pair $(G, G)$. Thus one is interested in $G$-invariant metrics on $G$. Milnor [38] has studied the possible curvatures of such metrics. He gives a complete discussion of the possibilities in the case of 3-dimensional Lie groups.

Recall from the introduction the definition that $M^{n}$ has a geometric structure if it admits a complete locally homogeneous metric. This means that the universal covering space $X$ of $M$ has a complete homogeneous metric, by a theorem of 
Singer [63], so that the isometry group of $X$ acts transitively. It is automatic that the stabiliser of a point of $X$ is compact. Thus we have obtained a geometry $(X, G)$ with $G$ being the isometry group of $X$.

Now we turn to the classification of the 3-dimensional geometries. By a geometry, we shall mean a pair $(X, G)$ where $X$ is a manifold and $G$ acts transitively on $X$ with compact point stabilisers. Two geometries $(X, G)$ and $\left(X^{\prime}, G^{\prime}\right)$ are equivalent if there is a diffeomorphism of $X$ with $X^{\prime}$ which throws the action of $G$ onto the action of $G^{\prime}$. In particular, $G$ and $G^{\prime}$ must be isomorphic. If $\tilde{X}$ denotes the universal covering of $X$, there is a natural geometry $(\tilde{X}, \tilde{G})$ where $\tilde{G}$ consists of all diffeomorphisms of $\tilde{X}$ which are lifts of elements of $G$. Hence it is reasonable to restrict our attention to geometries $(X, G)$ where $X$ is simply connected. We shall also restrict our attention to geometries where $G$ is maximal. Thus if $X$ is $\mathbb{R}^{2}$, we shall ignore the geometry $\left(\mathbb{R}^{2}, \mathbb{R}^{2}\right)$, where $\mathbb{R}^{2}$ acts on itself by translation, in favour of the geometry $\left(\mathbb{R}^{2}, I\right)$ discussed earlier. A final restriction which we need to impose in dimension three is that there exists a subgroup $H$ of $G$ which acts on $X$ as covering group and has compact quotient. We will say that the geometry admits a compact quotient.

THEOREM 5.1 (Thurston). Any maximal, simply connected, 3-dimensional geometry which admits a compact quotient is equivalent to one of the geometries $(X$, Isom $X)$ where $X$ is one of $E^{3}, H^{3}, S^{3}, S^{2} \times \mathbb{R}, H^{2} \times \mathbb{R}, \widetilde{\mathrm{SL}_{2} \mathbb{R}}$, Nil, or Sol, as discussed in $\$ 4$.

Remarks. The classification of 3-dimensional geometries which are not necessarily maximal and may not admit compact quotients is perfectly feasible. In fact, Kulkarni (unpublished) has carried out a finer classification in which one considers pairs $(G, H)$ where $G$ is a Lie group, $H$ is a compact subgroup and $G / H$ is a simply connected 3-manifold and the pairs $(G, H)$ and $\left(G^{\prime}, H^{\prime}\right)$ are equivalent if there is an isomorphism $G \rightarrow G^{\prime}$ sending $H$ to a conjugate of $H^{\prime}$. Thus, for example, the geometry $S^{3}$ arises from three distinct such pairs-namely $\left(S^{3}, e\right), \quad(U(2), S O(2))$ and (SO (4), SO (3)).

In relativity theory and cosmology, classifications of 3-dimensional geometries are important. See the book [82] by Ryan and Shepley. For example, the Bianchi classification consists of the classification of all simply connected 3-dimensional Lie groups up to isomorphism.

Finally, I should mention another geometric approach. Throughout this article I have considered only Riemannian manifolds, but one can also consider pseudoRiemannian manifolds in which the quadratic form attached to the tangent space at a point, need not be positive definite. A complete pseudo-Riemannian 3-manifold of constant curvature is the quotient of 3-dimensional Lorentz space by a group of isometries. These manifolds are not classified completely, but all the Seifert fibre spaces which admit a geometric structure modelled on $\widetilde{\mathrm{SL}}_{2}$ also admit a complete Lorentz metric of constant curvature. See Kulkarni's articles $[80,81]$ and the article of Kulkarni and Raymond [29].

Proof. I will not attempt to fill in all the details in the proof, but will present the main outline. Given a geometry $(X, G)$ as described, we first put a $G$-invariant metric on $X$. Next we choose $x$ in $X$ and consider the identity component $I\left(G_{x}\right)$ of the stabiliser $G_{x}$ of $x$. As $G$ acts on $X$ by isometries, $G_{x}$ acts on $T_{x}$, the tangent space at $x$, preserving the inner product given by our metric on $X$. Hence $G_{x}$ is naturally a 
subgroup of $\mathrm{O}(3)$. As $I\left(G_{x}\right)$ is connected, it must be trivial, $\mathrm{SO}(2)$ or $\mathrm{SO}(3)$. We consider these cases in reverse order.

If $I\left(G_{x}\right)$ is $\mathrm{SO}(3)$, then all sectional curvatures of $X$ at all points are the same. Thus $X$ has constant curvature and the geometry $(X, G)$ is equivalent to one of $S^{3}$, $E^{3}$ or $H^{3}$, depending on whether the sectional curvatures of $X$ are $>0,=0$ or $<0$. This is proved using the developing map for the geometric structure on $X$. See [66].

If $I\left(G_{x}\right)$ is $\mathrm{SO}(2)$, let $L_{x}$ be the 1-dimensional subspace of $T_{x}$ fixed by $I\left(G_{x}\right)$ and let $P_{x}$ be the orthogonal plane in $T_{x}$. As $I\left(G_{x}\right)$ is a normal subgroup of $G_{x}$, both $L_{x}$ and $P_{x}$ are invariant under the action of $G_{x}$ on $T_{x}$. The lines $L_{x}$ form a $G$-invariant line field on $X$ and the planes $P_{x}$ form an orthogonal $G$-invariant plane field. As $X$ is simply connected, we can choose coherent orientations on the $L_{x}$, so as to obtain a unit vector field $V_{x}$ on $X$. This vector field may not be $G$-invariant, as $G$ could reverse the direction of the vector field, but it will remain invariant under some subgroup $G_{1}$ of $G$ of index at most two. The vector field $V_{x}$ and the plane field $P_{x}$ are both $G_{1}$-invariant and so descend to any manifold covered by $X$ with covering group contained in $G_{1}$. The vector field $V_{x}$ also defines a flow $\phi_{t}$ on $X$ which clearly preserves the plane field $P_{x}$. Again this flow descends to manifolds covered by $X$ so long as the covering group is contained in $G_{1}$.

The plane $P_{x}$ inherits an inner product from that on $T_{x}$ and we will see that the flow $\phi_{t}$ on $X$ preserves this inner product. For let $w$ be a non-zero vector in $P_{x}$ and suppose that $\left\|d \phi_{t}(w)\right\|=A_{t}\|w\|$ where $\|\quad\|$ denotes the length of a tangent vector to $X$ determined by our Riemannian metric on $X$. Then the same equation holds for any vector in $P_{x}$ as the flow $\phi_{t}$ commutes with the action of $I\left(G_{x}\right)$ on $P_{x}$. (Recall that $I\left(G_{x}\right)$ is SO (2).) As the flow $\phi_{t}$ preserves the vector field $V_{x}$ on $X$, we see that the diffeomorphism $\phi_{t}$ multiplies the volume element $d V$ of $X$ by a constant factor $A_{t}$. Now we use the fact that $X$ admits a compact quotient $M$. By taking a double cover, if necessary, we can suppose that the covering group of $M$ lies in $G_{1}$ so that our flow and orthogonal plane field on $X$ both descend to $M$. Now $M$ has finite volume and the diffeomorphism $\phi_{t}$ of $M$ has the effect of multiplying this volume by $A_{t}$. Of course, it follows that $A_{t}$ must be 1 , for all $t$, as claimed.

There are now two cases according to whether or not the plane field $P_{x}$ is integrable. If it is integrable, one can show that up to scale changes $X$ must be isometric to $S^{2} \times \mathbb{R}, H^{2} \times \mathbb{R}$ or $E^{2} \times \mathbb{R}$. Of course, the case $E^{2} \times \mathbb{R}$ is not of interest as we only want maximal geometries. If $P_{x}$ is not integrable, one can show that up to scale changes $X$ must be isometric to $S^{3}, \widetilde{\mathrm{SL}_{2} \mathbb{R}}$ or Nil, and this time we omit the case $S^{3}$ as we only want maximal geometries. One can prove these statements by using the developing map for the geometric structure on $X$ again. For it is clear that the local picture in $X$ must be one of the six cases described.

Finally, we come to the case when $I\left(G_{x}\right)$ is trivial. This implies that the identity component $G_{e}$ of $G$ acts transitively and freely on $X$, so that $X$ is naturally identified with the Lie group $G_{e}$. Again we need to use the hypothesis that the geometry $(X, G)$ admits a compact quotient. As $G_{e}$ must be of finite index in $G$, it follows that $G_{e}$ has a discrete subgroup $H$ such that $G_{e} / H$ is compact.

A Lie group $G$ is called unimodular if its left invariant Haar measure is also right invariant. If $G$ has a discrete subgroup $H$ such that $H \backslash G$ has finite measure inherited from the left invariant Haar measure on $G$, then $G$ must be unimodular. This is because the action of $G$ on itself by right multiplication descends to an action of $G$ on $H \backslash G$ which must preserve the total measure of $H \backslash G$. Hence the group $G_{e}$ in which we are interested must be unimodular. 
In [38], Milnor considered $G$-invariant metrics on a Lie group $G$. If $G$ is 3-dimensional and unimodular, he shows that there are only six possible Lie algebras for $G$. Equivalently, there are only six simply connected, unimodular Lie groups. These groups are $S^{3}, \widetilde{\mathrm{SL}_{2} \mathbb{R}}$, Isom $E^{2}$, Sol, Nil and $\mathbb{R}^{3}$, where $\sim$ denotes universal cover. See p. 307 of [38]. Milnor writes $E(1,1)$ instead of Sol. This is the group of isometries of Minkowski 2-space.

Recall that we are considering a geometry $(X, G)$ such that $X=G_{e}$. If $X$ were $S^{3}$, this geometry would not be maximal, as it would be a subgeometry of $\left(S^{3}, \mathrm{O}(4)\right)$. If $X$ were $\widetilde{\mathrm{SL}_{2} \mathbb{R}}$, Nil or $\mathbb{R}^{3}$, similar remarks apply. If $X$ were $\widetilde{\operatorname{Isom} E^{2}}$, this geometry would not be maximal as Isom $E^{2}$ is a subgroup of Isom $E^{3}$ and so the geometry $(X, G)$ would be a subgeometry of $\left(E^{3}\right.$, Isom $\left.E^{3}\right)$. Hence the only possibility is that $X$ is the Lie group Sol.

We still need to show that for any left invariant metric on Sol, one must have $I\left(G_{x}\right)$ trivial, where $G$ is the full isometry group. One could use an indirect argument based on the classification of the previous seven geometries. For if Sol admits a left invariant metric such that $I\left(G_{x}\right)$ is non-trivial, then we must have a subgeometry of one of the previous seven geometries. However, one can show that for none of the seven geometries does the isometry group contain a subgroup isomorphic to Sol. This follows from Theorem 5.2. However, this argument would not help us decide what $G_{x}$ itself should be. A more direct argument is provided by Milnor. For he shows (see p. 307 of [38] again) that a left invariant metric on Sol determines in a canonical way, an orthonormal basis $e_{1}, e_{2}, e_{3}$ of the tangent space $T$ at the identity of Sol. These tangent vectors are the eigenvectors of a certain very natural selfadjoint linear map $L: T \rightarrow T$ and the eigenvalues $\lambda_{1}, \lambda_{2}$ and $\lambda_{3}$ must satisfy $\lambda_{1}>0$, $\lambda_{2}<0$ and $\lambda_{3}=0$, after reordering the vectors $e_{i}$ if necessary. Any isometry of Sol fixing the identity induces an orthogonal isomorphism $\theta$ of $T$ which must satisfy $\theta\left(e_{3}\right)= \pm e_{3}, \quad \theta\left(e_{1}\right)= \pm e_{1}$ or $\pm e_{2}$, and $\theta\left(e_{2}\right)= \pm e_{1}$ or $\pm e_{2}$. Further, if $\theta\left(e_{1}\right)= \pm e_{2}$, so that $\theta\left(e_{2}\right)= \pm e_{1}$, then we must have $\theta\left(e_{3}\right)=-e_{3}$. Hence the stabiliser of a point has order at most eight for any left invariant metric on Sol, and it is clear that if it has order eight then it is isomorphic to $D(4)$. The metric described in $\S 4$ shows that $D(4)$ can be realised. This completes the proof of Theorem 5.1.

Note that apart from $S^{3}$ and $S^{2} \times \mathbb{R}$, the total space of each of the remaining geometries is homeomorphic to $\mathbb{R}^{3}$. So it is not quite obvious from what has been said so far in this section that the eight 3-dimensional geometries are all different. Indeed in the case of the three geometries $H^{2} \times \mathbb{R}, \widetilde{\mathrm{SL}_{2} \mathbb{R}}$ and Nil it is not obvious that they are maximal. However, the following result implies at once that of the eight geometries described in $\S 4$, none is a subgeometry of any other. Hence all eight are maximal and no two are equivalent.

THEOREM 5.2. If $M$ is a closed 3-manifold which admits a geometric structure modelled on one of the eight geometries, then the geometry involved is unique.

Remark. The geometric structure on $M$ is not unique. This is the analogue of the fact that there are many flat structures on the torus and many hyperbolic structures on the closed orientable surface of genus $g, g \geqslant 2$. Neumann [42] has made some interesting connections between the space of geometric structures on $M$ and of complex structures on $M \times \mathbb{R}$. 
Proof. If $M$ admits a geometric structure modelled on $S^{3}$, the geometry involved must be unique as no other geometry has total space even homeomorphic to $S^{3}$. Similarly if $M$ admits a geometric structure modelled on $S^{2} \times \mathbb{R}$, the geometry involved must be unique.

Recall from $\$ 4$ that if $M$ admits a geometric structure modelled on one of $E^{3}$, $H^{2} \times \mathbb{R}, \widetilde{\mathrm{SL}_{2} \mathbb{R}}$ or Nil then $M$ is a Seifert fibre space. In particular, $\pi_{1}(M)$ contains a subgroup isomorphic to $\mathbb{Z} \times \mathbb{Z}$ obtained by selecting an infinite cyclic subgroup contained in the fundamental group of the base orbifold of $M$ and taking its preimage in $\pi_{1}(M)$. If $M$ admits a geometric structure modelled on Sol, then $M$ has the structure of a bundle over a 1-dimensional orbifold. As $M$ is compact the fibres are all tori or Klein bottles so that again $\pi_{1}(M)$ contains $\mathbb{Z} \times \mathbb{Z}$.

Using the above paragraph, it follows at once that if $M$ admits a geometric structure modelled on $H^{3}$, then it admits no other geometric structure. For Corollary 4.6 shows that $\pi_{1}(M)$ cannot have a subgroup isomorphic to $\mathbb{Z} \times \mathbb{Z}$.

If $M$ admits a geometric structure modelled on Sol, then $M$ has a finite covering $\tilde{M}$ which is a torus bundle over $S^{1}$ with hyperbolic glueing map. If $M$ possesses a geometric structure modelled on another geometry, we now know that this other geometry cannot be $S^{3}, S^{2} \times \mathbb{R}$ or $H^{3}$. Hence it must be one of $E^{3}, H^{2} \times \mathbb{R}, \widetilde{\mathrm{SL}_{2} \mathbb{R}}$ or Nil. Hence $M$ is a Seifert fibre space and so is $\bar{M}$. In particular, $\pi_{1}(\bar{M})$ contains an infinite cyclic normal subgroup. It is easy to show that this is impossible using the fact that a hyperbolic automorphism of $\mathbb{Z} \times \mathbb{Z}$ does not have an eigenvalue equal to 1 or -1 . We have now shown that if $M$ possesses a geometric structure modelled on $S^{3}, \quad S^{2} \times \mathbb{R}, H^{3}$ or Sol, then Theorem 5.2 holds.

To complete the proof of Theorem 5.2, we suppose that $M$ possesses geometric structures modelled on two of $E^{3}, H^{2} \times \mathbb{R}, \widehat{\mathrm{SL}_{2} \mathbb{R}}$ and $\mathrm{Nil}$ and derive a contradiction. This hypothesis implies that $M$ can be given the structure of two nonisomorphic Seifert bundles. This is because either the bundles have distinct Euler numbers or their base orbifolds have distinct Euler numbers. See Table 4.1 which summarizes the situation. Now Theorem 3.8 shows that $M$ must be covered by $S^{3}, S^{2} \times \mathbb{R}$ or $S^{1} \times S^{1} \times S^{1}$. The first two cases are impossible as our hypothesis implies that $M$ has universal covering space $\mathbb{R}^{3}$. Hence $M$ is covered by $S^{1} \times S^{1} \times S^{1}$. But our discussion in $\$ 4$ shows that $E^{3}$ is the only geometry whose isometry group has a discrete subgroup isomorphic to $\mathbb{Z} \times \mathbb{Z} \times \mathbb{Z}$. This contradiction completes the proof of Theorem 5.2.

We are now ready to give the result which classifies those closed 3-manifolds which admit a non-hyperbolic geometric structure.

THEOREM 5.3. Let $M$ be a closed 3-manifold.

(i) $M$ possesses a geometric structure modelled on Sol if and only if $M$ is finitely covered by a torus bundle over $S^{1}$ with hyperbolic glueing map. In particular, $M$ itself is either a bundle over $S^{1}$ with fibre the torus or Klein bottle or is the union of two twisted I-bundles over the torus or Klein bottle.

(ii) $M$ possesses a geometric structure modelled on one of $S^{3}, E^{3}, S^{2} \times \mathbb{R}$, $H^{2} \times \mathbb{R}, \quad \widetilde{S L}_{2}$ or Nil if and only if $M$ is a Seifert fibre space. Further if $M$ has the structure of a Seifert bundle $\eta$ over an orbifold $X$, then the appropriate geometry for $M$ 
is determined by $\chi(X)$ and $e(\eta)$ according to the following table:

$\begin{array}{llll} & \chi>0 & \chi=0 & \chi<0 \\ e=0 & S^{2} \times \mathbb{R} & E^{3} & H^{2} \times \mathbb{R} \\ e \neq 0 & S^{3} & \text { Nil } & \widetilde{S L}_{2}\end{array}$

REMARK. Each of $S^{3}, E^{3}, \widetilde{\mathrm{SL}_{2} \mathbb{R}}$, Nil and Sol is a unimodular Lie group. Let $G$ denote any one of these groups. Then many of the compact quotients of $G$ described by this theorem are obtained from the action of discrete subgroups of $G$ by left (or right) multiplication. Such quotients have been classified by Raymond and Vasquex [52].

Proof. (i) Recall from the discussion of Sol in $\$ 4$, that if $M$ admits a geometric structure modelled on Sol, then $M$ has a covering of degree at most four which is a torus bundle over $S^{1}$ with hyperbolic glueing map. Now suppose that $M$ has a finite covering $\bar{M}$ which is the total space of such a bundle. By taking a further covering, if necessary, we can assume that $\bar{M}$ is a regular covering of $M$. We have the exact sequence

$$
1 \longrightarrow \mathbb{Z} \times \mathbb{Z} \longrightarrow \pi_{1}(\bar{M}) \longrightarrow \mathbb{Z} \longrightarrow 1
$$

and it is easy to show that the $\mathbb{Z} \times \mathbb{Z}$-subgroup of $\pi_{1}(\hat{M})$ is characteristic in $\pi_{1}(\hat{M})$ and so normal in $\pi_{1}(M)$. A result of Hempel and Jaco [23] shows that $M$ itself is either a bundle over $S^{1}$ with fibre the torus or Klein bottle or is the union of two twisted $I$-bundles over the torus or Klein bottle. Now we know the structure of $M$, it is easy to see that $\pi_{1}(M)$ can act on Sol by isometries so that $M$ does possess a geometric structure modelled on Sol, as discussed in $\$ 4$.

(ii) Our discussion in $\$ 4$ has already proved half of the result stated in Theorem 5.3. It remains to prove that any Seifert fibre space admits a geometric structure. In all cases, one can do this by starting with a Seifert bundle $\eta$ over an orbifold $X$ with total space $M$ and defining an action of $\pi_{1}(M)$ on the appropriate geometry by describing the action of a set of generators. There is not much choice in how to do this, and the arguments are similar for all the geometries. In $\$ 4$, we actually proved this result directly for the geometries $S^{2} \times \mathbb{R}$ and $E^{3}$ by classifying the possible Seifert bundles. The procedure we discuss here could be used in those cases also. We will discuss first the case of $H^{2} \times \mathbb{R}$ and then $\widetilde{\mathrm{SL}}_{2}$, the other cases being handled similarly.

Let $\eta$ be a Seifert bundle over an orbifold $X$ with orientable total space $M$ and $e(\eta)=0$ and $\chi(X)<0$. We will show that $M$ admits a geometric structure modelled on $H^{2} \times \mathbb{R}$. We have the exact sequence

$$
1 \longrightarrow K \longrightarrow \pi_{1}(M) \longrightarrow \pi_{1}(X) \longrightarrow 1
$$

where $K$ is infinite cyclic. Let $k$ be a generator of $K$. We also choose a natural set of generators for $\pi_{1}(X)$ and choose elements of $\pi_{1}(M)$ which project to these generators as described in $\S 3$. Thus if $X$ is closed and orientable with $q$ cone points, one chooses generators $\bar{a}_{1}, \bar{b}_{1}, \ldots, \bar{a}_{g}, \bar{b}_{g}, \bar{x}_{1}, \ldots, \bar{x}_{q}$ of $\pi_{1}(X)$ and $\pi_{1}(X)$ has presentation

$$
\left\{\bar{a}_{1}, \bar{b}_{1}, \ldots, \bar{a}_{g}, \bar{b}_{g}, \bar{x}_{1}, \ldots, \bar{x}_{q}: \bar{x}_{i}^{\alpha_{i}}=1, \prod_{i=1}^{g}\left[\bar{a}_{i}, \bar{b}_{i}\right] \bar{x}_{1} \ldots \bar{x}_{q}=1\right\} .
$$


Let $a_{i}, b_{i}$ and $x_{i}$ be appropriate elements of $\pi_{1}(M)$, so we have the relation $x_{i}^{\alpha_{i}} k^{\beta_{i}}=1$, and $\prod\left[a_{i}, b_{i}\right] x_{1} \ldots x_{q}=k^{b}$ in $\pi_{1}(M)$. The last relation will be referred to as the long relation.

As $\chi(X)<0, \quad X$ can be regarded as the quotient of $H^{2}$ by $\pi_{1}(X)$ acting as a group of isometries. It is now clear how to define the isometries on $H^{2} \times \mathbb{R}$ which we want. First let $k$ be the product of the identity on $H^{2}$ with translation through distance 1 on $\mathbb{R}$. Let $a_{i}$ and $b_{i}$ be the product of $\bar{a}_{i}$ or $\bar{b}_{i}$ on $H^{2}$ with the identity on $\mathbb{R}$ and let $x_{i}$ be the product of $\bar{x}_{i}$ on $H^{2}$ with translation through $-\beta_{i} / \alpha_{i}$ on $\mathbb{R}$.

This defines an action of some group $G$ on $H^{2} \times \mathbb{R}$ by isometries. Clearly $k$ is central in $G$ and $G$ has all the relations of $\pi_{1}(M)$ except possibly the long relation. Now consider the element $w$ of $G$ which is the product $\prod\left[a_{i}, b_{i}\right] x_{1} \ldots x_{q}$. By construction this is an isometry of $H^{2} \times \mathbb{R}$ which projects to the identity map of $H^{2}$. Its projection onto $\mathbb{R}$ is a translation through distance $-\Sigma \beta_{i} / \alpha_{i}$. Recall the formula $e=-b-\Sigma \beta_{i} / \alpha_{i}$ connecting the Euler number $e$ of $\eta$ and the invariant $b$. As $e(\eta)$ is zero, we see that $w=k^{b}$, so that $G$ has all the relations of $\pi_{1}(M)$. It follows that $G$ is isomorphic to $\pi_{1}(M)$. For the projection Isom $\left(H^{2} \times \mathbb{R}\right) \rightarrow$ Isom $H^{2}$ when restricted to $G$ has image $\pi_{1}(X)$ and has kernel the cyclic group generated by $k$. Also $G$ acts discretely on $H^{2} \times \mathbb{R}$. Now one can see that $G$ acts freely on $H^{2} \times \mathbb{R}$. For suppose that $g$ in $G$ fixes some point of $H^{2} \times \mathbb{R}$. Then the image of $g$ in $\pi_{1}(X)$, say $\bar{g}$, must fix some point of $H^{2}$. If $\bar{g}$ is trivial, then $g$ is a power of $k$ and as $g$ fixes a point, we must have $g=1$ also. If $\bar{g}$ is non-trivial, then $\bar{g}$ must be conjugate to some power of some $\bar{x}_{i}$. Hence $g$ is conjugate to an element of the form $k^{a} x_{i}^{b}$. As $\alpha_{i}$ and $\beta_{i}$ are coprime, the group generated by $k$ and $\bar{x}_{i}$ acts freely on $H^{2} \times \mathbb{R}$. As $g$ fixes a point, we again conclude that $g$ is trivial. Hence the action of $G$ on $H^{2} \times \mathbb{R}$ has quotient which is a Seifert fibre space with base orbifold $X$ and the same Seifert invariants and the same $b$-invariant as the given Seifert bundle $\eta$. We deduce that this quotient is isomorphic to $\eta$, by the classification of Seifert bundles.

If $M$ or $X$ is non-orientable, the appropriate isometries of $H^{2} \times \mathbb{R}$ are slightly different. For example if $\bar{a}_{1}$ is a generator of $\pi_{1}(X)$ of infinite order and one wants the relation $a_{1}^{-1} k a_{1}=k^{-1}$, then one chooses $a_{1}$ to be the product of $\bar{a}_{1}$ on $H^{2}$ with a reflexion of $\mathbb{R}$. If $\bar{y}$ is an element of order two corresponding to a reflector curve in $X$, we choose $y$ to be the product of the reflexion $\bar{y}$ on $H^{2}$ with translation of $\mathbb{R}$ through distance $\frac{1}{2}$. Thus $y^{2}=k$.

This construction works equally well for the other two product geometries $S^{2} \times \mathbb{R}$ and $E^{3}$. For the twisted geometries $S^{3}, \widetilde{S L}_{2}$ and Nil one proceeds in a similar way. But more care is needed to ensure that the Euler number of the Seifert bundle constructed in this way is the same as that of the given bundle. For the product geometries, this was not a problem as the Euler number was forced to be zero.

We will discuss $\widetilde{S L}_{2}$ only, and will consider one simple example to explain how to proceed. Let $\eta$ be a Seifert bundle over the orbifold $X$ which is a 2-sphere with 3 cone points. Thus $\pi_{1}(X)$ is a triangle group $\Delta(p, q, r)$. Suppose that $\chi(X)<0$ and $e(\eta) \neq 0$. We must construct the total space $M$ of $\eta$ as a quotient of $\widetilde{S L}_{2}$ by isometries. First, we note that $X$ is the quotient of $H^{2}$ by $\Delta(p, q, r)$ and $\pi_{1}(X)=\left\{\bar{x}, \bar{y}, \bar{z}: \bar{x}^{p}=\bar{y}^{q}=\bar{z}^{r}=\bar{x} \bar{y} \bar{z}=1\right\}$. Hence we have an exact sequence

$$
1 \longrightarrow K \longrightarrow \pi_{1}(M) \longrightarrow \pi_{1}(X) \longrightarrow 1,
$$

where $K$ is infinite cyclic and so $\pi_{1}(M)$ has presentation

$\left\{k, x, y, z:[k, x]=[k, y]=[k, z]=1, x^{p} k^{\alpha}=1, y^{q} k^{\beta}=1, z^{r} k^{\gamma}=1, x y z=k^{b}\right\}$. 
We need to choose isometries $k, x, y, z$ of $\widetilde{\mathrm{SL}}_{2}$ which satisfy these relations. As before, we choose $k$ to be a non-trivial isometry of $\widetilde{S L}_{2}$ which projects to the identity map of $H^{2}$. Now there is essentially no choice for the elements $x, y, z$ as their projection to isometries of $H^{2}$ is given and we require $x^{p} k^{\alpha}=1$, etc. We must show that there is a choice of $k$ such that $x y z=k^{b}$ also, using our assumption that $e(\eta) \neq 0$. Of course, if $e(\eta)=0$, there can be no such choice.

Clearly $x y z=k^{\varepsilon}$ say, and we consider the effect of modifying our choice of $k$. Suppose we replace $k$ by $K=k^{u}$, where $u$ is some real number. Then we must replace $x$ by $X=x k^{-(u-1) x / p}$ to ensure that $X^{p} K^{\alpha}=1$, and define $Y$ and $Z$ similarly. Hence $X Y Z=k^{\prime}$, where

$$
\lambda=\varepsilon-(u-1) \alpha / p-(u-1) \beta / q-(u-1) \gamma / r .
$$

If we choose $u$ so that $\lambda=u b$, we will have the required relation $X Y Z=K^{b}$. Trivially, one can solve the equation

$$
u b=\varepsilon-(u-1) \alpha / p-(u-1) \beta / q-(u-1) \gamma / r
$$

for $u$, if and only if the coefficient of $u$ is non-zero. This coefficient is $\alpha / p+\beta / q+\gamma / r+b$ which is exactly $-e(\eta)$ and so is non-zero by hypothesis.

A similar discussion will apply to more general Seifert fibre spaces, including non-compact ones.

At this point, we see that the classification of Seifert fibre spaces gives a complete classification of those closed 3-manifolds which possess a geometric structure modelled on $S^{3}, E^{3}, \quad S^{2} \times \mathbb{R}, H^{2} \times \mathbb{R}, \quad \widetilde{S L}_{2}$ or Nil. We also have a description of those closed 3-manifolds which admit a geometric structure modelled on Sol. These manifolds can be classified but the answer is somewhat complicated. For example, consider the collection of all torus bundles over $S^{1}$ with hyperbolic glueing map. The action of the glueing map on $H_{1}\left(S^{1} \times S^{1}\right)$ determines a matrix in $\mathrm{GL}_{2}(\mathbb{Z})$, and the total spaces of these bundles are classified by the conjugacy class in $G_{2}(\mathbb{Z})$ of this matrix. Dunbar has announced the classification of all 3-dimensional orbifolds which admit a geometric structure modelled on Sol.

We saw in $\$ 4$ that a closed manifold $M$ with a geometric structure modelled on $E^{3}, S^{2} \times \mathbb{R}$ or $H^{2} \times \mathbb{R}$ naturally inherits the structure of a surface bundle over a 1 -dimensional orbifold and this structure is dual to the Seifert fibre structure on $M$. In particular, if the base of this bundle is a circle, the bundle has periodic glueing map. The following result summarises this situation.

THEOREM 5.4. (i) If $M^{3}$ is a surface bundle over $S^{1}$ with periodic glueing map, then $M$ has the structure of a Seifert bundle $\eta$ with $e(\eta)=0$.

(ii) If $M^{3}$ is the total space of a Seifert bundle $\eta$ with $e(\eta)=0$ and if the cyclic subgroup of $\pi_{1}(M)$ carried by a regular fibre is central, then $M$ is a surface bundle over $S^{1}$ with periodic glueing map.

REMARK. A common version of (ii) takes as hypothesis that the cyclic subgroup of $\pi_{1}(M)$ carried by a regular fibre injects into $H_{1}(M)$. See $[48,47]$. This is equivalent to the hypothesis in (ii). 
Proof. Statement (i) is clear, as discussed at the start of $\$ 4$.

(ii) As $e(\eta)=0$, Theorem 5.3 shows that $M$ admits a geometric structure modelled on one of the product geometries $E^{2} \times \mathbb{R}, S^{2} \times \mathbb{R}$ or $H^{2} \times \mathbb{R}$. Hence, as discussed in $\S 4, M$ admits the structure of a surface bundle over a 1-dimensional orbifold $Y$. This orbifold is obtained as a quotient of $\mathbb{R}$ by projecting the action of $\pi_{1}(M)$ on $E^{2} \times \mathbb{R}, S^{2} \times \mathbb{R}$ or $H^{2} \times \mathbb{R}$ onto the $\mathbb{R}$-factor. The cyclic subgroup $K$ of $\pi_{1}(M)$ carried by a regular fibre acts by translation on this $\mathbb{R}$-factor. The hypothesis that $K$ is central implies that no reflexions of the $\mathbb{R}$-factor occur. Hence $Y$ must be a circle. Hence $M$ has the structure of a surface bundle over $S^{1}$ with periodic glueing map.

We have now met torus bundles over $S^{1}$ in the context of three different geometries-namely $E^{3}$, Nil and Sol. The following result explains and summarises the situation. Recall that a matrix in $\mathrm{GL}_{2}(\mathbb{Z})$ is hyperbolic if neither of its eigenvalues has absolute value 1 .

THEOREM 5.5. Let $M^{3}$ be the total space of a torus bundle over $S^{1}$ with glueing map $\phi$, and let $A$ in $\mathrm{GL}_{2}(\mathbb{Z})$ represent the automorphism of $H_{1}\left(S^{1} \times S^{1}\right)$ induced by $\phi$.

(i) If $\mid$ trace $A \mid>2$, then $A$ is hyperbolic and $M$ admits a Sol-structure.

(ii) If $A$ is periodic, then $M$ admits a $E^{3}$-structure.

(iii) If $\mid$ trace $A \mid \leqslant 2$, then either $A$ is periodic, so that $M$ admits a $E^{3}$-structure, or $A$ is not periodic and $M$ admits a Nil-structure, or $A$ is hyperbolic and $M$ admits a Sol-structure.

Proof. Let $A=\left(\begin{array}{ll}a & b \\ c & d\end{array}\right)$, where $a d-b c= \pm 1$. Then the characteristic
polynomial of $A$ is

$$
\lambda^{2}-(a+d) \lambda+(a d-b c)=0 .
$$

(i) If $|a+d|>2$, this polynomial has real eigenvalues which cannot be 1 or -1 , so $A$ is hyperbolic.

Now one can show that $M$ admits a Sol-structure as discussed in Theorem 5.3 and in $\S 4$.

(ii) This case follows from Theorem 5.4 (i), and the fact that if $A$ is periodic, then $\phi$ is isotopic to a periodic diffeomorphism of the torus.

(iii) If $|a+d|<2$, then $a+d$ equals $-1,0$ or 1 . This implies that the eigenvalues of $A$ are distinct complex numbers and are roots of unity. It follows that $A$ is periodic so that $M$ admits a $E^{3}$-structure by part (ii).

If $|a+d|=2$ and $a d-b c=-1$, then the eigenvalues of $A$ do not have absolute value 1 . Hence $A$ is hyperbolic and $M$ admits a Sol-structure.

If $|a+d|=2$ and $a d-b c=1$, then the eigenvalues of $A$ are equal and their common value is 1 or -1 . Hence $A$ is conjugate in $\mathrm{GL}_{2}(\mathbb{R})$ to one of $\left(\begin{array}{ll}1 & 0 \\ 0 & 1\end{array}\right)$, $\left(\begin{array}{rr}-1 & 0 \\ 0 & -1\end{array}\right),\left(\begin{array}{ll}1 & 1 \\ 0 & 1\end{array}\right)$ or $\left(\begin{array}{rr}-1 & 1 \\ 0 & -1\end{array}\right)$. If $A$ is not periodic, the only possible case is 
$\left(\begin{array}{ll}1 & 1 \\ 0 & 1\end{array}\right)$, so that both eigenvalues of $A$ are equal to 1 and $A$ has a unique eigenvector. Now it follows that $A$ is conjugate in $\mathrm{GL}_{2}(\mathbb{Z}) \overline{\text { to }}\left(\begin{array}{ll}1 & n \\ 0 & 1\end{array}\right)$ for some integer $n$. Hence we know that $M$ must admit a Nil-structure from the discussion of Nil in $\S 4$.

\section{§6. The Geometrisation Conjecture}

I want to close this article by briefly discussing the present position on Thurston's Geometrisation Coniecture. This conjecture asserts that any compact 3-manifold can be cut in a reasonably canonical way into a union of geometric pieces. In fact, the decomposition does exist. The point of the conjecture is that the pieces should all be geometric.

We write $M_{1} \# M_{2}$ to denote the connected sum of $M_{1}$ and $M_{2}$. Note that $M \# S^{3}$ is always homeomorphic to $M$. A 3-manifold $M$ is called prime if any expression of $M$ as $M_{1} \# M_{2}$ has $M_{1}$ or $M_{2}$ homeomorphic to $S^{3}$. A theorem of Kneser [28] asserts that any compact 3-manifold can be expressed as a finite connected sum of primes, and Milnor [37] showed that the factors involved are unique if $M$ is orientable. If $M$ is non-orientable, one can describe precisely the ambiguity which arises. It is caused by the fact that if $N$ is non-orientable then $N \#\left(S^{2} \times S^{1}\right)$ and $N \#\left(S^{2} \tilde{x} S^{1}\right)$ are homeomorphic. However, the family of spheres defining this decomposition of $M$ into primes is not unique up to isotopy even when $M$ is orientable.

So far, we have seen that a compact 3-manifold can be split in a reasonably canonical way into prime 3-manifolds. Note that if $M=M_{1} \# M_{2}$, then $M_{1}$ and $M_{2}$ are not submanifolds of $M$. They are obtained by cutting $M$ along a separating 2-sphere and then adding a 3-ball to each of the manifolds obtained.

Next we need to consider compact, orientable, prime 3-manifolds. I will discuss the non-orientable case later. There are three basic types of these manifolds. Firstly those with finite fundamental group. Such a manifold is either a 3-ball or is closed and is covered by a homotopy sphere [21]. For these manifolds, Thurston's Conjecture can be stated as follows.

CONJECture. If $M^{3}$ is closed and orientable and $\pi_{1}(M)$ is finite, then $M$ possesses a geometric structure modelled on $S^{3}$.

This conjecture asserts that the Poincare Conjecture is true and that all free actions of finite groups on $S^{3}$ are conjugate to orthogonal actions. Some progress has been made on the second part of the conjecture, but on the other hand it is still not proved even when the group is $\mathbb{Z}_{3}$. Milnor [36] listed the groups which might act freely on $S^{3}$. These included the subgroups of $S O(4)$ which were described in $\$ 4$, and some other families of groups. Some of the groups on Milnor's list which are not subgroups of $S O(4)$ were proved not to act freely on $S^{3}$ by Lee [31], and the remainder were eliminated by Thomas [65] on the assumption that the Smale Conjecture is true. Hatcher [19] has now proved the Smale Conjecture. Thus it is now known that if a finite group $G$ acts freely on $S^{3}$, then $G$ is isomorphic to a subgroup of SO (4). It is known that if $G$ is of order $2^{k}$ or is non-cyclic of order $2^{l} \cdot 3$, where $l \geqslant 1$, then all free actions of $G$ on $S^{3}$ are conjugate to orthogonal actions. This result is also known if $G$ is non-cyclic, non-dihedral and has order $2^{k} 3^{m}$ (see 
[56]). See [41] for a discussion of the connections between these results. See also [12, $32,53,54$ and 55].

The compact, orientable prime 3-manifolds with infinite fundamental group fall into two types according to whether or not they admit a non-separating embedded sphere. Note that the hypothesis of primeness implies that any separating sphere must bound a ball. It is not hard to show that $S^{2} \times S^{1}$ is the only orientable prime manifold with a non-separating embedded sphere [21]. All the other compact, orientable prime 3-manifolds are irreducible, i.e. every embedded 2-sphere bounds a ball. Now the Sphere Theorem implies that if $M$ is orientable and irreducible then $\pi_{2}(M)=0$. If, in addition, $\pi_{1}(M)$ is infinite, then it is a standard result that the universal covering of $M$ is contractible so that $M$ itself is aspherical.

Now consider a compact, orientable, irreducible 3-manifold $M$ with infinite fundamental group. It may or may not have boundary. If $\partial M$ is compressible in $M$, then the Loop Theorem gives a 2-disc $D$ embedded in $M$ so that $D \cap \partial M$ is an essential curve on $\partial M$. In this case, one cuts $M$ along $D$ to obtain a new 3-manifold $N$. Clearly $M$ can be obtained from $N$ by attaching a 1 -handle to $\partial N$. If $\partial N$ is compressible in $N$, one cuts $N$ along another disc. This process must stop and yields a splitting of $M$ into pieces each of which has incompressible boundary. The pieces which are not 3-balls are uniquely determined up to isotopy in $M$, but the 2-discs which split $M$ are not unique up to isotopy. For example, if $M$ is a handle body of genus 2 , it can be split in infinitely many ways by two non-separating discs.

We have now reduced to the case of a compact, orientable, irreducible 3-manifold $M$ with incompressible boundary. (This includes the case when $\partial M$ is empty.) Now Johannson [26] and Jaco and Shalen [25] have shown that there is a finite family of disjoint, incompressible, 2-sided, embedded tori in $M$ which splits $M$ into pieces which are either Seifert fibre spaces or admit no embedded incompressible torus except possibly parallel to the boundary. (A few manifolds satisfy both these conditions.) Further, they show that a minimal such family of tori in $M$ is unique up to isotopy. Of course, this family may be empty, for example, if $M$ is a Seifert fibre space. It is the pieces of this last decomposition which are conjectured to admit geometric structures. We already know that Seifert fibre spaces admit geometric structures, so we need only consider the case of a compact, orientable, irreducible 3-manifold $M$ in which every embedded, incompressible torus is parallel to $\partial M$. If $M$ is Haken and is not a Seifert fibre space, then the Torus Theorem $[14,15,25,26,35$, 57] shows that any subgroup of $\pi_{1}(M)$ isomorphic to $\mathbb{Z} \times \mathbb{Z}$ is conjugate into the fundamental group of some component of $\partial M$. A manifold satisfying this condition is called atoroidal. Now Thurston has proved that such a manifold possesses a hyperbolic structure. See [66-70]. It follows that Thurston's Geometrisation Conjecture holds for all Haken manifolds, and hence, of course, all connected sums of Haken manifolds.

Note that if $M$ is a torus bundle over $S^{1}$ with hyperbolic glueing map and so has a geometric structure modelled on Sol, then the above splitting procedure would cut $M$ along a fibre torus into $N=S^{1} \times S^{1} \times I$. Of course, $N$ does possess a geometric structure, but clearly, in this case, it would be better not to split $M$ at all. A similar point occurs when one considers the connected sum splitting. For $P^{3} \# P^{3}$ admits a geometric structure modelled on $S^{2} \times \mathbb{R}$, but one can also split this manifold into two copies of $P^{3}$ each of which possesses a geometric structure modelled on $S^{3}$.

We are left with the non-Haken orientable irreducible 3-manifolds with infinite fundamental group to consider. All such manifolds must be closed. Many examples 
of these are known now, but all are Seifert fibre spaces or admit a hyperbolic structure and the Geometrisation Conjecture asserts that this is always the case. Precisely, one has the following:

Conjecture. Let $M^{3}$ be closed, orientable and irreducible with infinite fundamental group but not Haken. Then $M$ is a Seifert fibre space or admits a hyperbolic structure.

Very little progress has been made on this conjecture. There is a related conjecture which asserts that if $M$ satisfies these conditions then there is a finite covering $M_{1}$ of $M$ which is Haken. See [22]. If one knew this to be the case, it would follow that $M_{1}$ possessed a geometric structure. For otherwise the canonical family of tori in $M_{1}$ is non-empty and it can be shown that this family can be chosen to be invariant under the covering group. Thus $M$ would contain an incompressible torus and so be Haken. If $M_{1}$ is a Seifert fibre space, recent work of the author [58] (see also [4]) shows that $M$ also is a Seifert fibre space and so possesses a geometric structure. If $M_{1}$ is hyperbolic, then $\pi_{1}(M)$ is a torsion free finite extension of $\pi_{1}\left(M_{1}\right)$, and $\pi_{1}\left(M_{1}\right)$ acts on $H^{3}$ as a group of isometries with compact quotient. Now the Mostow Rigidity Theorem [40] implies that there is an action of $\pi_{1}(M)$ on $H^{3}$ by isometries which extends the action of $\pi_{1}\left(M_{1}\right)$. Thus $M_{1}$ covers a hyperbolic manifold $N$ with $\pi_{1}(N)$ isomorphic to $\pi_{1}(M)$. Hence $M$ is homotopy equivalent to a hyperbolic manifold. However, it is not known whether this implies that $M$ and $N$ are homeomorphic - not even when $M$ is only double covered by $M_{1}$.

If one considers a non-Haken 3-manifold $M$ with infinite fundamental group and does not assume that $M$ is finitely covered by a Haken manifold, then essentially nothing at all can be said about $M$. For example the following conjectural characterisation of Seifert fibre spaces is implied by the Geometrisation Conjecture but is unknown unless $M$ is covered by a Haken manifold $[72,73,18,25,26,49]$.

CONJECTURE. Let $M$ be a compact, orientable, irreducible 3-manifold with infinite fundamental group. Then $M$ is a Seifert fibre space if and only if $\pi_{1}(M)$ contains an infinite cyclic normal subgroup.

This conjecture alone is very interesting. If proved, it would give the following conjectural characterisation of Fuchsian groups.

CONJECTURE. A finitely generated group $\Gamma$ is isomorphic to a Fuchsian group, that is a discrete subgroup of $\operatorname{PSL}(2, \mathbb{R})$, if and only if there is an action of $\Gamma$ on $S^{1}$ by homeomorphisms such that the action of $\Gamma$ on $S^{1} \times S^{1} \times S^{1}$, in which $\Gamma$ acts on each component separately, becomes a covering action when restricted to $S^{1} \times S^{1} \times S^{1}-\Delta$, where $\Delta=\left\{(x, y, z) \in S^{1} \times S^{1} \times S^{1}:\right.$ two of $x, y, z$ are equal $\}$.

The connection between these two conjectures is simply that $S^{1} \times S^{1} \times S^{1}-\triangle$ is homeomorphic to $S^{1} \times \mathbb{R}^{2}$. Hence its quotient by $\Gamma$ is a 3 -manifold $M$ such that one has an exact sequence

$$
1 \longrightarrow \mathbb{Z} \longrightarrow \pi_{1}(M) \longrightarrow \Gamma \longrightarrow 1 .
$$

Now I want to consider compact, non-orientable, prime 3-manifolds. If such a manifold $M$ contains a non-separating 2 -sphere, it must be homeomorphic to $S^{2} \tilde{\times} S^{1}$ 
[21]. Otherwise $M$ is irreducible. If $M$ does not contain a 2-sided projective plane, then $\pi_{2}(M)=0$, by the Projective Plane Theorem [9]. Now $\pi_{1}(M)$ cannot be finite in this case, so it follows that $M$ is aspherical. Further it is easy to show that $M$ must be Haken. Hence, Thurston's work on hyperbolic structures shows that the Geometrisation Conjecture holds for $M$. Note that one finds a canonical family of tori and Klein bottles splitting $M$ instead of just tori as in the orientable case.

If $M$ is compact, non-orientable and irreducible, but contains 2-sided projective planes, there is a splitting of $M$ by disjoint 2-sided projective planes into pieces for each of which any 2-sided $P^{2}$ is parallel to a boundary component. Swarup [64] has considered such splittings. Assume now that $M$ itself has the property that any 2 -sided $P^{2}$ in $M$ is parallel to a boundary component of $M$. Note that no component of $\partial M$ can be a sphere. If $\pi_{1}(M)$ is finite, Epstein [9] showed that it must have order two and that $M$ is homotopy equivalent to $P^{2} \times I$. If the Poincare Conjecture holds then $M$ must be homeomorphic to $P^{2} \times I$. Of course, $P^{2} \times I$ possesses a geometric structure modelled on $S^{2} \times \mathbb{R}$. (Recall that the geometric structure is really on the interior of the manifold.) If $\pi_{1}(M)$ is infinite, we start by asking if $\partial M$ is compressible. If $\partial M$ is compressible, we cut $M$ along 2-discs until we reach a manifold with incompressible boundary.

Let $M$ be a compact, non-orientable, irreducible 3-manifold with incompressible boundary and suppose that any 2-sided projective plane in $M$ is parallel to a boundary component. Let $M_{1}$ be the orientable double covering of $M$ and let $\bar{M}_{1}$ denote the union of $M_{1}$ with a 3-ball attached to each boundary sphere of $M_{1}$. Then $\bar{M}_{1}$ is compact, orientable and irreducible and has incompressible boundary. Now Johannson [26] and Jaco and Shalen [25] tell us that $\bar{M}_{1}$ has a family of disjoint tori which is unique up to isotopy. This family can be chosen so as to lie in $M_{1}$ and be invariant under the covering translation. Hence $M$ itself contains a canonical family of disjoint tori and Klein bottles. If we split $\bar{M}_{1}$ along its family of tori, the conjecture is that each piece has a geometric structure. If we split $M$ along its corresponding family of tori and Klein bottles, any piece with a projective plane boundary component cannot admit a geometric structure. For the only compact 3-manifold which has a boundary $P^{2}$ and admits a geometric structure is $P^{2} \times I$. This is because $S^{2} \times \mathbb{R}$ is the only geometry whose total space has non-zero $\pi_{2}$. The Geometrisation Conjecture in this case needs to be modified slightly. Let $X$ denote the cone on $P^{2}$ and attach a copy of $X$ to each boundary $P^{2}$ of $M$ to obtain $\bar{M}$. Regard $X$ as the orbifold $D^{3} / \mathbb{Z}_{2}$, where $\mathbb{Z}_{2}$ acts on $D^{3}$ by the map $\mathbf{x} \rightarrow-\mathbf{x}$, so that $\bar{M}$ is an orbifold. In fact $\bar{M}=\bar{M}_{1} \mid \mathbb{Z}_{2}$. It is conjectured that each piece of $\bar{M}$, regarded as an orbifold, admits a geometric structure. This is equivalent to asserting that each piece of $\bar{M}_{1}$ admits a geometric structure and that the involution on it preserves this structure.

\section{References}

1. J. W. Alexander, 'On the subdivision of 3-space by a polyhedron', Proc. Nat. Acad. Sci. U.S.A., 10 (1924), 6-8.

2. L. Auslander, L. Green and F. Hahn, Flow on homogeneous spaces, Ann. of Math. Studies 53 (Princeton University Press, Princeton, N.J., 1963).

3. L. BiEbERBACH, 'Ueber die Bewegungsgruppen der Euklidischen Räume I', Math. Ann., 70 (1911), 297-336.

4. H. BOEHME, 'Fast genugend grosse irreduzible 3-dimensionale Mannigfaltigkeiten', Invent. Math., 17 (1972), 303-316.

5. F. Bonahon and L. Siebenmann, 'New geometrical splittings of classical knots and links', preprint, Orsay University, Paris. 
6. S. BundgaArd and J. Nielsen, 'On normal subgroups with finite index in F-groups', Mat. Tidsskr. B, (1951), 56-58.

7. W. DunbaR, 'Fibered orbifolds and crystallographic groups', Thesis, Princeton University, 1981.

8. B. ECKMANN and H. MULLer, 'Plane motion groups and virtual Poincare duality of dimension two', Invent. Math., 69 (1982), 293-310.

9. D. B. A. EPSTEIN, 'Projective planes in 3-manifolds', Proc. London Math. Soc., 11 (1961), 469-484.

10. D. B. A. EPSTEIN, 'Curves on 2-manifolds and isotopies', Acta Math., 115 (1966), 83-107.

11. D. B. A. EpStein, 'Periodic flows on 3-manifolds', Ann. of Math., 95 (1972), 66-82.

12. B. Evans and J. Maxwell, 'Quaternion actions on $S^{3}$, Amer. J. Math., 101 (1979), 1123-1130.

13. W. FENCHEL, 'Estensioni di gruppi discontinui e transformazioni periodiche delle superficie', Atti Accad. Naz. Lincei Rend. Cl. Sci. Fis. Mat. Natur. (8), 5 (1948), 326-329.

14. C. D. Feustel, 'On the torus theorem and its applications', Trans. Amer. Math. Soc., 217 (1976), 1-43.

15. C. D. Feustel, 'On the torus theorem for closed 3-manifolds', Trans. Amer. Math. Soc., 217 (1976), 45-57.

16. R. FintUSHEL, 'Local $S^{1}$-actions on 3-manifolds', Pacific J. Math., 66 (1976), 111-118.

17. R. H. Fox, 'On Fenchel's Conjecture about $F$-groups', Mat. Tidsskr. B (1952), 61-65.

18. C. GORDON and W. HEIL, 'Cyclic normal subgroups of fundamental groups of 3-manifolds', Topology, 14 (1975), 305-309.

19. A. Hatcher, 'A proof of the Smale Conjecture, Diff $\left(S^{3}\right) \simeq O(4)$ ', Ann. of Math., 117 (1983), 553-607. (1979), 220.

20. W. Hell, 'On $P^{2}$-irreducible 3-manifolds', Bull. Amer. Math. Soc., 75 (1963), 772- 775.

21. J. HeMPEL, 3-manifolds, Ann. of Math. Studies 86 (Princeton University Press, Princeton, N.J., 1976.

22. J. HEMPEL, 'Orientation reversing involutions and the first Betti number for finite coverings of 3-manifolds', Invent. Math., 67 (1982), 133-142.

23. J. HeMPEL and W. JACO, 'Fundamental groups of 3-manifolds which are extensions', Ann. of Math., 95 (1972), 86-98.

24. H. HOPF, 'Zum Clifford-Kleinschen Raumproblem', Math. Ann., 95 (1925-26), 313-319.

25. W. JACO and P. B. Shalen, 'Seifert fibred spaces in 3-manifolds', Mem. Amer. Math. Soc., (1979), 220.

26. K. Johannson, Homotopy equivalences of 3-manifolds with boundary, Lecture Notes in Mathematics 761 (Springer, Berlin, 1979).

27. S. P. KerCKhoff. 'The Nielsen Realisation Problem', Ann. of Math., 117 (1983), 235-265.

28. H. KNESER, 'Geschlossene Flächen in dreidimensionale Mannigfaltigkeiten', Jahresber. Deutsch. Math.-Verein., 38 (1929), 248-260.

29. R. S. KulkarNi and F. RaYmond, 'Three dimensional Lorentz space forms and Seifert fibre spaces', preprint, University of Michigan, Ann Arbor, Michigan 48109.

30. F. LaUdenbach, 'Topologie de la dimension trois homotopie et isotopie', Asterisque, 12 (1974).

31. R. LeE, 'Semicharacteristic classes', Topology, 12 (1973), 183-200.

32. G. R. Livesay, 'Fixed point free involutions on the 3-sphere', Ann. of Math., 72 (1960), 603-611.

33. A. M. MACBEATH, 'On a theorem by J. Nielsen', Quart. J. Math., 13 (1962), 235-236.

34. A. M. MACBEATH, 'The classification of non-euclidean plane crystallographic groups', Canad. J. Math., 6 (1967), 1192-1205.

35. R. T. MiLLER, 'A new proof of the homotopy torus and annulus theorems', preprint, Michigan State University, East Lansing, Michigan 48824.

36. J. MILNOR, 'Groups which act on $S^{n}$ without fixed points', Amer. J. Math., 79 (1957), 623-630.

37. J. MILNOR, 'A unique factorisation theorem for 3-manifolds', Amer. J. Math., 84 (1962), 1-7.

38. J. MiLnOR, 'Curvatures of left invariant metrics on Lie groups', Adv. in Math., 21 (1976), 293-329.

39. J. MILNOR, 'Hyperbolic geometry: the first 150 years', Bull. Amer. Math. Soc., 6 (1982), 9-24.

40. G. D. Mostow, Strong rigidity of locally symmetric spaces, Ann. of Math. Studies 78 (Princeton University Press, Princeton, N.J., 1973).

41. R. MYERS, 'Free involutions on lens spaces', Topology, 20 (1981), 313-318.

42. W. D. NeumanN, 'Geometry of quasi-homogeneous surface singularities', Proc. Sympos. Pure Math., 40, to appear.

43. W. D. NeumanN, 'Seifert manifolds', lecture notes available from Brandeis University (1981).

44. W. D. NeumanN and F. Raymond, 'Seifert manifolds, plumbing, $\mu$-invariant and orientation reversing maps', Lecture Notes in Mathematics 664 (Springer, Berlin, 1978), pp. 162-195.

45. J. NielSEN, 'Abbildungsklassen endlicher Ordnung', Acta Math., 75 (1943), 23-115.

46. P. OrLIK, Seifert manifolds, Lecture Notes in Mathematics 291 (Springer, Berlin, 1972).

47. P. ORLIK and F. RAYMOND, 'On 3-manifolds with local SO (2) action', Quart. J. Math. Oxford, 20 (1969), $143-160$.

48. P. ORLIK, E. VoGt and H.ZIESChANG, 'Zur Topologie gefaserter dreidimensionaler Mannigfaltigkeiten', Topology, 6 (1967), 49-64.

49. N. PelczYnski and H. Zieschang, 'On coverings of Seifert 3-manifolds', Arch. Math. (Basel), 31 (1978-79), 382-386.

50. G. Polya, 'Ueber die Analogie der Kristallsymmetrie in der Ebene', Z. Kristall., 60 (1924), 278-282. 
51. F. Raymond, 'Classification of the actions of the circle on 3-manifolds', Trans. Amer. Math. Soc., 131 (1968), 51-78.

52. F. RAYMOND and A. T. VASQUEZ, '3-manifolds whose universal coverings are Lie groups', Topology Appl., 12 (1981), 161-179.

53. P. RICE, 'Free actions of $\mathbb{Z}_{4}$ on $S^{3}$, Duke Math. J., 36 (1969), 749-751.

54. G. RITTER, 'Free actions of $\mathbb{Z}_{8}$ ', Trans. Amer. Math. Soc., 181 (1973), 195-212.

55. J. H. RUBINSTEIN, 'On 3-manifolds that have finite fundamental group and contain Klein bottles', Trans. Amer. Math. Soc., 251 (1979), 129-137.

56. J. H. RUBinstein, 'Free actions of some finite groups on $S^{3}$,' Math. Ann., 240 (1979), 165-175.

57. P. SCOTT, 'A new proof of the annulus and torus theorems', Amer. J. Math., 102 (1980), 241-277.

58. P. ScotT, 'There are no fake Seifert fibre spaces with infinite $\pi_{1}$ ', Ann. of Math., 117 (1983), 35-70.

59. H. SEIFERT, 'Topologie dreidimensionaler gefaserter Räume', Acta Math., 60 (1933), 147-238.

60. H. Seifert and W. Threlfall, A textbook of topology, Pure and Applied Mathematics 89 (Academic Press, 1980).

61. A. Selberg, 'On discontinuous groups in higher dimensional symmetric spaces', Contributions to function theory (Bombay, 1960), pp. 147-164.

62. L. SiebenmanN, 'Introduction aux espaces fibrés de Seifert', notes at Orsay University.

63. I. M. Singer, 'Infinitesimally homogeneous spaces', Comm. Pure Appl. Math., 13 (1960), 685-697.

64. G. A. Swarup, 'Projective planes in irreducible 3-manifolds', Math. Z., 132 (1973), 305-317.

65. C. B. ThомAS, 'Free actions by finite groups on $S^{3}$ ', Proc. Sympos. Pure Math. No. 32, Part 1 (American Mathematics Society, Providence, R.I., 1978), pp. 125-130.

66. W. P. ThuRston, The geometry and topology of 3-manifolds, to be published by Princeton University Press.

67. W. P. ThuRston, 'Hyperbolic geometry and 3-manifolds', Low-dimensional topology, London Mathematical Society Lecture Note Series 48 (ed. Brown and Thickstun, Cambridge University Press, 1982), pp. 9-25.

68. W. P. ThuRston, 'Three dimensional manifolds, Kleinian groups and hyperbolic geometry', Bull. Amer. Math. Soc., 6 (1982), 357-381.

69. W. P. ThuRston, 'Hyperbolic structures on 3-manifolds, I: deformations of acylindrical manifolds', to appear in Annals of Mathematics.

70. W. P. ThURSTON, 'Hyperbolic structures on 3-manifolds, II: surface groups and 3-manifolds which fibre over the circle', preprint, Princeton University.

71. J. Tollefson, 'The compact 3-manifolds covered by $S^{2} \times \mathbb{R}$ ', Proc. Amer. Math. Soc., 45 (1974), 461-462.

72. F. WALDhAUSEN, 'Eine Klasse von 3-dimensionalen Mannigfaltigkeiten I', 'Il', Invent. Math., 3 (1967), 308-333 and ibid., 4 (1967), 501-504.

73. F. WALDHAUSEN, 'Gruppen mit Zentrum und 3-dimensionale Mannigfaltigkeiten', Topology, 6 (1967), 505-517.

74. F. WALDHAUSEN, 'On irreducible 3-manifolds which are sufficiently large', Ann. of Math., 87 (1968), 56-88.

75. J. A. WOLF, Spaces of constant curvature (McGraw-Hill, New York, 1967).

76. H. ZIESCHANG, Surfaces and planar discontinuous groups, Lecture Notes in Mathematics 835 (Springer, Berlin, 1980).

77. H. ZIESCHANG, Finite groups of mapping classes of surfaces, Lecture Notes in Mathematics 875 (Springer, Berlin, 1980).

78. H. ZIESCHANG, E. VOGT and H.-D. COLDEWEY, Flächen und ebene diskontinuerliche Gruppen, Lecture Notes in Mathematics 122 (Springer, Berlin, 1970).

79. B. ZIMMERMAN, 'Endliche Erweiterungen nichteuklidischer kristallographischer Gruppen', Math. Ann., $231(1977), 187-192$.

\section{[Additional references received after acceptance of paper]}

80. R. S. Kulkarni, 'Proper actions and pseudo-Riemannian space-forms', Adv. in Math., 40 (1981), 10-51.

81. R. S. KUlKarni, 'Fundamental groups of homogeneous space forms', Math. Ann., 234 (1978), 51-60.

82. M. P. RyAN and L. C. ShePley, Homogeneous relativistic cosmologies, Princeton Series in Physics (Princeton University Press, Princeton, N.J., 1975).

Department of Mathematics, University of Michigan, Ann Arbor, Michigan 48109, U.S.A.
Permanent address:

Department of Pure Mathematics, University of Liverpool, P.O. Box 147, Liverpool L69 3BX. 